\title{
Bigraded invariants for real curves
}

\author{
PEDRO F DOS SANTOS \\ PAULO LIMA-FILHO
}

\begin{abstract}
For a proper smooth real algebraic curve $\Sigma$ we compute the ring structure of both its ordinary bigraded $\operatorname{Gal}(\mathbb{C} / \mathbb{R})$-equivariant cohomology [6] and its integral Deligne cohomology for real varieties [12]. These rings reflect both the equivariant topology and the real algebraic structure of $\Sigma$ and they are recipients of natural transformations from motivic cohomology. We conjecture that they completely detect the motivic torsion classes.
\end{abstract}

$55 \mathrm{~N} 91 ; 14 \mathrm{P} 25$

\section{Introduction}

Curves are basic objects in algebraic geometry, yet their study provides a first insight into the subtleties of algebraic varieties. Over nonalgebraically closed fields $k$ this complexity is further augmented by the existence of distinct varieties that become isomorphic to the same variety $X$ after extending scalars to the algebraic closure $\bar{k}$ of $k$, the various $k$-forms of $X$.

Over the real numbers $k=\mathbb{R}$ the set of complex points of a proper smooth algebraic curve $\Sigma$ becomes a compact Riemann surface $\Sigma(\mathbb{C})$ when endowed with the analytic topology. It comes equipped with an antiholomorphic involution $\sigma: \Sigma(\mathbb{C}) \rightarrow \Sigma(\mathbb{C})$ induced by the action of $\mathfrak{S}=\operatorname{Gal}(\mathbb{C} / \mathbb{R})$ whose fixed points are the real points $\Sigma(\mathbb{R})$. The existence of connections between the topological invariants of the $\mathfrak{S}$-space $\Sigma(\mathbb{C})$ and the algebraic invariants of $\Sigma$ is well known, reflects the differences between the various $\mathbb{R}$-forms of $\Sigma_{\mathbb{C}}$, and has been explored in important references such as Gross and Harris [5] and Pedrini and Weibel [8], among others.

In this paper we study two sets of bigraded cohomological invariants that detect both the topological $\mathfrak{S}$-space structure of $\Sigma(\mathbb{C})$ and the algebraic structure of $\Sigma$ as a real variety.

The most basic topological invariant is the type of the curve: $\Sigma$ is said to be of separating type if $\Sigma(\mathbb{C}) \backslash \Sigma(\mathbb{R})$ is disconnected. Otherwise $\Sigma$ is of nonseparating type. If $\Sigma$ has genus $g$ and the number of connected components of $\Sigma(\mathbb{R})$ is $r$, we 
say that $\Sigma$ has topological type $\Sigma_{g, r}^{\varepsilon}$, where $\varepsilon=1$ if $\Sigma$ is separating and $\varepsilon=0$ if $\Sigma$ is nonseparating.

It turns out (see Silhol [14, Section 1]) that the equivariant homeomorphism type of $\Sigma(\mathbb{C})$ is completely determined by the invariants $(g, r, \varepsilon)$, which are subject to the following restrictions:

- If $\varepsilon=1$ then $1 \leq r \leq g+1$ and $g \equiv r+1 \bmod 2$.

- If $\varepsilon=0$ then $0 \leq r \leq g$.

In $\mathfrak{S}$-equivariant algebraic topology, the counterpart of singular cohomology is ordinary $\mathrm{RO}(\mathfrak{S})$-graded equivariant cohomology (see Lewis, May and McClure [6]) which is a bigraded ring $H_{\mathrm{Br}}^{*, \bullet}(\Sigma(\mathbb{C}) ; \underline{\mathbb{Z}})$. This is the first of the bigraded invariants of $\Sigma$ that we study by computing its ring structure in Section 4. It is worth mentioning that there are still very few examples of complete calculations of these bigraded equivariant cohomology rings (see Lewis [7], the authors [11] and Dugger [3]). To illustrate our calculations, let us consider the case $\Sigma=\Sigma_{g, g+1}^{1}$, depicted in Figure 1.

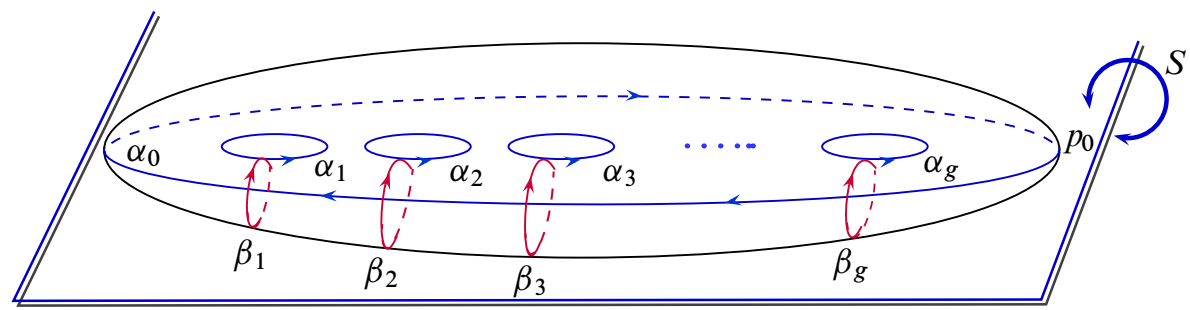

Figure 1: Curve of separating type $\Sigma_{g, g+1}^{1}$

As a module over $\mathcal{B}$, the cohomology ring of a point, we have

$$
H_{\mathrm{Br}}^{*, \bullet}(\Sigma(\mathbb{C}) ; \underline{\mathbb{Z}}) \cong \mathcal{B} \cdot \mathbf{1} \oplus \mathcal{B}(\boldsymbol{a}) \oplus \mathcal{B}(\boldsymbol{b}) \oplus \mathcal{B} \cdot \eta,
$$

where $\mathcal{B}(\boldsymbol{a})$ and $\mathcal{B}(\boldsymbol{b})$ are free $\mathcal{B}$ modules generated by the collections of variables $\boldsymbol{a}=$ $\left(a_{1}, \ldots, a_{g}\right)$ and $\boldsymbol{b}=\left(b_{1}, \ldots, b_{g}\right)$, where $a_{i}$ and $b_{i}$ have bidegrees $(1,1)$ and $(1,0)$ respectively, and $\eta$ has bidegree $(2,1)$. The ring structure is determined by these relations (see (I)):

(I1) $a_{i} \cdot a_{j}=\delta_{i j} \varepsilon a_{i}$

(I2) $b_{i} \cdot b_{j}=0$

(I3) $a_{i} \cdot b_{j}=\delta_{i j} \eta$ 
Here, the element $\varepsilon \in \mathcal{B}^{1,1}$ is a 2 -torsion element in the cohomology of a point that is the image of the symbol $\{-1\} \in K_{1}^{M}(\mathbb{R})$ under the map $K_{1}^{M}(\mathbb{R}) \rightarrow \mathcal{B}^{1,1}$, as shown by the authors in [12].

The relation (1) expresses the geometric fact that the generators $a_{i}$ are equivariant Poincaré duals to the ovals $\alpha_{i}$ (see figure) which cannot be equivariantly moved away from itself, but $2 \alpha_{i}$ can.

The second bigraded ring invariant of a real curve $\Sigma$ that we study is the theory of integral Deligne cohomology for real varieties introduced in [12]. These are invariants of the algebraic structure of the curve that bear the same relation to equivariant ordinary cohomology as the Deligne cohomology for complex varieties has to singular cohomology. In particular, their calculation depends heavily on the previous calculations of equivariant cohomology.

In order to discuss the example of the Deligne cohomology of $\Sigma=\Sigma_{g, g+1}^{1}$, we consider again two sets of variables $\boldsymbol{a}=\left(a_{1}, \ldots, a_{g}\right)$ and $\boldsymbol{b}=\left(b_{1}, \ldots, b_{g}\right)$ having bidegrees $(1,1)$ and $(1,0)$ respectively, and a variable $\eta$ with bidegree $(2,1)$. Let $\mathcal{D}$ be the Deligne cohomology ring of Spec $\mathbb{R}$ and denote by $\widetilde{\mathcal{D}}$ the kernel of the augmentation $\mathcal{D} \rightarrow \mathbb{Z}$. Set $\mathcal{D}(\boldsymbol{b})=\mathcal{D} \otimes \mathbb{Z}(\boldsymbol{b})$, the free $\mathcal{D}$-module generated by the variables $b_{i}$, and define $\tilde{\mathcal{D}}(\boldsymbol{a})=\widetilde{\mathcal{D}} \otimes \mathbb{Z}(\boldsymbol{a})$.

As a module over $\mathcal{D}$, we obtain

$$
H_{\mathcal{D} / \mathbb{R}}^{*}(\Sigma ; \mathbb{Z}(\bullet)) \cong \mathcal{D} \cdot \mathbf{1} \oplus \widetilde{\mathcal{D}}(\boldsymbol{a}) \oplus\left(\mathcal{D}(\boldsymbol{b}) / \mathscr{I}_{\Sigma}\right) \oplus \mathcal{D} \cdot \eta,
$$

where $\mathscr{I}_{\Sigma} \subset \mathcal{D}(\boldsymbol{b})$ is the free abelian group generated by the Jacobian relations, which have the form

$$
\boldsymbol{b} \cdot \hat{\mathrm{X}}=\left\{\sum_{1 \leq i \leq g} \mathrm{X}_{i j} b_{i} \mid 1 \leq j \leq g\right\},
$$

and consist of elements of bidegree $(2,1)$, since $\hat{X}=\left(\mathrm{X}_{i j}\right)$ is a $g \times g$ matrix with entries $\mathrm{X}_{i j} \in \mathcal{D}^{1,1}$ determined by a suitable period matrix for $\Sigma$. The ring structure is determined by these relations:

(I1) $\left(\lambda \otimes a_{i}\right) \cdot\left(\lambda^{\prime} \otimes a_{j}\right)=\delta_{i j}\left(\lambda \lambda^{\prime} \varepsilon\right) \otimes a_{i}$, for all $\lambda, \lambda^{\prime} \in \tilde{\mathcal{D}}$

(I2) $b_{i} \cdot b_{j}=0$

(I3) $\left(\lambda \otimes a_{i}\right) \cdot b_{j}=\delta_{i j} \lambda \eta$

(I4) $x \cdot \eta=0$ for all $x \in \tilde{\mathcal{D}}(\boldsymbol{a}) \oplus\left(\mathcal{D}(\boldsymbol{b}) / \mathscr{I}_{\Sigma}\right)$

Here we use the same notation $\varepsilon \in \mathcal{D}^{1,1}$ for the image of the element $\{-1\} \in K_{1}^{M}(\mathbb{R})$ under the map $K_{1}^{M}(\mathbb{R}) \rightarrow \mathcal{D}^{1,1}$ described in [12]. 
In the general case, the multiplicative structure is complicated by the presence of generators that are not related to ovals, as seen in Theorem 5.12. The resulting ring structure distinguishes curves with isomorphic cohomology modules, such as the curves $\Sigma_{g, r}^{1}$ and $\Sigma_{g, r}^{0}$; see Theorems 4.1 and 5.12.

However, the additive structure of the torsion part of $H_{\mathcal{D} / \mathbb{R}}^{\star}(\Sigma ; \mathbb{Z}(\bullet))$ is easier to describe and we display it in Table 1 below, where we denote by $E=E(\Sigma)$ the number of components of $\Sigma$ without real points. It is interesting to compare this result with the calculations in Pedrini and Weibel [9] where the higher $K$-theory of $\Sigma$ is computed and partial results for the motivic cohomology $H_{\mathcal{M}}^{n}(\Sigma ; \mathbb{Z}(p))$ are obtained. We conjecture that the regulator maps $K_{n}(\Sigma)_{\text {tors }} \rightarrow \prod_{p \geq 0} H_{\mathcal{D} / \mathbb{R}}^{2 p-n}(\Sigma ; \mathbb{Z}(p))_{\text {tors }}$ are injective and the realization maps $H_{\mathcal{M}}^{n}(\Sigma ; \mathbb{Z}(p))_{\text {tors }} \rightarrow H_{\mathcal{D} / \mathbb{R}}^{n}(\Sigma ; \mathbb{Z}(p))_{\text {tors }}$ are isomorphisms for $p>0$. These questions will be addressed in a forthcoming paper.

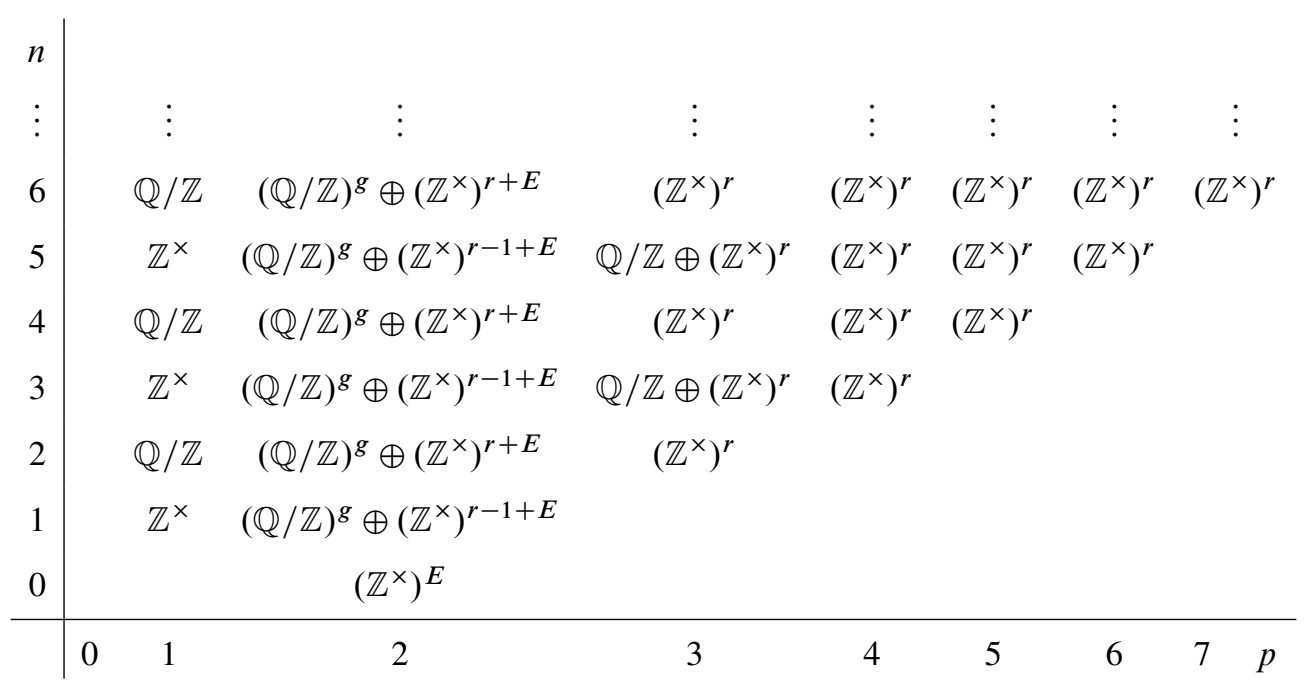

Table 1: Torsion in $H_{\mathcal{D} / \mathbb{R}}^{n}(\Sigma ; \mathbb{Z}(p))$

This paper is organized as follows. In Section 2 we review the necessary topological background, focusing on the equivariant structure of the singular homology groups of real curves. We also give a brief overview of both ordinary equivariant cohomology and integral Deligne cohomology for real varieties [12]. In Section 3 we study the curves of genus 0 and 1. In Section 4 we use the previous cases together with two geometric constructions to obtain the general case of the bigraded equivariant cohomology ring structure for any smooth proper real curve; see Theorem 4.1. In the last section we present in Theorem 5.12 the integral Deligne cohomology ring of these real curves. 


\section{Preliminaries}

\subsection{The topological types of real curves}

Using the terminology in [14], we will often write $\Sigma_{g, r}^{\varepsilon}$ to denote a proper smooth real curve of topological type $\Sigma_{g, r}^{\varepsilon}$. We will also write $\Sigma=\Sigma_{g, r}^{\varepsilon}$ to indicate the type of $\Sigma$.

Definition 2.1 Let $I_{d}$ be the identity matrix of rank $d$, and denote

$$
J_{d}:=\left(\begin{array}{ccc}
0 & \cdots & 1 \\
\vdots & . \cdot & \vdots \\
1 & \cdots & 0
\end{array}\right) .
$$

A curve $\Sigma_{g, r}^{\varepsilon}$, with $g \geq 1$, admits a symplectic basis $\mathfrak{B}:=\left\{\alpha_{1}, \ldots, \alpha_{g} ; \beta_{1}, \ldots, \beta_{g}\right\}$ for $H_{1}^{\operatorname{sing}}(\Sigma(\mathbb{C}) ; \mathbb{Z})$ (that is, a basis such that $\alpha_{i} \cdot \alpha_{j}=\beta_{i} \cdot \beta_{j}=0$ and $\alpha_{i} \cdot \beta_{j}=\delta_{i j}$ ) so that we have $\sigma: H_{1}^{\text {sing }}(\Sigma(\mathbb{C}) ; \mathbb{Z}) \rightarrow H_{1}^{\text {sing }}(\Sigma(\mathbb{C}) ; \mathbb{Z})$ is represented by a matrix of the form

$$
[\sigma]=\left(\begin{array}{cc}
I_{g} & M_{g, r}^{\varepsilon} \\
0 & -I_{g}
\end{array}\right),
$$

where

$$
\begin{aligned}
& M_{g, r}^{1}:=\left(\begin{array}{cc}
J_{g+1-r} & 0 \\
0 & 0
\end{array}\right) \quad \text { and } \quad M_{g, r}^{0}:=\left(\begin{array}{cc}
I_{g+1-r} & 0 \\
0 & 0
\end{array}\right) \text { for } r>0, \\
& M_{g, 0}^{0}:=\left\{\begin{array}{ll}
J_{g} & \text { if } g \text { is even } \\
A & \text { if } g \text { is odd }
\end{array} \text { for } r=0, \text { where } A:=\left(\begin{array}{cc}
J_{g-1} & 0 \\
0 & 0
\end{array}\right) .\right.
\end{aligned}
$$

We call a basis satisfying these conditions a real basis for the first homology group.

2.1.1 Separating case If $\Sigma=\Sigma_{g, r}^{1}$ is a separating curve, then $\Sigma(\mathbb{C})$ can be visualized as in Figure 2, where the action of $\mathfrak{S}$ is given by reflection with respect to the horizontal plane.

Denoting by $A_{i}, B_{j}, C_{k}, D_{k} \in H_{1}^{\text {sing }}(\Sigma(\mathbb{C}), \mathbb{Z}), i=1, \ldots, r, j=1, \ldots, r-1, k=$ $1, \ldots, q, 2 q=g+1-r$, the classes in singular homology of the circles indicated in the figure, one can write

$$
H_{1}^{\text {sing }}(\Sigma(\mathbb{C}), \mathbb{Z})=\mathbb{Z}\{\boldsymbol{A}, \boldsymbol{B}, \boldsymbol{C}, \sigma \boldsymbol{C}, \boldsymbol{D}, \sigma \boldsymbol{D}\},
$$

where $\boldsymbol{A}$ denotes the ordered set of homology classes $\left(A_{1}, \ldots, A_{r-1}\right)$. The sets $\boldsymbol{B}, \boldsymbol{C}$ and $\boldsymbol{D}$ are defined similarly. Also $\sigma \boldsymbol{C}:=\left(\sigma C_{1}, \ldots, \sigma C_{q}\right)$ and similarly for $\sigma \boldsymbol{D}$. Note that $\sigma \boldsymbol{A}=\boldsymbol{A}$ and $\sigma \boldsymbol{B}=-\boldsymbol{B}$. 


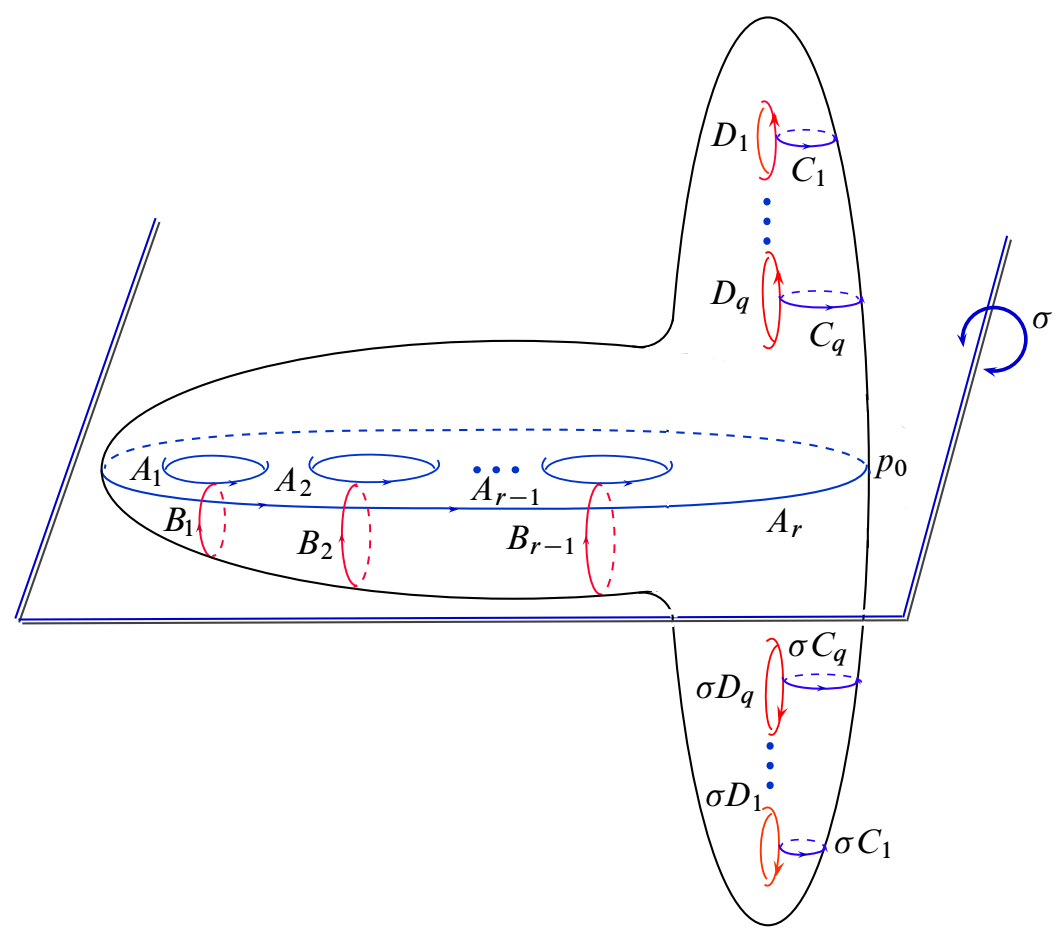

Figure 2: Curve of separating type $\Sigma_{g, r}^{1}$

It is easily seen that the elements $\alpha_{1}, \ldots, \alpha_{g} ; \beta_{1}, \ldots, \beta_{g}$ introduced in Table 2 form a real basis for $H_{1}^{\text {sing }}(\Sigma(\mathbb{C}), \mathbb{Z})$ according to Definition 2.1 .

$$
\begin{aligned}
& \alpha_{1}=C_{1}+\sigma C_{1} \quad \beta_{1}=D_{1} \\
& \alpha_{q}=C_{q}+\sigma C_{q} \quad \beta_{q}=D_{q} \\
& \alpha_{q+1}=D_{q}+\sigma D_{q} \quad \beta_{q+1}=\sigma C_{q} \\
& \alpha_{2 q}=D_{1}+\sigma D_{1} \quad \beta_{2 q}=\sigma C_{1} \\
& \alpha_{2 q+1}=A_{1} \quad \beta_{2 q+1}=B_{1} \\
& \alpha_{2 q+r-1}=A_{r-1} \quad \beta_{2 q+r-1}=B_{r-1}
\end{aligned}
$$

Table 2: Real basis for $H_{1}^{\text {sing }}\left(\Sigma_{g, r}^{1}(\mathbb{C}), \mathbb{Z}\right)$ 
2.1.2 Nonseparating case without real points Let us first consider the case $\Sigma_{g, 0}^{0}$, where the curve has no real points, which can be visualized in Figure 3. In this case, the action of $\mathfrak{S}$ can be seen as reflection across the origin $\boldsymbol{o}$, and a real basis $\left\{\alpha_{1}, \ldots, \alpha_{g} ; \beta_{1}, \ldots, \beta_{g}\right\}$ as in Definition 2.1 is displayed in Table 3.
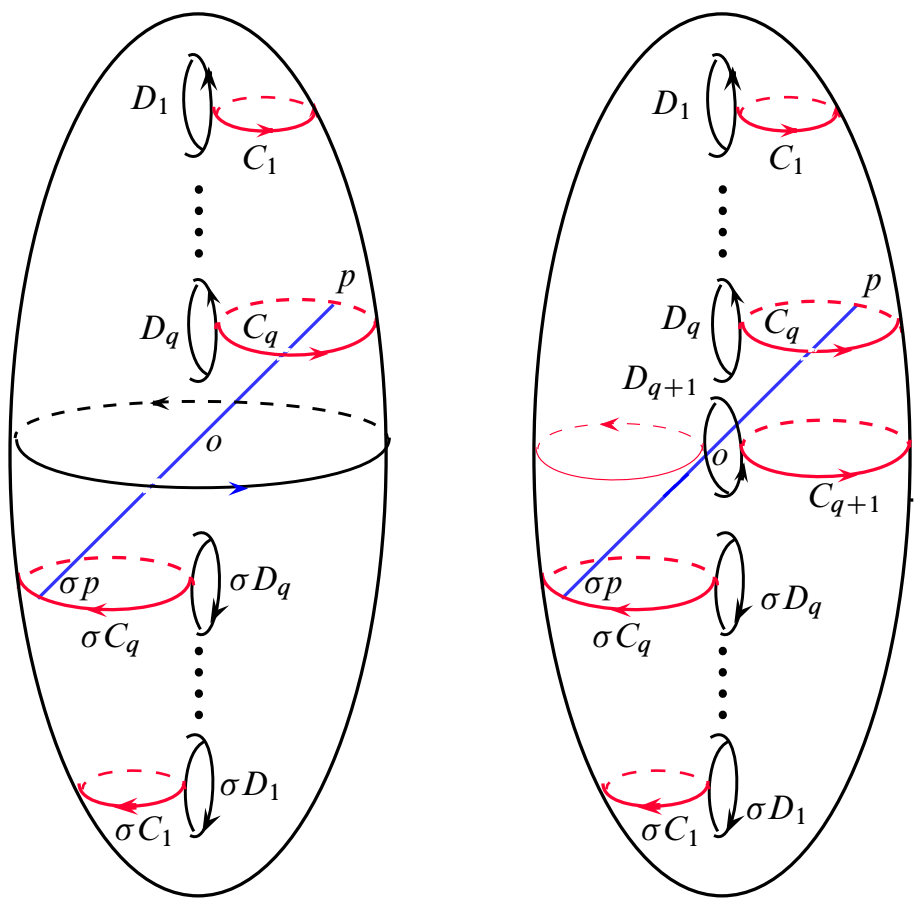

Figure 3: Curves with no real points $\Sigma_{g, 0}^{0}$

\begin{tabular}{c|c}
\multicolumn{2}{c}{$g=2 q$} \\
\hline$\alpha_{1}=C_{1}+\sigma C_{1}$ & $\beta_{1}=D_{1}$ \\
$\vdots$ & $\vdots$ \\
$\alpha_{q}=C_{q}+\sigma C_{q}$ & $\beta_{q}=D_{q}$ \\
\hline$\alpha_{q+1}=D_{q}+\sigma D_{q}$ & $\beta_{q+1}=\sigma C_{q}$ \\
$\vdots$ & $\vdots$ \\
$\alpha_{2 q}=D_{1}+\sigma D_{1}$ & $\beta_{2 q}=\sigma C_{1}$
\end{tabular}

\begin{tabular}{c|c}
$g=2 q+1$ \\
\hline$\alpha_{1}=C_{1}+\sigma C_{1}$ & $\beta_{1}=D_{1}$ \\
$\vdots$ & $\vdots$ \\
$\alpha_{q}=C_{q}+\sigma C_{q}$ & $\beta_{q}=D_{q}$ \\
\hline$\alpha_{q+1}=D_{q}+\sigma D_{q}$ & $\beta_{q+1}=\sigma C_{q}$ \\
$\vdots$ & $\vdots$ \\
$\alpha_{2 q}=D_{1}+\sigma D_{1}$ & $\beta_{2 q}=\sigma C_{1}$ \\
\hline$\alpha_{2 q+1}=-D_{q+1}$ & $\beta_{2 q+1}=C_{q+1}$
\end{tabular}

Table 3: Real basis for $H_{1}^{\text {sing }}\left(\Sigma_{g, 0}^{0}(\mathbb{C}), \mathbb{Z}\right)$ 
2.1.3 Nonseparating case with real points The case of nonseparating curves with real points $\Sigma=\Sigma_{g, r}^{0}$, with $r>0$ is harder to visualize, and we postpone its description to Section 4.2, limiting the presentation here to the case $g=1$. Choosing a real point $\mathbf{0}$ in $\Sigma$ allows one to present $\Sigma_{1,1}^{0}(\mathbb{C})$ as an elliptic curve $\mathbb{C} / \Lambda$, whose fundamental domain is shown in Figure 4. It is clear that $\left\{\alpha_{1}=\alpha, \beta_{1}=\beta\right\}$ is a real basis for $H_{1}^{\text {sing }}(\Sigma(\mathbb{C}), \mathbb{Z})$, as in Definition 2.1.
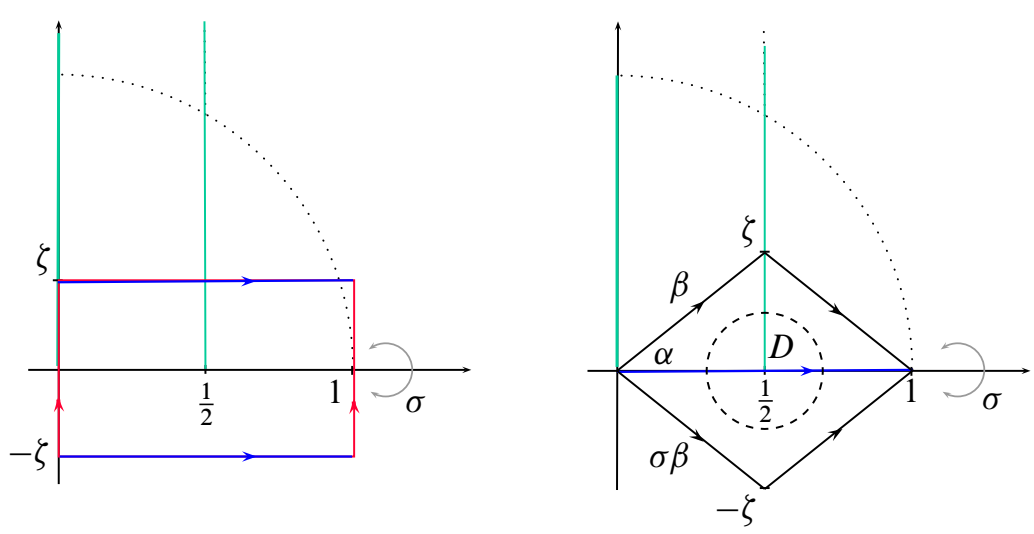

Figure 4: Real elliptic curves $\Sigma_{1,2}^{1}$ and $\Sigma_{1,1}^{0}$

\subsection{Holomorphic differentials}

Given a subring $A \subset \mathbb{R}, p \in \mathbb{Z}$ and a $\mathfrak{S}$-space $X$, let $H_{\text {sing }}^{j}(X ; A(p))$ be the singular cohomology groups of $X$ with coefficients in the $\mathbb{Z}[\mathfrak{S}]$-submodule $A(p):=$ $(2 \pi \sqrt{-1})^{p} A \subset \mathbb{C}$. The simultaneous action of $\mathfrak{S}$ on $X$ and $A(p)$ then induces an involution

$$
\sigma_{\infty}: H_{\text {sing }}^{j}(X ; A(p)) \rightarrow H_{\text {sing }}^{j}(X ; A(p)) .
$$

The cup product and the evident pairings $A(p) \otimes A(q) \rightarrow A(p+q)$ give

$$
H_{\text {sing }}^{*}(X ; A(\bullet)):=\bigoplus_{r \geq 0, p \in \mathbb{Z}} H_{\text {sing }}^{r}(X, A(p)) \text {, }
$$

the structure of a bigraded ring, where the elements in $H_{\text {sing }}^{r}(X, A(p))$ have degree $(r, p)$. The invariants $H_{\text {sing }}^{*}(X ; A(\bullet))^{\mathfrak{S}}$ form a bigraded subring of $H_{\text {sing }}^{*}(X ; A(\bullet))$ and, for simplicity, we denote

$$
\mathfrak{h}_{\mathbb{R}}^{j}(X):=\left(H_{\text {sing }}^{j}(X ; \mathbb{C})\right)^{\mathfrak{S}} .
$$




\section{Notation 2.2 Let}

$$
\vartheta: H_{j}^{\text {sing }}(\Sigma(\mathbb{C}) ; \mathbb{Z}) \stackrel{\cong}{\rightarrow} H_{\text {sing }}^{2-j}(\Sigma(\mathbb{C}) ; \mathbb{Z})
$$

denote the Poincaré duality isomorphism, satisfying $\alpha=\vartheta_{\alpha} \cap[\Sigma(\mathbb{C})]$, where $[\Sigma(\mathbb{C})]$ is the fundamental class determined by the choice of $\sqrt{-1}$, and let $\eta_{o}:=\vartheta_{[\mathrm{pt}]} \in$ $H_{\text {sing }}^{2}(\Sigma(\mathbb{C}) ; \mathbb{Z})$ be the orientation class.

Given $\Sigma=\Sigma_{g, r}^{\varepsilon}$, consider a real basis $\mathfrak{B}$ for $H_{1}^{\text {sing }}(\Sigma(\mathbb{C}), \mathbb{Z})$ as in Definition 2.1 and denote $M=M_{g, r}^{\varepsilon}$. Let $\left\{\omega_{1}, \ldots, \omega_{g}\right\}$ be a basis of holomorphic differentials adapted to $\mathfrak{B}$, whose period matrix with respect to $\mathcal{B}$ has the form $\left(I_{g} \mid Z\right)$.

\section{Definition 2.3 Define}

$$
\mathrm{e}_{j}:=\omega_{j}-\bar{\omega}_{j} \quad \text { and } \quad \mathrm{f}_{j}:=-\left(\omega_{j}+\bar{\omega}_{j}\right), \quad j=1, \ldots, g,
$$

and write $\boldsymbol{\omega}=\left(\omega_{1}, \ldots, \omega_{g}\right), \mathbf{e}=\left(\mathrm{e}_{1}, \ldots, \mathrm{e}_{g}\right)$ and $\mathbf{f}=\left(\mathrm{f}_{1}, \ldots, \mathrm{f}_{g}\right)$, seen as $1 \times g$ matrices of cohomology classes.

The involution $\sigma$ reverses orientation, ie $\sigma_{*}[\Sigma(\mathbb{C})]=-[\Sigma(\mathbb{C})]$, implying that

$$
\sigma^{*} \vartheta_{\alpha}=-\vartheta_{\sigma_{*} \alpha}
$$

This simple fact has many relevant consequences, such as the next result (whose proof is left to the reader). Here we consider $H_{\text {sing }}^{j}(\Sigma(\mathbb{C}) ; \mathbb{Z})$ as a subgroup of $H_{\text {sing }}^{j}(\Sigma(\mathbb{C}) ; \mathbb{C})$ since the former is free, and identify the latter with deRham cohomology. Under this identification one has $\sigma_{\infty}=\sigma$ on $H_{\text {sing }}^{j}(\Sigma(\mathbb{C}) ; \mathbb{Z})$.

Proposition 2.4 Let $\Sigma$ and $\mathcal{B}$ be as above and denote

$$
\vartheta_{\boldsymbol{\alpha}}=\left(\vartheta_{\alpha_{1}}, \ldots, \vartheta_{\alpha_{g}}\right) \quad \text { and } \quad \vartheta_{\boldsymbol{\beta}}=\left(\vartheta_{\beta_{1}}, \ldots, \vartheta_{\beta_{g}}\right)
$$

Then:

(i) $\boldsymbol{\omega}=\vartheta_{\boldsymbol{\alpha}} \cdot Z-\vartheta_{\boldsymbol{\beta}}$, where $Z$ is the period matrix with respect to $\mathcal{B}$.

(ii) The period matrix has the form $Z=\frac{1}{2} M+i \mathrm{~T}$, where $\mathrm{T}$ is a symmetric positive definite real matrix.

(iii) The adapted holomorphic differentials are "real", ie invariant under the involution $\sigma_{\infty}$; equivalently, $\sigma^{*} \omega=\overline{\boldsymbol{\omega}}$.

(iv) $\mathbf{f}=\boldsymbol{\vartheta}_{\boldsymbol{\beta}}+\sigma_{\infty} \boldsymbol{\vartheta}_{\boldsymbol{\beta}}$ and $\mathbf{e}=2 \boldsymbol{i} \boldsymbol{\vartheta}_{\boldsymbol{\alpha}} \mathrm{T}$. 
It follows that the collections $\left\{\omega_{1}, \ldots, \omega_{g}, \mathrm{e}_{1}, \ldots, \mathrm{e}_{g}\right\}$ and $\left\{\omega_{1}, \ldots, \omega_{g} ; \mathrm{f}_{1}, \ldots, \mathrm{f}_{j}\right\}$ are bases for $\mathfrak{h}_{\mathbb{R}}^{1}(\Sigma(\mathbb{C}))$ that are related by

$$
(\boldsymbol{\omega}, \mathbf{e})=(\boldsymbol{\omega}, \mathbf{f})\left(\begin{array}{cc}
I_{g} & 2 I_{g} \\
0 & I_{g}
\end{array}\right)
$$

\subsection{Ordinary $\mathrm{RO}(\mathfrak{S})$-graded equivariant cohomology}

In [1] Bredon defines an equivariant cohomology theory $H_{G}^{n}(X ; M)$ for $G$-spaces, where $G$ is a finite group and $\underline{M}$ is a contravariant coefficient system. When $\underline{M}$ is a Mackey functor, P May et al [6] showed that this theory can be uniquely extended to an $\mathrm{RO}(G)$-graded theory $\left\{H_{G}^{\alpha}(X ; \underline{M}), \alpha \in \mathrm{RO}(G)\right\}$, called $\mathrm{RO}(G)$-graded ordinary equivariant cohomology theory, where $\operatorname{RO}(G)$ denotes the orthogonal representation ring of $G$. When $G=\mathfrak{S}$, one has $\operatorname{RO}(\mathfrak{S})=\mathbb{Z} \cdot \mathbf{1} \oplus \mathbb{Z} \cdot \xi$, where $\mathbf{1}$ is the trivial representation and $\xi$ is the sign representation. In this paper we use the motivic notation

$$
H_{\mathrm{Br}}^{n, p}(X, \underline{M}):=H_{\mathfrak{S}}^{(n-p) \cdot \mathbf{1}+p \cdot \xi}(X ; \underline{M}),
$$

and call $H_{\mathrm{Br}}^{n, p}(X, \underline{M})$ bigraded Bredon cohomology.

Remark 2.5 Bredon cohomology comes with a natural cup product $U$ that makes $H_{\mathrm{Br}}^{*, \bullet}(X ; \underline{\mathbb{Z}})$ into a graded-commutative bigraded ring, in the sense that

$$
a \cup b=(-1)^{r+r^{\prime}} b \cup a, \quad \text { if } \quad \operatorname{deg} a=(r, p) \quad \text { and } \quad \operatorname{deg} b=\left(r^{\prime}, p^{\prime}\right) .
$$

We simply denote $a b:=a \cup b$, keeping in mind the graded commutativity as above.

Notation 2.6 (Equivariant spheres) Given $n \geq p$ we will denote by $S^{n, p}$ the sphere of the representation $V^{n, p}:=\mathbf{1}^{n-p}+\xi^{p}$ (that is, $S^{n, p}=V^{n, p} \cup\{\infty\}$ ), and by $S_{a}^{n}$ the unit sphere of the representation $\xi^{n}$, which is the sphere $S^{n}$ under the antipodal map.

As with other cohomology theories, there is a reduced version of bigraded Bredon cohomology, which for a based $\mathfrak{S}$-space $\left(X, x_{0}\right)$ can be given by

$$
\tilde{H}_{\mathrm{Br}}^{*, \bullet}(X ; \underline{\mathbb{Z}}):=\operatorname{ker}\left(H_{\mathrm{Br}}^{*, \bullet}(X ; \underline{\mathbb{Z}}) \rightarrow H_{\mathrm{Br}}^{*, \bullet}\left(x_{0} ; \underline{\mathbb{Z}}\right)\right) .
$$

The map in cohomology induced by the inclusion $x_{0} \rightarrow X$ gives a splitting

$$
H_{\mathrm{Br}}^{*, \bullet}(X ; \underline{\mathbb{Z}})=\tilde{H}_{\mathrm{Br}}^{*, \bullet}(X ; \underline{\mathbb{Z}}) \oplus H_{\mathrm{Br}}^{*, \bullet}\left(x_{0} ; \underline{\mathbb{Z}}\right) .
$$


For any equivariant cohomology theory $\mathfrak{H}^{*, \bullet}$ one can define its Borel version $\mathfrak{H}_{\text {bor }}^{*, \bullet}$ as $\mathfrak{H}_{\text {bor }}^{*, \bullet}(U):=\mathfrak{H}^{*, \bullet}(U \times E \mathfrak{S})$. In particular, one has an associated Borel cohomology theory $H_{\text {bor }}^{n, p}(X, \underline{\mathbb{Z}}):=H_{\mathrm{Br}}^{n, p}(X \times E \mathfrak{S} ; \underline{\mathbb{Z}})$, which is $(0,2)$ periodic; see the authors [10].

\subsubsection{The forgetful functor There are natural forgetful homomorphisms}

$$
\varphi: H_{\mathrm{Br}}^{r, p}(X ; \underline{\mathbb{Z}}) \longrightarrow H_{\text {sing }}^{r}(X ; \mathbb{Z}(p))^{\mathfrak{S}},
$$

giving a homomorphism $\varphi: H_{\mathrm{Br}}^{*, \bullet}(X ; \underline{\mathbb{Z}}) \longrightarrow H_{\text {sing }}^{*}(X ; \mathbb{Z}(\bullet))^{\mathfrak{S}}$ of bigraded rings.

Remark 2.7 Choosing $i=\sqrt{-1}$ one can identify $\mathbb{Z}(\bullet)$ with the ring of Laurent polynomials $\mathbb{Z}\left[\xi, \xi^{-1}\right]$, where $\operatorname{deg} \xi=(0,1)$, as a $\mathbb{Z}[\mathfrak{S}]$-algebra under $\sigma: \xi \mapsto-\xi$. In particular, $H_{\text {sing }}^{*}(\mathrm{pt} ; \mathbb{Z}(\bullet)) \equiv \mathbb{Z}\left[\xi, \xi^{-1}\right]$ as a $\mathfrak{S}$-algebra whose invariants are $\mathbb{Z}\left[\xi^{2}, \xi^{-2}\right]$.

Given a $\mathfrak{S}$-space $X$, the natural projection $X \times \mathfrak{S} \stackrel{\pi}{\rightarrow} X$ induces a commutative diagram of bigraded cohomology rings:

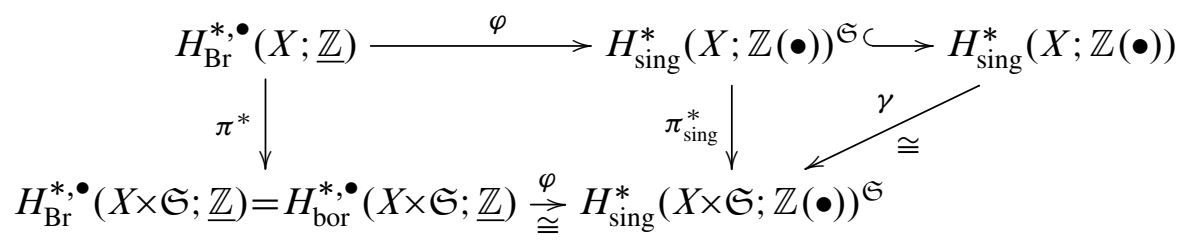

Writing $H_{\text {sing }}^{*}(X \times \mathfrak{S} ; \mathbb{Z}(\bullet)) \equiv H_{\text {sing }}^{*}(X ; \mathbb{Z}(\bullet)) \times H_{\text {sing }}^{*}(X ; \mathbb{Z}(\bullet))$, the isomorphism $\gamma$ is given by sending $\boldsymbol{a} \in H_{\text {sing }}^{r}(X ; \mathbb{Z}(p))$ to $\gamma(\boldsymbol{a}):=\left(\boldsymbol{a},(-1)^{p} \sigma^{*} \boldsymbol{a}\right)$. The equality in the left lower corner follows from the fact the canonical projection $X \times \mathfrak{S} \times E \mathfrak{S} \rightarrow X \times \mathfrak{S}$ is an equivariant homotopy equivalence.

\subsection{Poincaré duality}

There is a bigraded Bredon homology theory, denoted $H_{*, \bullet}^{\mathrm{Br}}(-; \underline{\mathbb{Z}})$, for which Poincaré duality holds.

Proposition 2.8 [10, Proposition 1.13] Let $X$ be a connected, proper real smooth variety of dimension $n$. Then, there is a class $[X] \in H_{2 n, n}^{\mathrm{Br}}(X ; \underline{\mathbb{Z}})$, called the fundamental homology class, such that the cap product with $[X]$ gives the $\mathfrak{S}$-equivariant version of the Poincaré duality isomorphism:

$$
\text { PD: } H_{\mathrm{Br}}^{k, l}(X ; \underline{\mathbb{Z}}) \rightarrow H_{2 n-k, n-l}^{\mathrm{Br}}(X ; \underline{\mathbb{Z}})
$$

for each $k, l \in \mathbb{Z}$. 


\subsection{Integral Deligne cohomology for real varieties}

In [12] an integral version of Deligne cohomology for real varieties was developed. To a real smooth proper real variety $X$ it assigns a bigraded ring $H_{\mathcal{D} / \mathbb{R}}^{*}(X ; \mathbb{Z}(\bullet))$ satisfying the following properties

(1) One has a long exact sequence

$$
\begin{aligned}
\cdots & \rightarrow H_{\text {sing }}^{j-1}(X(\mathbb{C}), \mathbb{C})^{\mathfrak{S}} \stackrel{\delta_{\mathbb{R}}}{\rightarrow} H_{\mathcal{D} / \mathbb{R}}^{j}(X ; \mathbb{Z}(p)) \\
& \stackrel{\nu}{\rightarrow} H_{\mathrm{Br}}^{j, p}(X(\mathbb{C}) ; \underline{\mathbb{Z}}) \oplus\left\{F^{p} H_{\text {sing }}^{j}(X(\mathbb{C}) ; \mathbb{C})\right\}^{\mathfrak{S}} \rightarrow H_{\text {sing }}^{j}(X(\mathbb{C}) ; \mathbb{C})^{\mathfrak{S}} \rightarrow \cdots,
\end{aligned}
$$

where $X(\mathbb{C})$ denotes the set of complex points of $X$ with the analytic topology and $\left\{F^{p} H^{j}(X(\mathbb{C}) ; \mathbb{C})\right\}^{\mathfrak{S}}$ denotes the invariants of the $p^{\text {th }}$ level of the Hodge filtration on singular cohomology under the action of $\sigma_{\infty}$.

(2) If $X_{\mathbb{C}}$ is a smooth proper complex variety and $X_{\mathbb{C} / \mathbb{R}}$ denotes $X_{\mathbb{C}}$ considered as a real variety, there are natural isomorphisms $H_{\mathcal{D} / \mathbb{R}}^{i}\left(X_{\mathbb{C} / \mathbb{R}} ; \mathbb{Z}(p)\right) \cong$ $H_{\mathcal{D} / \mathbb{C}}^{i}\left(X_{\mathbb{C}} ; \mathbb{Z}(p)\right)$, where the latter denotes the usual Deligne cohomology of $X_{\mathbb{C}}$.

(3) If $X_{\mathbb{C}}$ is the complex variety obtained from $X$ by base extension, the corresponding map of real varieties $X_{\mathbb{C} / \mathbb{R}} \rightarrow X$ induces natural homomorphisms $H_{\mathcal{D} / \mathbb{R}}^{j}(X ; \mathbb{Z}(p)) \rightarrow H_{\mathcal{D} / \mathbb{C}}^{j}\left(X_{\mathbb{C}} ; \mathbb{Z}(p)\right)^{\mathfrak{S}}$, where the latter denotes the invariants of the Deligne cohomology of $X_{\mathbb{C}}$.

(4) The map $\Psi_{\mathcal{D}}: H_{\mathcal{D} / \mathbb{R}}^{*}(X ; \mathbb{Z}(\bullet)) \rightarrow H_{\mathcal{D} / \mathbb{C}}^{*}\left(X_{\mathbb{C}} ; \mathbb{Z}(\bullet)\right)^{\mathfrak{S}}$ above and the map $\Psi_{\mathcal{B}}: H_{\mathcal{D} / \mathbb{R}}^{*}(X ; \mathbb{Z}(\bullet)) \rightarrow H_{\mathrm{Br}}^{*, \bullet}(X ; \underline{\mathbb{Z}})$ of $(12)$ are ring homomorphisms.

(5) With negative weights $p<0, H_{\mathcal{D} / \mathbb{R}}^{*}(X ; \mathbb{Z}(p))$ is defined to coincide with ordinary equivariant cohomology.

(6) For $p=1$, one has $H_{\mathcal{D} / \mathbb{R}}^{j}(X ; \mathbb{Z}(1)) \cong H^{j-1}\left(X ; \mathcal{O}^{\times}\right)$.

Just as Bredon cohomology has its Borel counterpart, so does integral Deligne cohomology for real varieties. This is explained in [12, Appendix A]. This counterpart coincides with the cohomology theory proposed by Esnault and Viehweg in [4] and for this reason we call it Esnault-Viehweg-Deligne cohomology and denote it $H_{\mathcal{D} / \mathbb{R}, E V}^{*}(X ; \mathbb{Z}(\bullet))$.

\subsection{The cohomology rings of a point and related algebras}

In what follows, we consider $\mathbb{R} / \mathbb{Z}, \mathbb{R}$ and $\mathbb{R}^{\times}$as bigraded groups concentrated in degree $(0,0)$. We start with a list of variables that appear in the presentation of various cohomology rings of a point. The context will determine which rings are under consideration. 


\subsubsection{The Bredon cohomology ring of a point Consider the ring}

$$
\mathcal{B}_{0}=\mathbb{Z}[\varepsilon, \tau] \cong \mathbb{Z}[e, \tau] /\langle 2 e\rangle
$$

and note that $\mathbb{Z}[\varepsilon, \tau]$ is not a polynomial ring over $\mathbb{Z}$, since $\varepsilon$ is a torsion element. We can also write $\mathcal{B}_{0}$ as $\mathcal{B}_{0}=\mathbb{Z}[\tau] \oplus \varepsilon \mathbb{F}_{2}[\varepsilon, \tau]$, where the second summand is the ideal generated by $\varepsilon$ in $\mathcal{B}_{0}$. Now, let $\mathbb{Z}\left[\tau^{-1}\right] \cdot \alpha$ and $\mathbb{F}_{2}\left[\varepsilon^{-1}, \tau^{-1}\right] \cdot \theta$ be free modules over the indicated polynomial rings, whose generators have their degrees displayed in Table 4, and denote

$$
\mathcal{B}_{-}=\mathbb{Z}\left[\tau^{-1}\right] \cdot \alpha \oplus \mathbb{F}_{2}\left[\varepsilon^{-1}, \tau^{-1}\right] \cdot \theta .
$$

The Bredon cohomology of a point $\mathcal{B}:=H_{\mathrm{Br}}^{*, \bullet}(\mathrm{pt} ; \underline{\mathbb{Z}})$ can be written as a direct sum of abelian groups

$$
\mathcal{B}=\mathcal{B}_{0} \oplus \mathcal{B}_{-}=\mathbb{Z}[\tau] \oplus \varepsilon \mathbb{F}_{2}[\varepsilon, \tau] \oplus \mathbb{Z}\left[\tau^{-1}\right] \cdot \alpha \oplus \mathbb{F}_{2}\left[\varepsilon^{-1}, \tau^{1}\right] \cdot \theta .
$$

The product structure on $\mathcal{B}$ is determined by the fact that $\mathcal{B}_{0}$ is a subring and by the relations

$$
\alpha \cdot \tau=2, \quad \alpha \cdot \theta=\alpha \cdot \varepsilon=\theta \cdot \tau=\theta \cdot \varepsilon=0 .
$$

Note that $\mathcal{B}$ is not finitely generated as a ring, and that $\mathcal{B}$ has no homogeneous elements in degrees $(p, q)$ when $p \cdot q<0$.

\begin{tabular}{c|cccccccc} 
variable & $\varepsilon$ & $\varepsilon^{-1}$ & $\tau$ & $\tau^{-1}$ & $\alpha$ & $\theta$ & $\mathrm{X}$ & $\mathrm{Y}$ \\
degree & $(1,1)$ & $(-1,-1)$ & $(0,2)$ & $(0,-2)$ & $(0,-2)$ & $(0,-3)$ & $(1,1)$ & $(1,0)$ \\
type & torsion & torsion & & & & torsion & & \\
& $2 \varepsilon=0$ & $2 \varepsilon^{-1}=0$ & & & & $2 \theta=0$ & &
\end{tabular}

Table 4: Variables and their degrees for the cohomology of a point

Remark 2.9 (1) Under the isomorphism $H_{\text {sing }}^{*}(\mathrm{pt} ; \mathbb{Z}(\bullet)) \cong \mathbb{Z}\left[\xi, \xi^{-1}\right]$ explained in Remark 2.7, the forgetful functor $\varphi: \mathcal{B} \rightarrow \mathbb{Z}\left[\xi, \xi^{-1}\right]$ is determined by $\varphi(\tau)=\xi^{2}$ and $\varphi(\alpha)=2 \xi^{-2}$. Its image is the subring

$$
\mathbb{Z}\left[\xi^{2}\right]+\left(2 \xi^{-2}\right) \mathbb{Z}\left[\xi^{-2}\right] \subset \mathbb{Z}\left[\xi^{2}, \xi^{-2}\right] \subset \mathbb{Z}\left[\xi, \xi^{-1}\right] .
$$

(2) The torsion ideal in $\mathcal{B}$ is precisely $\mathcal{B}_{\text {tor }}=\varepsilon \mathbb{F}_{2}[\varepsilon, \tau] \oplus \mathbb{F}_{2}\left[\varepsilon^{-1}, \tau^{-1}\right] \cdot \theta$.

2.6.2 The associated Borel cohomology ring of a point The associated Borel cohomology ring $\mathcal{A}:=H_{\text {bor }}^{*, \bullet}(\mathrm{pt}, \underline{\mathbb{Z}})$ of a point has a simpler description than $\mathcal{B}$, due to its $(0,2)$ periodicity, and it can also be seen as the localization of $\mathcal{B}$ at $\tau$. More precisely,

$$
\mathcal{A} \cong \mathbb{Z}\left[\varepsilon, \tau, \tau^{-1}\right]
$$


The map $\mathcal{B} \rightarrow \mathcal{A}$ induces the natural inclusion of $\mathcal{B}_{0}=\mathbb{Z}[\tau, \varepsilon]$ into $\mathcal{A}$, sends the element $\alpha$ to $2 \tau^{-1}$ and sends $\theta$ to 0 .

The forgetful functor $\varphi: \mathcal{A} \rightarrow \mathbb{Z}\left[\xi, \xi^{-1}\right]$ is determined by $\varphi(\tau)=\xi^{2}$.

2.6.3 The integral Deligne cohomology $\operatorname{ring}$ of $\operatorname{Spec} \mathbb{R}$ Let $\langle\varepsilon\rangle \subset \mathcal{B}_{0}$ denote the ideal generated by $\varepsilon \in \mathcal{B}^{1,1}$. It is easy to see that $\langle\varepsilon\rangle \cong \varepsilon \mathbb{F}_{2}[\varepsilon, \tau]$ and hence we can consider $\mathbb{Z} \oplus \varepsilon \mathbb{F}_{2}[\varepsilon, \tau]$ as a subring of $\mathcal{B}_{0}$.

Define

$$
M=\mathbb{R}[\tau] \cdot \mathrm{E} \oplus(\tau \mathbb{Z}[\tau] \cdot \mathrm{Y}) \otimes \mathbb{R} / \mathbb{Z},
$$

where $\mathrm{E}$ and $\mathrm{Y}$ are generators of degree $(1,1)$ and $(1,0)$, respectively, and give

$$
\mathcal{D}_{0}:=\mathbb{Z} \oplus \underbrace{\varepsilon \mathbb{F}_{2}[\varepsilon, \tau] \oplus M}_{\mathcal{D}_{+}}
$$

a ring structure so that $\mathbb{Z} \oplus \varepsilon \mathbb{F}_{2}[\varepsilon, \tau]$ is a subring, and $\mathcal{D}_{+} \cdot M=\{0\}$.

The full cohomology ring $\mathcal{D}=H_{\mathcal{D} / \mathbb{R}}^{*}(\operatorname{Spec} \mathbb{R} ; \mathbb{Z}(\bullet))$ has the form

$$
\mathcal{D}=\mathcal{D}_{0} \oplus \mathcal{B}_{-},
$$

where $\mathcal{B}_{-}$was introduced in (14), $\mathcal{D}_{0}$ is a subring and $\mathcal{D}_{+} \cdot \mathcal{B}_{-}=\{0\}$. We denote by $\widetilde{\mathcal{D}} \subset \mathcal{D}$ the ideal $\mathcal{D}_{+} \oplus \mathcal{B}_{-}$so that $\mathcal{D}=\mathbb{Z} \oplus \widetilde{\mathcal{D}}$.

Remark 2.10 The following observations are important for subsequent calculations and to relate this presentation of $\mathcal{D}$ with the one in [12].

(a) The ideal $M$ of $\mathcal{D}$ satisfies $M^{2}=0$.

(b) Define $J=\varepsilon \mathbb{F}_{2}[\varepsilon, \tau] \oplus \mathbb{F}_{2}\left[\varepsilon^{-1}, \tau^{-1}\right] \cdot \theta \subset \mathcal{D}$. Then $J$ is an ideal in $\mathcal{D}$ and the forgetful functor $\varphi: \mathcal{D} \rightarrow \mathcal{B}$ sends $J$ isomorphically onto $\mathcal{B}_{\text {tor }}$. It is easy to see that, for each $k \geq 1$ one has $J^{k}=\varepsilon^{k} \mathbb{F}_{2}[\varepsilon, \tau] \oplus \mathbb{F}_{2}\left[\varepsilon^{-1}, \tau^{-1}\right] \cdot \theta$.

(c) As explained in Remark 2.7, the choice of $i=\sqrt{-1}$ allows for the identifications $\xi \equiv 2 \pi \boldsymbol{i}$ and $\xi^{2} \equiv \tau \equiv(2 \pi \boldsymbol{i})^{2}$ and, in particular, $\mathbb{Z}(p) \equiv \mathbb{Z} \cdot \xi^{p}$. We can go further and identify $\mathcal{D}^{1,2 r+1} \equiv \mathbb{R}^{\times}$under the isomorphism

$$
\begin{aligned}
\boldsymbol{e}_{r}: \mathcal{D}^{1,2 r+1}=\mathbb{F}_{2} \cdot\left\{\varepsilon \tau^{r}\right\} \oplus \mathbb{R} \cdot\left\{\tau^{r} \mathrm{E}\right\} & \longrightarrow \mathbb{R}^{\times}, \\
\left(\delta \cdot \varepsilon \tau^{r} \cdot, \lambda \tau^{r} \mathrm{E}\right) & \longmapsto \exp (\lambda+\delta \pi \boldsymbol{i}),
\end{aligned}
$$

where $\delta \in\{0,1\}$. In a similar fashion, we identify $\mathcal{D}^{1,2 r} \equiv \mathbb{R} / \mathbb{Z}(2 r)$ by sending $[t] \otimes \tau^{r} \cdot \mathrm{Y} \rightarrow\left[t(2 \pi \boldsymbol{i})^{2 r}\right]$. In both cases, one obtains an identification $\mathcal{D}^{1, j} \equiv$ $(\mathbb{C} / \mathbb{Z}(j))^{\mathfrak{S}}=\left(\mathcal{D}_{\mathbb{C}}\right)^{\mathfrak{S}}$. 
2.6.4 The ring $\mathcal{D}_{\mathrm{EV}}$ The Esnault-Viehweg-Deligne cohomology of Spec $\mathbb{R}$

$$
\mathcal{D}_{\mathrm{EV}}:=H_{\mathcal{D} / \mathbb{R}}^{*}(E \mathfrak{S} ; \mathbb{Z}(\bullet)),
$$

explained in Section 2.5, has the following description. Start with $\mathbb{Z}\left[\tau^{-1}\right] \oplus \varepsilon \mathbb{F}_{2}\left[\varepsilon, \tau, \tau^{-1}\right]$ seen as a subring of $\mathcal{A}=\mathbb{Z}\left[\varepsilon, \tau, \tau^{-1}\right]$. Then, it is easy to see that

$$
\mathcal{D}_{\mathrm{EV}}=\mathbb{Z}\left[\tau^{-1}\right] \oplus \underbrace{\varepsilon \mathbb{F}_{2}\left[\varepsilon, \tau, \tau^{-1}\right] \oplus M}_{\mathcal{D}_{\mathrm{EV}}+},
$$

with $M$ as in (17) and that the ring structure has $\mathbb{Z}\left[\tau^{-1}\right] \oplus \varepsilon \mathbb{F}_{2}\left[\varepsilon, \tau, \tau^{-1}\right]$ as a subring and satisfies

$$
\underbrace{\left(\tau^{-1} \mathbb{Z}\left[\tau^{-1}\right] \oplus \mathcal{D}_{\left.\mathrm{EV}_{+}\right)}\right)}_{\widetilde{\mathcal{D}}_{\mathrm{EV}}} \cdot M=\{0\},
$$

where $\widetilde{\mathcal{D}}_{\mathrm{EV}}$ denotes the kernel of the augmentation $\mathcal{D}_{\mathrm{EV}} \rightarrow \mathbb{Z}$.

Remark 2.11 Just as in Remark 2.10(b), we introduce the ideal

$$
J_{\mathrm{EV}}:=\varepsilon \mathbb{F}_{2}\left[\varepsilon, \tau, \tau^{-1}\right] \subset \mathcal{D}_{\mathrm{EV}}
$$

2.6.5 The $\mathcal{B}$-algebra $\mathbb{Z}\left[\xi, \xi^{-1}\right]$ and the operation of smashing with $\mathfrak{S}_{+}$The diagram (11) in the case of $X=$ pt yields an isomorphism of $\mathcal{B}$-modules $H_{\mathrm{Br}}^{*, \bullet}(\mathfrak{S} ; \underline{\mathbb{Z}}) \cong$ $\mathbb{Z}\left[\xi, \xi^{-1}\right]$. Under this isomorphism, the $\mathcal{B}$-module structure of $\mathbb{Z}\left[\xi, \xi^{-1}\right]$ is given by the forgetful functor $\varphi: \mathcal{B} \rightarrow \mathbb{Z}\left[\xi, \xi^{-1}\right]$ and therefore it is determined by the fact that $\tau \in \mathcal{B}^{0,2}$ and $\alpha \in \mathcal{B}^{0,-2}$ act as multiplication by $\xi^{2}$ and $2 \xi^{-2}$, respectively.

More generally, we see from diagram (11) that for any $\mathfrak{S}$-space $X$ there is an isomorphism of $\mathcal{B}$-modules

$$
\gamma^{-1} \circ \varphi: H_{\mathrm{Br}}^{*, \bullet}(X \times \mathfrak{S} ; \underline{\mathbb{Z}}) \rightarrow H_{\text {sing }}^{*}(X ; \mathbb{Z}(\bullet)) \cong H_{\text {sing }}^{*}(X ; \mathbb{Z}) \otimes \mathbb{Z}\left[\xi, \xi^{-1}\right],
$$

where the elements in $H_{\text {sing }}^{*}(X ; \mathbb{Z})$ are given degree $(r, 0)$. The $\mathcal{B}$-algebra structure is determined by the $\mathbb{Z}\left[\xi, \xi^{-1}\right]$-algebra structure of $\left.\mathbb{Z}\left[\xi, \xi^{-1}\right] \otimes H_{\text {sing }}^{*}(X ; \mathbb{Z})\right)$ and by the $\mathcal{B}$-module structure on $\mathbb{Z}\left[\xi, \xi^{-1}\right]$ defined above.

One has a version of diagram (11) for based spaces, with $X \times \mathfrak{S}$ replaced by $X \wedge \mathfrak{S}_{+}$, which yields the following result. 
Lemma 2.12 Let $X$ be a based space on which $\mathfrak{S}$ acts trivially. Then one has an isomorphism of bigraded (nonunital) $\mathcal{B}$-algebras

$$
\tilde{H}_{B r}^{*, *}\left(X \wedge \mathfrak{S}_{+}, \underline{\mathbb{Z}}\right) \cong \tilde{H}_{\text {sing }}^{*}(X ; \mathbb{Z}) \otimes \mathbb{Z}\left[\xi, \xi^{-1}\right],
$$

where the elements in $\tilde{H}_{\text {sing }}^{r}(X ; \mathbb{Z})$ are given degree $(r, 0)$. The structure of the $\mathcal{B}-$ algebra is determined by the $\mathbb{Z}\left[\xi, \xi^{-1}\right]$-algebra structure of $\tilde{H}_{\text {sing }}^{*}(X ; \mathbb{Z}) \otimes \mathbb{Z}\left[\xi, \xi^{-1}\right]$ and by the $\mathcal{B}$-module structure on $\mathbb{Z}\left[\xi, \xi^{-1}\right]$ defined above.

Notation 2.13 Given an $m$-tuple of variables $\boldsymbol{u}=\left(u_{1}, \ldots, u_{m}\right)$ we will denote by $\mathbb{Z}\left[\xi, \xi^{-1}\right](\boldsymbol{u}):=\mathbb{Z}\left[\xi, \xi^{-1}\right]\left(u_{1}, \ldots, u_{m}\right)$ the free bigraded $\mathbb{Z}\left[\xi, \xi^{-1}\right]$-module with generators $u_{i}, i=1, \ldots, m$ in degrees $\operatorname{deg} u_{i}=(1,0)$. Since $\mathbb{Z}\left[\xi, \xi^{-1}\right]$ has a $\mathcal{B}-$ module structure given by the isomorphism $\mathbb{Z}\left[\xi, \xi^{-1}\right] \cong H_{\mathrm{Br}}^{*, \bullet}(\mathfrak{S} ; \underline{\mathbb{Z}})$ then $\mathbb{Z}\left[\xi, \xi^{-1}\right](\boldsymbol{u})$ inherits a $\mathcal{B}$-module structure, which will always be implicit henceforth.

\section{Bredon cohomology of curves of genus 0 and 1}

\subsection{Genus 0}

There are two real curves of genus zero, the projective line $\mathbb{P}_{\mathbb{R}}^{1}$ and the Brauer-Severi curve $B$, whose algebraic structures are completely determined by their respective topological types $\Sigma_{0,1}^{1}$ and $\Sigma_{0,0}^{0}$. As $\mathfrak{S}$-spaces one can identify $\mathbb{P}_{\mathbb{R}}^{1}(\mathbb{C})$ with the representation sphere $S^{2,1}$ and identify $B(\mathbb{C})$ with the sphere $S_{a}^{2}$ (see Notation 2.6). Their Bredon cohomology rings are as follows.

Proposition 3.1 [10, Section 1] One has an isomorphism of $\mathcal{B}$-algebras

$$
H_{\mathrm{Br}}^{*, \bullet}\left(\mathbb{P}_{\mathbb{R}}^{1} ; \underline{\mathbb{Z}}\right) \cong \mathcal{B}[\eta] /\left\langle\eta^{2}\right\rangle,
$$

where $\eta=c_{1}(\mathcal{O}(1)) \in H_{\mathrm{Br}}^{2,1}\left(\mathbb{P}_{\mathbb{R}}^{1} ; \underline{\mathbb{Z}}\right)$ is the first Chern class of the hyperplane bundle $\mathcal{O}(1)$. Similarly, one has an isomorphism of $\mathcal{A}$-algebras (see (16))

$$
H_{\mathrm{Br}}^{*, \bullet}(B ; \underline{\mathbb{Z}}) \cong \mathcal{A}[\eta] /\left\langle\varepsilon^{3}, \varepsilon \eta, \eta^{2}\right\rangle,
$$

where $\eta \in H_{\mathrm{Br}}^{2,1}(B ; \underline{\mathbb{Z}})$ is the first Chern class of $\mathcal{O}(2)$.

Remark 3.2 As bigraded $\mathcal{B}$-modules, one has

$$
\begin{gathered}
H_{\mathrm{Br}}^{*, \bullet}\left(\mathbb{P}_{\mathbb{R}}^{1} ; \underline{\mathbb{Z}}\right) \cong \mathcal{B} \cdot \mathbf{1} \oplus \mathcal{B} \cdot \eta \\
H_{\mathrm{Br}}^{*, \bullet}\left(B ; \underline{\mathbb{Z}} \cong \mathcal{A} /\left\langle\varepsilon^{3}\right\rangle \oplus \mathcal{A} /\langle\varepsilon\rangle \cdot \eta \cong \mathcal{A} /\left\langle\varepsilon^{3}\right\rangle \oplus \mathbb{Z}\left[\tau, \tau^{-1}\right] \cdot \eta .\right.
\end{gathered}
$$




\subsection{Genus 1}

There are three possible types: $\Sigma_{1,2}^{1}, \Sigma_{1,1}^{0}$ and $\Sigma_{1,0}^{0}$.

If $\Sigma(\mathbb{R}) \neq \varnothing$ we choose a base point $p_{0} \in \Sigma(\mathbb{R})$, giving $\Sigma$ the structure of a real elliptic curve with a $\mathfrak{S}$-equivariant isomorphism $\Sigma(\mathbb{C}) \cong \mathbb{C} / \Lambda_{\zeta}$, where $\Lambda_{\zeta}$ is the lattice $\mathbb{Z} \cdot \mathbf{1}+\mathbb{Z} \cdot \zeta$ with $\zeta$ a point in the upper half-plane satisfying $\Re(\zeta) \in\left\{0, \frac{1}{2}\right\}$, and the real structure $\sigma: \mathbb{C} / \Lambda_{\zeta} \rightarrow \mathbb{C} / \Lambda_{\zeta}$ is induced by complex conjugation; see Silhol [13]. The case $\Sigma_{1,2}^{1}$ corresponds to $\zeta=i y$, and the case $\Sigma_{1,1}^{0}$ to $\zeta=\frac{1}{2}+\boldsymbol{i} y$.

3.2.1 Real elliptic curves of separating type $\Sigma_{\mathbf{1 , 2}}^{\mathbf{1}}$ In this case, we have that the torus $\Sigma_{1,2}^{1}(\mathbb{C}) \equiv \mathbb{C} / \Lambda_{\zeta}$ is equivariantly homeomorphic to $S^{1,1} \times S^{1,0}$, according to the topological classification in Section 3.2. Hence $\Sigma_{1,2}^{1}(\mathbb{R}) \cong\{0, \infty\} \times S^{1,0}$ with base point $p_{0}=(\infty, \infty)$. A simple computation gives $H_{\mathrm{Br}}^{*, *}\left(S^{1,1}\right) \cong \mathcal{B}[\mathrm{X}] /\left\langle\mathrm{X}^{2}-\varepsilon \mathrm{X}\right\rangle$, where $\operatorname{deg} \mathrm{X}=(1,1)$, and $H_{\mathrm{Br}}^{*, *}\left(S^{1,0} ; \underline{\mathbb{Z}}\right) \cong \mathcal{B}[\mathrm{Y}] /\left\langle\mathrm{Y}^{2}\right\rangle$, where $\operatorname{deg} \mathrm{Y}=(1,0)$. It is easy to see that the Künneth formula holds in this case yielding the following result.

Proposition 3.3 As a module over $\mathcal{B}$ one has an isomorphism

$$
H_{\mathrm{Br}}^{*, *}\left(\Sigma_{1,2}^{1}(\mathbb{C}) ; \underline{\mathbb{Z}}\right) \cong \mathcal{B} \cdot \mathbf{1} \oplus \mathcal{B}(a, b) \oplus \mathcal{B} \cdot \eta .
$$

The ring structure is given by

$$
H_{\mathrm{Br}}^{*, \bullet}\left(\Sigma_{1,2}^{1}(\mathbb{C}) ; \underline{\mathbb{Z}}\right) \cong \mathcal{B}[a, b, \eta] /\left\langle a^{2}-\varepsilon a, b^{2}, \eta-a b\right\rangle,
$$

where $a$ has degree $(1,1)$ and is Poincaré dual to $\left[\{\infty\} \times S^{1,0}\right]$, while $b$ has degree $(1,0)$ and is dual to $\left[S^{1,1} \times\{\infty\}\right]$ and $\eta$ has degree $(2,1)$. Denoting by $\left\{\alpha_{1}, \beta_{1}\right\}$ the singular homology basis $\left\{\left[\{\infty\} \times S^{1,0}\right],\left[S^{1,1} \times\{\infty\}\right]\right\}$, the images of $a$ and $b$ under the forgetful functor $\varphi$ are expressed in terms of $\left\{\vartheta_{\alpha_{1}}, \vartheta_{\beta_{1}}\right\}$ in Table 6 .

3.2.2 Real elliptic curves of nonseparating type $\Sigma_{\mathbf{1 , 1}}^{\mathbf{0}}$ Once again we represent

$$
\Sigma(\mathbb{C})=\Sigma_{1,1}^{0}(\mathbb{C})=\mathbb{C} / \Lambda_{\zeta} \quad \text { with } \quad \zeta=\frac{1}{2}+i y .
$$

For simplicity, we write $\Sigma=\Sigma(\mathbb{C})$ in what follows. Let $D$ be a small open disc around $\frac{1}{2}$ in $\mathbb{C}$, contained in the interior of the convex hull of $\{0,1, \zeta, \bar{\zeta}\}$. See Figure 4. If $\pi: \Sigma \rightarrow \Sigma /(\Sigma-D) \cong S^{2,1}$ is the quotient map, we obtain two maps

$$
H_{\mathrm{Br}}^{*, \bullet}(\Sigma ; \underline{\mathbb{Z}})_{\pi^{*}}^{\stackrel{\pi_{\dagger}}{\longleftarrow}} H_{\mathrm{Br}}^{*, \bullet}\left(S^{2,1} ; \underline{\mathbb{Z}}\right),
$$

where $\pi_{\dagger}$ is the $\mathcal{B}$-module map obtained as an umker map. In other words, given $\alpha \in H_{\mathrm{Br}}^{*, \bullet}(\Sigma ; \underline{\mathbb{Z}})$ one has $\pi_{\dagger}(\alpha) \cap\left[S^{2,1}\right]=\pi_{*}(\alpha \cap[\Sigma])$. It is easy to see $\pi_{\dagger} \circ \pi^{*}=\mathrm{Id}$, 
and hence $H_{\mathrm{Br}}^{*, \bullet}\left(S^{2,1} ; \underline{\mathbb{Z}}\right)$ becomes a subring of $H_{\mathrm{Br}}^{*, \bullet}(\Sigma ; \underline{\mathbb{Z}})$, split as a $\mathcal{B}$-submodule. The long exact cohomology sequence for the pair $(\Sigma, \Sigma-D)$ splits, giving

$$
H_{\mathrm{Br}}^{*, \bullet}(\Sigma ; \underline{\mathbb{Z}}) \cong H_{\mathrm{Br}}^{*, \bullet}\left(S^{2,1} ; \underline{\mathbb{Z}}\right) \oplus \tilde{H}_{\mathrm{Br}}^{*, \bullet}(\Sigma-D ; \underline{\mathbb{Z}}) .
$$

On the other hand, the canonical radial deformation away from the center of $D$ gives an equivariant homotopy equivalence $\Sigma-D \simeq S^{1,0} \wedge \mathfrak{S}_{+}$, and hence $\widetilde{H}_{\mathrm{Br}}^{*, \bullet}(\Sigma-D ; \underline{\mathbb{Z}}) \cong$ $\mathbb{Z}\left[\xi, \xi^{-1}\right](u)$, where $\operatorname{deg} u=(1,0)$. This gives an isomorphism of $\mathcal{B}$-modules

$$
H_{\mathrm{Br}}^{*, \bullet}(\Sigma ; \underline{Z}) \cong \mathcal{B} \cdot \mathbf{1} \oplus \mathbb{Z}\left[\xi, \xi^{-1}\right](u) \oplus \mathcal{B} \cdot \eta,
$$

where $\eta$ has degree $(2,1)$ and is the Poincaré dual of $\left[p_{0}\right]$.

In the presentation (23) the classes $\xi^{r} u$ are unique up to a sign. We will fix this choice by defining $u$ as the Poincaré dual of a geometric generator for the homology group $H_{1,1}^{\mathrm{Br}}(\Sigma ; \underline{\mathbb{Z}})$, which we present next. We will also present the generators for $H_{1,2}^{\mathrm{Br}}(\Sigma ; \underline{\mathbb{Z}})$ and $H_{1,3}^{\mathrm{Br}}(\Sigma ; \underline{\mathbb{Z}})$ needed to complete the presentation of the ring structure in $H_{\mathrm{Br}}^{*, \bullet}(\Sigma ; \underline{\mathbb{Z}})$. These homology classes will be obtained from three equivariant maps

$$
J: S^{1,0} \rightarrow \Sigma, \quad \pi: S^{1,1} \rightarrow \Sigma \quad \text { and } \quad \rho: S_{a}^{1} \rightarrow \Sigma,
$$

described as follows. First, let

$$
J: S^{1,0} \hookrightarrow \Sigma
$$

denote the inclusion of the fixed point set. This is represented by the intersection of the real line with the convex hull of $\{0,1, \zeta, \bar{\zeta}\}$, the chosen fundamental domain for the action of $\Lambda_{\zeta}$ on $\mathbb{C}$. Similarly, let

$$
l: S^{1,0} \wedge \mathfrak{S}_{+} \hookrightarrow \Sigma
$$

denote the inclusion of the curve represented by the intersection of the upper half-plane with the boundary of the fundamental domain.

It is easy to construct an equivariant projection $\hat{\pi}: S^{1,1} \rightarrow S^{1,0} \wedge \mathfrak{S}_{+}$which is bijective on the complement of the fixed point set of the $\mathfrak{S}$-action, and such that $\hat{\pi}_{*}\left[S^{1,1}\right]$ is a generator of $H_{1,1}^{\mathrm{Br}}\left(S^{1,0} \wedge \mathfrak{S}_{+} ; \underline{\mathbb{Z}}\right)$. Then define $\pi$ as the composition:

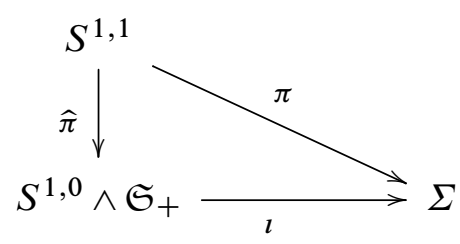

Finally, we also have an equivariant inclusion $\rho: S_{a}^{1} \hookrightarrow \Sigma$ represented by the intersection of the fundamental domain with the horizontal lines passing through $\zeta / 2$ and $\bar{\zeta} / 2$, 
respectively. Using the same radial deformation as above one obtains an equivariantly homotopy-commutative diagram:

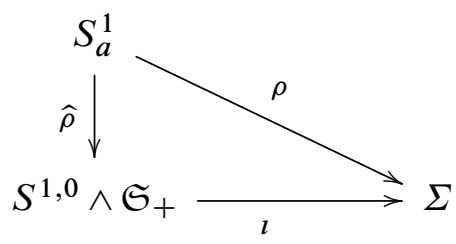

Lemma 3.4 Let $\left[S^{1,0}\right]$, $\left[S^{1,1}\right]$ and $\left[S_{a}^{1}\right]$ denote the fundamental homology classes, of degrees $(1,0),(1,1)$ and $(1,2)$, respectively (see Costenoble and Waner [2] and [11, page 1264]). Then the Poincare dual of $\pi_{*}\left[S^{1,1}\right]$ is a generator $u \in H_{\mathrm{Br}}^{1,0}(\Sigma ; \underline{\mathbb{Z}})$. Furthermore, the Poincaré duals of $j_{*}\left[S^{1,0}\right]$ and $\rho_{*}\left[S_{a}^{1}\right]$ are $\xi u \in H_{\mathrm{Br}}^{1,1}(\Sigma ; \underline{\mathbb{Z}})$ and $\xi^{-1} u \in H_{\mathrm{Br}}^{1,-1}(\Sigma ; \underline{Z})$, respectively.

Proof This follows easily from an application of corresponding forgetful functors and the $\mathcal{B}$-module structure of $H_{\mathrm{Br}}^{*, \bullet}(\Sigma ; \underline{\mathbb{Z}})$ given in (23).

Remark 3.5 From the definition of $\pi, l$ and $\rho$ one sees that the real basis $\left\{\alpha_{1}, \beta_{1}\right\}$ of $H_{1}^{\text {sing }}(\Sigma ; \mathbb{Z})$ in Figure 4 has the following relation to the Bredon homology of $\Sigma$ under the forgetful functor:

$$
\varphi\left(j_{*}\left[S^{1,0}\right]\right)=\alpha_{1} \quad \text { and } \quad \varphi\left(\pi_{*}\left(\left[S^{1,1}\right]\right)\right)=2 \pi \boldsymbol{i}\left(\beta_{1}-\sigma_{*} \beta_{1}\right) .
$$

Proposition 3.6 As a module over $\mathcal{B}$ one has a direct sum decomposition

$$
H_{\mathrm{Br}}^{*, \bullet}\left(\Sigma_{1,1}^{0}(\mathbb{C}) ; \underline{\mathbb{Z}}\right)=\mathcal{B} \cdot \mathbf{1} \oplus \mathbb{Z}\left[\xi, \xi^{-1}\right](u) \oplus \mathcal{B} \cdot \eta,
$$

where $u$ is a generator of degree $(1,0)$. The product structure is determined by the relation

$$
\xi^{k} u \cdot \xi^{l} u=(-1)^{k} \Gamma_{k+l}, \quad \text { where } \quad \Gamma_{s}=\left\{\begin{array}{ll}
\tau^{(s+1) / 2} \alpha \eta & s \text { odd } \\
0 & \text { s even }
\end{array} \text { in } \mathcal{B}^{0, s-1} .\right.
$$

The image of $u$ and $\xi u$ under the forgetful functor is expressed in terms of the symplectic basis $\left\{\vartheta_{\alpha_{1}}, \vartheta_{\beta_{1}}\right\}$ in Table 6 .

Proof The $\mathcal{B}$-module structure was described in (23), using an inclusion of $\mathcal{B} \cdot \mathbf{1} \oplus$ $\mathcal{B} \cdot \eta \equiv H_{\mathrm{Br}}^{*, \bullet}\left(S^{2,1} ; \underline{\mathbb{Z}}\right)$ into $H_{\mathrm{Br}}^{*, \bullet}(\Sigma ; \underline{\mathbb{Z}})$ as a $\mathcal{B}$-subalgebra. In particular, $\eta^{2}=0$.

Next we establish the identities

$$
u \cdot\left(\xi^{-1} u\right)=\alpha \eta, \quad u \cdot\left(\xi^{-2}\right) u=0, \quad\left(\xi^{-1} u\right) \cdot\left(\xi^{-1} u\right)=0 .
$$


We know that $u \cdot\left(\xi^{-1} u\right)=k \alpha \eta$, for some integer $k$. On the other hand, it follows from the construction of $\pi: S^{1,1} \rightarrow \Sigma$, Lemma 3.4 and an application of the forgetful functor that $u \cdot(\xi u)=2 \eta$. Using the $\mathcal{B}$-module structure of $\mathbb{Z}\left[\xi, \xi^{-1}\right]$ one gets $2 \eta=u \cdot(\xi u)=\tau\left(u \cdot\left(\xi^{-1} u\right)\right)=\tau(k \alpha \eta)=2 k \eta$ and, since $H_{\mathrm{Br}}^{2,1}(\Sigma ; \underline{\mathbb{Z}})$ is a free abelian group generated by $\eta$, one concludes that $k=1$.

Using Lemma 3.4 and the projection formula, one obtains that $\left\{u \cdot\left(\xi^{-2} u\right)\right\} \cap[\Sigma]=$ $\left(\xi^{-2} u\right) \cap(u \cap[\Sigma])=\left(\xi^{-2} u\right) \cap \pi_{*}\left[S^{1,1}\right]=\pi_{*}\left(\pi^{*}\left(\xi^{-2} u\right) \cap\left[S^{1,1}\right]\right)$. Observe that $\pi^{*}\left(\xi^{-2} u\right) \cap\left[S^{1,1}\right]$ lies in the torsion group $H_{0,3}^{\mathrm{Br}}\left(S^{1,1} ; \underline{\mathbb{Z}}\right) \cong H_{\mathrm{Br}}^{1,-2}\left(S^{1,1} ; \underline{\mathbb{Z}}\right)$. However, since $\pi: S^{1,1} \rightarrow \Sigma$ factors through $S^{1,0} \wedge \mathfrak{S}_{+}$as described in (26), and the Bredon homology of $S^{1,0} \wedge \mathfrak{S}_{+}$is free, one concludes that $\pi_{*}\left(\pi^{*}\left(\xi^{-2} u\right) \cap\left[S^{1,1}\right]\right)=0$.

A similar argument, using (27), Lemma 3.4 and the fact that $H_{0,3}^{\mathrm{Br}}\left(S_{a}^{1} ; \underline{\mathbb{Z}}\right) \cong H_{\mathrm{Br}}^{1,1}\left(S_{a}^{1} ; \underline{\mathbb{Z}}\right)$ is torsion [10, page 4711] shows that $\left(\xi^{-1} u\right)\left(\xi^{-1} u\right)=0$.

Now it is easy to check that

$\left(\xi^{2 k} u\right) \cdot\left(\xi^{2 l} u\right)=0, \quad\left(\xi^{2 k} u\right) \cdot\left(\xi^{-1+2 l} u\right)=\left(\tau^{k+l} \alpha\right) \eta, \quad\left(\xi^{-1+2 k} u\right) \cdot\left(\xi^{-1+2 l} u\right)=0$

for all $k, l \in \mathbb{Z}$. Indeed, the second identity follows immediately from an application of the forgetful functor, which gives an isomorphism $H_{\mathrm{Br}}^{2,2(k+l)-1}(\Sigma ; \underline{\mathbb{Z}}) \rightarrow$ $H_{\text {sing }}^{2}(\Sigma ; \mathbb{Z}(2(k+l)-1))^{\mathfrak{S}} \cong \mathbb{Z}$.

We prove the first identity. Since $\tau \cdot\left(\xi^{2 k} u\right) \cdot\left(\xi^{2 l} u\right)=\left(\xi^{2 k+2} u\right) \cdot\left(\xi^{2 l} u\right)=\left(\xi^{2 k} u\right)$. $\left(\xi^{2 l+2} u\right)$, it suffices to show that $u \cdot \xi^{2 l} u=0$ if $l<-1$. From the additive structure of $H_{\mathrm{Br}}^{*, \bullet}(\Sigma(\mathbb{C}) ; \underline{Z})$ it follows that $u \cdot \xi^{2 l} u$ is multiple of $\tau^{l+1} \theta \cdot \eta$. But $\tau^{-l-1} \cdot\left(u \cdot \xi^{2 l} u\right)=$ $\left(u \cdot \xi^{-2} u\right)=0$, and $\tau^{-l-1} \tau^{l+1} \theta \cdot \eta=\theta \cdot \eta$ hence $u \cdot \xi^{2 l} u=0$.

The last identity follows in a similar fashion. This concludes the proof.

3.2.3 Genus 1 curves without real points $\Sigma_{\mathbf{1}, 0}^{\mathbf{0}}$ A choice of $p_{0} \in \Sigma_{1,0}^{0}(\mathbb{C})$ gives an isomorphism of real curves $\Sigma_{1,0}^{0}(\mathbb{C}) \cong \mathbb{C} / \Lambda_{\zeta}$, where $\Lambda_{\zeta}=\mathbb{Z}+\mathbb{Z} \cdot \zeta$ and $\zeta$ is a point in the upper half-plane which satisfies $\Re \zeta=0$, and the real structure on $\mathbb{C} / \Lambda_{\zeta}$ is induced by $\sigma_{\lambda}: z \mapsto \bar{z}+\lambda$, for some $\lambda \in \frac{1}{2} \Lambda_{\zeta}$; see [5; 13]. We can assume that $\lambda=\frac{1}{2}$.

Consider the basis $\{C, D\}$ for $H_{1}^{\text {sing }}\left(\Sigma_{1,0}^{0}(\mathbb{C}) ; \mathbb{Z}\right)$, where $C$ is determined by the line through $\frac{1}{2}$ perpendicular to the real axis, oriented as in Figure 5 and $D$ is the circle in $\Sigma_{1,0}^{0}(\mathbb{C})$ determined by the real axis in $\mathbb{C}$.

Proposition 3.7 There is an equivariant homeomorphism $\Sigma_{1,0}^{0}(\mathbb{C}) \cong S^{1,1} \times S_{a}^{1}$. Under this isomorphism $\{\infty\} \times S_{a}^{1}$ and $S^{1,1} \times S_{a}^{0}$ are identified with $D$ and $C \amalg\left(\frac{1}{2}+C\right)$ as in Figure 5. There is a ring isomorphism

$$
H_{\mathrm{Br}}^{*, \bullet}\left(\Sigma_{1,0}^{0}(\mathbb{C}) ; \underline{\mathbb{Z}}\right) \cong \mathcal{A}[\mathrm{s}, \mathrm{T}, \eta] /\left\langle\mathrm{s}^{2}-\varepsilon \mathrm{S}, \varepsilon^{2}, \varepsilon \mathrm{T}, \mathrm{T}^{2}, \eta-\mathrm{ST}\right\rangle,
$$


where $\mathrm{S}$ has degree $(1,1)$ and is Poincaré dual to $-\tau \cap\left[\infty \times S_{a}^{1}\right]$, while $\mathrm{T}$ has degree $(1,0)$ and is dual to $\left[S_{a}^{0} \times S^{1,1}\right]$ and $\eta$ has degree $(2,1)$. As $\mathcal{A}$-module this gives

$$
H_{\mathrm{Br}}^{*, \bullet}\left(\Sigma_{1,0}^{0}(\mathbb{C}) ; \underline{\mathbb{Z}}\right) \cong \mathcal{A} /\left\langle\varepsilon^{2}\right\rangle \oplus \mathcal{A} /\left\langle\varepsilon^{2}\right\rangle \cdot \mathrm{S} \oplus \mathbb{Z}\left[\tau, \tau^{-1}\right] \cdot \mathrm{T} \oplus \mathbb{Z}\left[\tau, \tau^{-1}\right] \cdot \eta .
$$

The image of $\mathrm{S}$ and $\mathrm{T}$ under the forgetful functor is expressed in terms of the symplectic basis $\left\{\vartheta_{\alpha_{1}}, \vartheta_{\beta_{1}}\right\}$ in Table 6 , where $\alpha_{1}=-D$ and $\beta_{1}=C$.

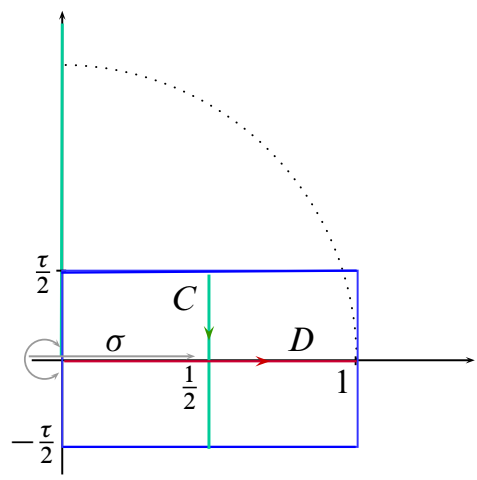

Figure 5: Genus 1 curve without real points $\Sigma_{1,0}^{0}$; the action is given by $\sigma(z)=\bar{z}+\frac{1}{2}$.

Proof The first two statements are clear from Figure 5. Since the cohomology of $S^{1,1}$ is free as a module over $\mathcal{B}$, the Künneth formula together with the computation

$$
H_{\mathrm{Br}}^{*, \bullet}\left(S_{a}^{1} ; \underline{\mathbb{Z}}\right) \cong \mathcal{A}[\mathrm{T}] /\left\langle\varepsilon^{2}, \varepsilon \mathrm{T}, \mathrm{T}^{2}\right\rangle, \quad \operatorname{deg} \mathrm{T}=(1,0)
$$

in [10, page 4711] yields the ring isomorphism in the statement.

The assertions concerning the Poincaré duals of $\mathrm{S}$ and $\mathrm{T}$ follow from the $\mathcal{A}$-module structure of $H_{\mathrm{Br}}^{*, \bullet}\left(\Sigma_{1,0}^{0}(\mathbb{C}) ; \underline{\mathbb{Z}}\right)$ and nonequivariant Poincaré duality (note $\left[\{\infty\} \times S_{a}^{1}\right]$ has degree $(1,2)$; see [11, page 1264$])$.

\section{The bigraded Bredon cohomology ring of arbitrary real curves}

The goal of this section is to establish the following result.

Theorem 4.1 Let $\Sigma$ be a real curve, and consider sets of variables $\boldsymbol{a}:=\left\{a_{1}, \ldots, a_{r-1}\right\}$, $\boldsymbol{b}=\left\{b_{1}, \ldots, b_{r-1}\right\}, \boldsymbol{u}=\left\{u_{1}, \ldots, u_{k}\right\}$, where $\operatorname{deg} a_{i}=\operatorname{deg} \xi u_{j}=(1,1), \operatorname{deg} b_{i}=$ $\operatorname{deg} u_{j}=(1,0)$, while $r$ and $k$ are indicated in each case below, and a variable $\eta$ with $\operatorname{deg} \eta=(2,1)$. 
(I) If $\Sigma=\Sigma_{g, r}^{1}$ then $g+1=r+2 q$ with $q \in \mathbb{N} \cup\{0\}$ and the $\mathcal{B}$-module structure of $H_{\mathrm{Br}}^{*, \bullet}(\Sigma ; \underline{Z})$ is determined by

$$
H_{\mathrm{Br}}^{*, \bullet}(\Sigma(\mathbb{C}) ; \underline{\mathbb{Z}}) \cong \mathcal{B} \cdot \mathbf{1} \oplus \mathcal{B}(\boldsymbol{a}, \boldsymbol{b}) \oplus \mathbb{Z}\left[\xi, \xi^{-1}\right](\boldsymbol{u}) \oplus \mathcal{B} \cdot \eta,
$$

with $\boldsymbol{u}=\left\{u_{1}, \ldots, u_{2 q}\right\}$. The ring structure is determined by the relations

(1) $a_{i} \cdot a_{j}=\delta_{i j} \varepsilon a_{i}$,

(2) $b_{i} \cdot b_{j}=0$,

(3) $a_{i} \cdot b_{j}=\delta_{i j} \eta$,

(4) $x \cdot y=0$ for all $x \in \mathcal{B}(\boldsymbol{a}, \boldsymbol{b}), y \in \mathbb{Z}\left[\xi, \xi^{-1}\right](\boldsymbol{u})$,

(a) $\xi^{k} u_{i} \cdot \xi^{l} u_{j}=(-1)^{k} \delta_{i+j, 2 q+1} \Gamma_{k+l}^{\Sigma}$,

where $\Gamma_{k+l}^{\Sigma} \in H_{\mathrm{Br}}^{2, k+l}(\Sigma(\mathbb{C}) ; \underline{\mathbb{Z}})$ is given in Table 5 .

\begin{tabular}{ccc}
$\Sigma$ & \multicolumn{2}{c}{$\Gamma_{s}^{\Sigma}$} \\
\cline { 2 - 3 } & $s$ odd & $s$ even \\
\hline$\Sigma_{g, r}^{1}$ & $\tau^{(s+1) / 2} \alpha \eta$ & $\tau^{(s+2) / 2} \theta \eta$ \\
$\Sigma_{g, r}^{0}$ & $\tau^{(s+1) / 2} \alpha \eta$ & 0 \\
$\Sigma_{2 q, 0}^{0}$ & $\tau^{(s-1) / 2} \eta$ & $\tau^{(s-2) / 2} \varepsilon^{2}$ \\
$\Sigma_{2 q+1,0}^{0}$ & $\tau^{(s-1) / 2} \eta$ & $\tau^{(s-2) / 2} \varepsilon S$
\end{tabular}

Table 5: Values of $\Gamma_{s}^{\Sigma}$

(II) If $\Sigma=\Sigma_{g, r}^{0}$ with $r \geq 1$, then the $\mathcal{B}$-module structure of $H_{\mathrm{Br}}^{*, \bullet}(\Sigma ; \underline{\mathbb{Z}})$ is given by

$$
H_{\mathrm{Br}}^{*, \bullet}(\Sigma(\mathbb{C}) ; \underline{\mathbb{Z}}) \cong \mathcal{B} \cdot \mathbf{1} \oplus \mathcal{B}(\boldsymbol{a}, \boldsymbol{b}) \oplus \mathbb{Z}\left[\xi, \xi^{-1}\right](\boldsymbol{u}) \oplus \mathcal{B} \cdot \eta,
$$

with $\boldsymbol{u}=\left\{u_{1}, \ldots, u_{g+1-r}\right\}$. The relations (1)-(4) together with

(a) $\xi^{k} u_{i} \cdot \xi^{l} u_{j}=(-1)^{k} \delta_{i j} \Gamma_{k+l}^{\Sigma}$,

determine the ring structure.

(III) If $\Sigma=\Sigma_{g, 0}^{0}$ with $g=2 q$, then the $\mathcal{B}$-module structure of $H_{\mathrm{Br}}^{*, \bullet}(\Sigma ; \underline{\mathbb{Z}})$ is given by

$$
H_{\mathrm{Br}}^{*, \bullet}(\Sigma(\mathbb{C}) ; \underline{\mathbb{Z}}) \cong \mathcal{A}[\eta] /\left\langle\varepsilon^{3}, \varepsilon \eta, \eta^{2}\right\rangle \oplus \mathbb{Z}\left[\xi, \xi^{-1}\right](\boldsymbol{u})
$$

with $\boldsymbol{u}=\left\{u_{1}, \ldots, u_{2 q}\right\}$. The ring structure is determined by the condition that $\mathcal{A}[\eta] /\left\langle\varepsilon^{3}, \varepsilon \eta, \eta^{2}\right\rangle$ is a subring together with the relation (a). 
(IV) If $\Sigma=\Sigma_{g, 0}^{0}$ with $g=2 q+1$, then the $\mathcal{B}$-module structure of $H_{\mathrm{Br}}^{*, \bullet}(\Sigma ; \underline{\mathbb{Z}})$ is given by

$$
H_{\mathrm{Br}}^{*, \bullet}(\Sigma(\mathbb{C}) ; \underline{\mathbb{Z}}) \cong \mathcal{A}[\mathrm{S}, \mathrm{T}, \eta]\left\langle\mathrm{S}^{2}-\varepsilon \mathrm{S}, \varepsilon^{2}, \varepsilon \mathrm{T}, \mathrm{T}^{2}, \eta-\mathrm{ST}\right\rangle \oplus \mathbb{Z}\left[\xi, \xi^{-1}\right](\boldsymbol{u})
$$

with $\boldsymbol{u}=\left\{u_{1}, \ldots, u_{2 q}\right\}, \operatorname{deg} \mathrm{S}=(1,1)$ and $\operatorname{deg} \mathrm{T}=(1,0)$. The ring structure is determined by the condition that the first summand is a subring, together with the relations $\mathrm{S} \cdot u_{i}=\mathrm{T} \cdot u_{i}=0$ and (s).

Furthermore, the images of the generators of $\boldsymbol{a}, \boldsymbol{b}, \boldsymbol{u}, \xi \boldsymbol{u}, \mathrm{S}$ and $\mathrm{T}$ under the forgetful functor $\varphi$ are expressed in terms of the symplectic basis $\left\{\vartheta_{\boldsymbol{\alpha}}, \vartheta_{\boldsymbol{\beta}}\right\}$ in Table 6 below.

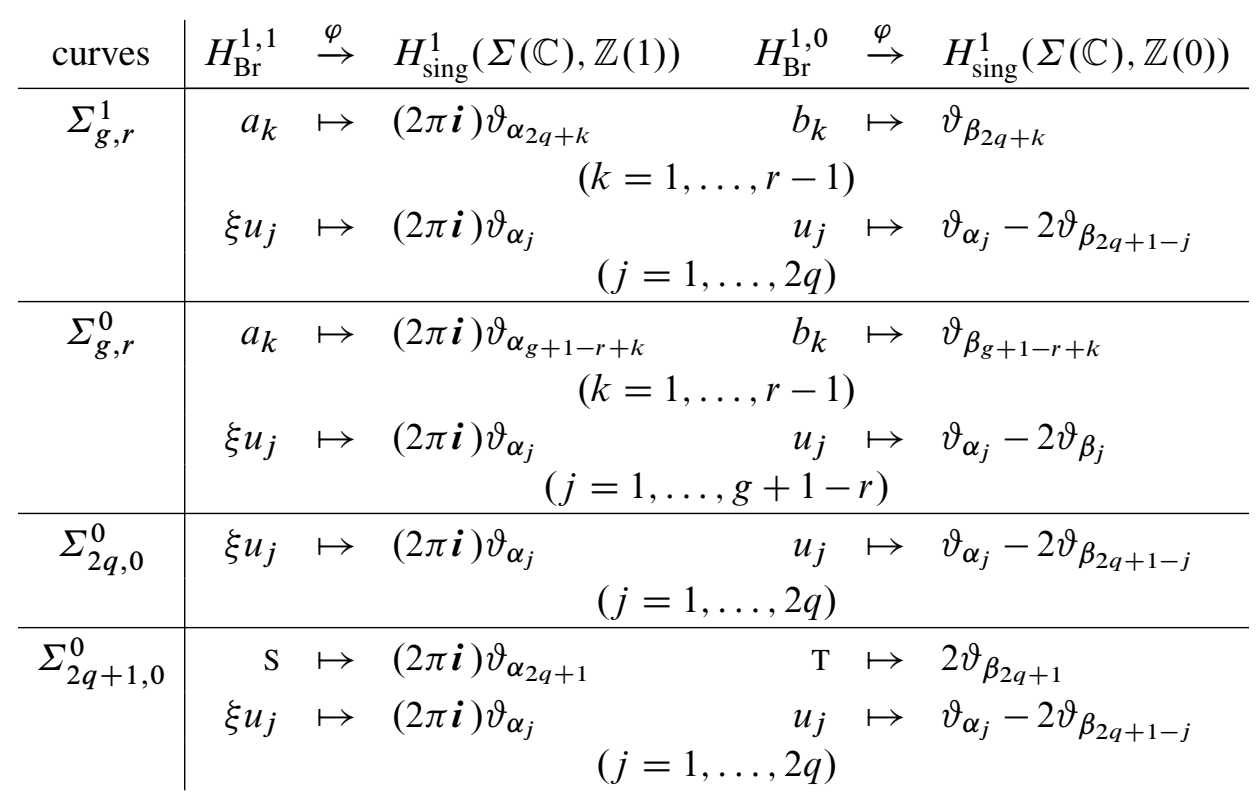

Table 6: Image of the generators of $H_{\mathrm{Br}}^{*, \bullet}\left(\Sigma_{g, r}^{\varepsilon} ; \underline{\mathbb{Z}}\right)$ under the forgetful functor

\subsection{Equivariant connected sums}

4.1.1 Equivariant connected sum 1 Let $x_{0} \in X(\mathbb{R})$ and $y_{0} \in Y(\mathbb{R})$ be real points in two smooth complete real curves $X$ and $Y$ and choose sufficiently small closed disks $D:=D_{x_{0}}$ and $D^{\prime}:=D_{y_{0}}$ centered at $x_{0}$ and $y_{0}$, respectively, together with equivariant diffeomorphisms $D \cong D^{2,1} \cong D^{\prime}$. One can define an equivariant connected sum $X(\mathbb{C}) \#_{\mathrm{D}^{2,1}} Y(\mathbb{C})$ by gluing $X-\stackrel{\bar{\circ}}{D}$ and $Y-\stackrel{\circ}{D}^{\prime}$ along $\partial D$ and $\partial D^{\prime}$, using the given equivariant diffeomorphism. 
Notation 4.2 It is easy to see that if $\Sigma$ and $\Sigma^{\prime}$ are real curves of type $\Sigma_{g, r}^{\varepsilon}$ and $\Sigma_{g^{\prime}, r^{\prime}}^{\varepsilon^{\prime}}$ with $r, r^{\prime}>0$, then $\Sigma \#_{\mathrm{D}^{2,1}} \Sigma^{\prime}$ has type $\Sigma_{g+g^{\prime}, r+r^{\prime}-1}^{\varepsilon \varepsilon^{\prime}}$. To abbreviate this statement we will write

$$
\Sigma_{g+g^{\prime}, r+r^{\prime}-1}^{\varepsilon \varepsilon^{\prime}}=\Sigma_{g, r}^{\varepsilon} \#_{\mathrm{D}^{2,1}} \Sigma_{g^{\prime}, r^{\prime}}^{\varepsilon^{\prime}}
$$

Given a nonunital $\mathcal{B}$-algebra $\widetilde{R}$, one can construct a corresponding unital augmented $\mathcal{B}$-algebra $R:=\mathcal{B} \cdot 1 \oplus \widetilde{R}$ having $\widetilde{R}$ as the kernel of the augmentation $R \rightarrow \mathcal{B} \cdot 1$. In what follows we will often encounter nonunital $\mathcal{B}$-algebras of the form $\widetilde{R}=N_{R} \oplus \mathcal{B} \cdot \eta_{R}$, where $\eta$ has degree $(2,1)$ and $N_{R}$ is a $\mathcal{B}$-module generated by elements of degree $(1, *)$ with $* \in \mathbb{Z}$. Whenever $\widetilde{R}$ and $\widetilde{S}$ are $\mathcal{B}$-algebras of this form, define their connected sum $\widetilde{R} \circledast \widetilde{S}$ as

$$
\widetilde{R} \circledast \widetilde{S}:=(\widetilde{R} \times \widetilde{S}) /\left\langle\eta_{R}-\eta_{S}\right\rangle,
$$

where $\widetilde{R} \times \tilde{S}$ is seen as the bigraded $\mathcal{B}$-module $\widetilde{R} \oplus \widetilde{S}$ and given the product ring structure, and $\left\langle\eta_{R}-\eta_{S}\right\rangle$ is the ideal generated by $\left(\eta_{R}, 0\right)-\left(0, \eta_{S}\right)$. We denote the associated unital $\mathcal{B}$-algebra by $R \circledast S$ and observe that it also has the form

$$
R \circledast S=\mathcal{B} \cdot \mathbf{1} \oplus\left(N_{R} \oplus N_{S}\right) \oplus \eta \cdot \mathcal{B},
$$

where $\eta=\left[\left(\eta_{R}, 0\right)\right]=\left[\left(0, \eta_{S}\right)\right]$, and $[(a, b)]$ represents the class of $(a, b)$ in the quotient.

Lemma 4.3 Given $\left(X, x_{0}\right)$ and $\left(Y, y_{0}\right)$ as above, let $\eta_{X} \in H_{\mathrm{Br}}^{2,1}(X(\mathbb{C}) ; \underline{\mathbb{Z}})$ and $\eta_{Y} \in H_{\mathrm{Br}}^{2,1}(Y(\mathbb{C}) ; \underline{\mathbb{Z}})$ denote the Poincaré duals of $\left[x_{0}\right] \in H_{0,0}(X(\mathbb{C}) ; \underline{\mathbb{Z}})$ and $\left[y_{0}\right] \in H_{0,0}(Y(\mathbb{C}) ; \underline{\mathbb{Z}})$, respectively.

(a) The Bredon cohomology of $X(\mathbb{C})$ can be written as a direct sum of $\mathcal{B}$-modules

$$
H_{\mathrm{Br}}^{*, *}(X(\mathbb{C}) ; \underline{\mathbb{Z}})=\mathcal{B} \cdot \mathbf{1} \oplus N(X(\mathbb{C})) \oplus \mathcal{B} \cdot \eta_{X} .
$$

(b) As a ring, $H_{\mathrm{Br}}^{*, \bullet}\left(X(\mathbb{C}) \sharp_{D^{2,1}} Y(\mathbb{C}) ; \underline{\mathbb{Z}}\right)$ is isomorphic to the "pushout"

$$
H_{\mathrm{Br}}^{*, *}(X(\mathbb{C}) ; \underline{\mathbb{Z}}) \circledast H_{\mathrm{Br}}^{*, *}(Y(\mathbb{C}) ; \underline{\mathbb{Z}})
$$

as defined in (30).

Proof The first assertion follows immediately from splitting off the cohomology of a point using the fixed point $x_{0}$ and Poincaré duality. The second assertion uses the same arguments that one would use to prove the same result for singular cohomology (Mayer-Vietoris sequences, excision, homotopy invariance etc). 
Example 4.4 (Bredon cohomology of curves of type $\Sigma_{r-1, r}^{1}$ ) Let $r \geq 2$. Applying the operation $\#_{\mathrm{D}^{2,1}}$ to glue $r-1$ curves of type $\Sigma_{1,2}^{1}$ we obtain

$$
\Sigma_{r-1, r}^{1}=\underbrace{\Sigma_{1,2}^{1} \#_{\mathrm{D}^{2,1}} \cdots \#_{\mathrm{D}^{2,1}} \Sigma_{1,2}^{1}}_{(r-1) \text { times }} .
$$

Hence, for a curve $\Sigma$ of type $\Sigma_{r-1, r}^{1}$, it follows inductively from Lemma 4.3(a) that there is an isomorphism of $\mathcal{B}$-modules

$$
H_{\mathrm{Br}}^{*, \bullet}(\Sigma(\mathbb{C}) ; \underline{\mathbb{Z}}) \cong \mathcal{B} \cdot \mathbf{1} \oplus \mathcal{B}(\boldsymbol{a}, \boldsymbol{b}) \oplus \mathcal{B} \cdot \eta,
$$

where $(\boldsymbol{a}, \boldsymbol{b})=\left\{a_{1}, \ldots, a_{r-1}, b_{1}, \ldots, b_{r-1}\right\}, \operatorname{deg} a_{i}=(1,1)$ and $\operatorname{deg} b_{i}=(1,0)$. Lemma 4.3(b) implies that the ring structure is determined by the relations (1), (2) and (3) in Theorem 4.1.

It is clear from the geometry of this construction that starting from the symplectic basis $\left\{\alpha_{1}, \beta_{1}\right\} \subset H_{1}^{\operatorname{sing}}\left(\Sigma_{1,2}^{1}(\mathbb{C}) ; \mathbb{Z}\right)$ of Proposition 3.3 one obtains a symplectic basis $\{\boldsymbol{\alpha}, \boldsymbol{\beta}\}$ for $H_{1}^{\text {sing }}\left(\Sigma_{r-1, r}^{1}(\mathbb{C}) ; \mathbb{Z}\right)$ as depicted in Figure 1 . The images of the equivariant cohomology classes $\boldsymbol{a}, \boldsymbol{b}$ under the forgetful functor are expressed in terms of the dual basis $\left\{\vartheta_{\boldsymbol{\alpha}}, \vartheta_{\boldsymbol{\beta}}\right\}$ in Table 6 .

4.1.2 Equivariant connected sum 2 Let $\mathcal{S}$ be a real curve and pick a small open $\operatorname{disc} D \subset \mathcal{S}(\mathbb{C})-\mathcal{S}(\mathbb{R})$ around $x_{0} \in \mathcal{S}(\mathbb{C})$. Denote $\mathcal{S}:=\mathcal{S}-\{D \cup \sigma(D)\}$. Given a complex curve $\mathcal{C}_{q}$ of genus $q$, let $\mathcal{C}_{q}$ be the complement of a small open disk $\widehat{D}$ around $y_{0} \in \mathcal{C}_{q}$. Consider the product $\mathcal{C}_{q} \times \mathfrak{S}$ as the disjoint union

$$
\stackrel{\circ}{\mathcal{C}}_{q} \amalg \stackrel{\bar{\circ}}{\mathcal{C}}_{q}
$$

where $\stackrel{\overline{\mathcal{C}}}{\mathcal{C}}_{q}$ denotes $\stackrel{\circ}{\mathcal{C}}_{q}$ with the opposite orientation, so that the usual action on $\stackrel{\circ}{\mathcal{C}}_{q} \times \mathfrak{S}$ becomes an antiholomorphic involution.

Define $\Sigma$ as a connected sum $\Sigma:=\mathcal{C}_{q} \#_{\partial D} \mathcal{S} \#_{\partial(\sigma * D)} \mathcal{C}_{q}$ which is homeomorphic to the quotient

$$
\Sigma:=\left(\stackrel{\circ}{\mathcal{S}} \amalg \stackrel{\circ}{\mathcal{C}}_{q} \times \mathfrak{S}\right) / \sim,
$$

where $\sim$ is the equivalence relation generated as follows. Fix a diffeomorphism $\varphi: \partial \widehat{D} \rightarrow \partial D$ and identify $x \in \partial D$ to $(\varphi(x), 1))$ and $x \in \partial \sigma(D)$ to $(\varphi(x), \sigma)$. The induced $\mathfrak{S}$-action on $\Sigma$ is an orientation-reversing diffeomorphism $\sigma$, and hence $(\Sigma, \sigma)$ has the equivariant topological type of a real curve — also denoted $\Sigma$ - satisfying $\Sigma(\mathbb{R}) \equiv \mathcal{S}(\mathbb{R})$. See Figure 6 . 


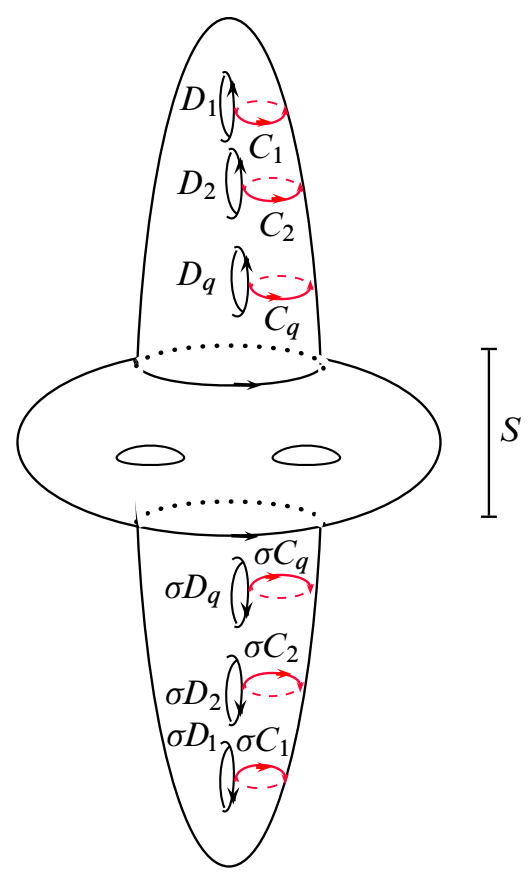

Figure 6: Connected sum 2

There is an equivariant projection $\pi: \Sigma \rightarrow \mathcal{S}$ obtained by collapsing the complement of a collar of $\partial D$ in $\mathcal{C}_{q} \times\{1\}$ to $x_{0}$ and the complement of the corresponding collar of $\sigma(\partial D)$ in $\mathcal{C}_{q} \times\{\sigma\}$ to $\sigma * x_{0}$. Note that $\pi:(\Sigma, \stackrel{\circ}{\mathcal{S}}) \rightarrow(\mathcal{S}, \stackrel{\circ}{\mathcal{S}})$ is a map of pairs inducing a map of long exact sequences:

$$
\begin{array}{r}
\cdots \rightarrow H_{\mathrm{Br}}^{*-1, \bullet}(\stackrel{\circ}{\mathcal{S}} ; \underline{\mathbb{Z}}) \stackrel{\hat{\delta}}{\rightarrow} H_{\mathrm{Br}}^{*, \bullet}(\mathcal{S}, \stackrel{\circ}{\mathcal{S}} ; \underline{\mathbb{Z}}) \stackrel{\hat{b}}{\rightarrow} H_{\mathrm{Br}}^{*, \bullet}(\mathcal{S} ; \underline{\mathbb{Z}}) \stackrel{\hat{r}}{\rightarrow} H_{\mathrm{Br}}^{*, \bullet}(\stackrel{\circ}{\mathcal{S}} ; \underline{\mathbb{Z}}) \rightarrow \cdots \\
\quad \downarrow \\
\downarrow
\end{array}
$$

Remark 4.5 In a similar fashion, one has equivariant projections $\mathcal{S} \rightarrow \mathcal{S} / \stackrel{\mathcal{S}}{\text { and }}$

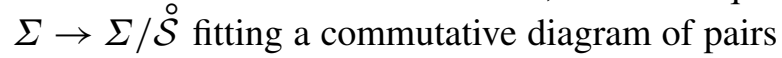

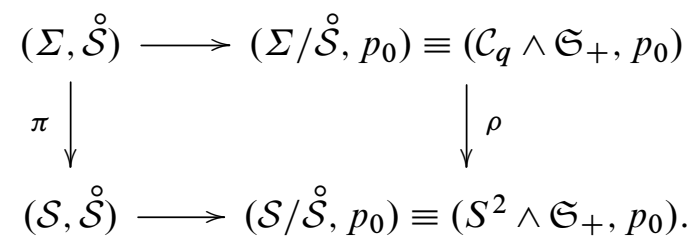


The map $\rho^{*}$ in the exact sequence can be identified with the map in reduced cohomology

$$
\begin{aligned}
\rho^{*}: \widetilde{H}_{\mathrm{Br}}^{*, \bullet}\left(S^{2} \wedge \mathfrak{S}_{+} ; \underline{\mathbb{Z}}\right) \cong & \widetilde{H}_{\text {sing }}^{*}\left(S^{2} ; \mathbb{Z}\right) \otimes \mathbb{Z}\left[\xi, \xi^{-1}\right] \\
& \stackrel{\varrho \otimes 1}{\longrightarrow} \widetilde{H}_{\mathrm{Br}}^{*, \bullet}\left(\mathcal{C}_{q} \wedge \mathfrak{S}_{+} ; \underline{\mathbb{Z}}\right) \cong \widetilde{H}_{\text {sing }}^{*}\left(\mathcal{C}_{q} ; \mathbb{Z}\right) \otimes \mathbb{Z}\left[\xi, \xi^{-1}\right],
\end{aligned}
$$

where $\varrho: \mathcal{C}_{q} \rightarrow S^{2}$ collapses $\stackrel{\circ}{\mathcal{C}}_{q}$ to a point; see Lemma 2.12 . Hence, $\rho^{*}$ is injective and gives an isomorphism in cohomological dimension 2 :

$$
\tilde{H}_{\mathrm{Br}}^{2, \bullet}(\mathcal{S}, \stackrel{\mathcal{S}}{\mathbb{Z}}) \stackrel{\cong}{\rightrightarrows} \tilde{H}_{\mathrm{Br}}^{2, \bullet}(\Sigma, \stackrel{\circ}{\mathcal{S}} ; \underline{\mathbb{Z}}) .
$$

Lemma 4.6 In the exact sequence above, the following hold:

(a) The homomorphism $\rho^{*}$ is a natural split monomorphism.

(b) The homomorphism $\pi^{*}$ is a monomorphism.

(c) The square

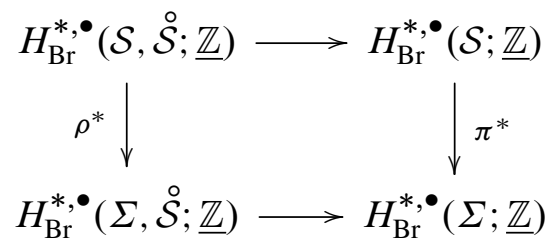

is cocartesian.

(d) This diagram induces a natural isomorphism of $\mathcal{B}$-modules,

$$
H_{\mathrm{Br}}^{*, \bullet}(\Sigma ; \underline{\mathbb{Z}}) \cong H_{\mathrm{Br}}^{*, \bullet}(\mathcal{S} ; \underline{\mathbb{Z}}) \oplus \mathbb{Z}\left[\xi, \xi^{-1}\right] \otimes H_{\text {sing }}^{1}\left(\mathcal{C}_{q} ; \mathbb{Z}\right) .
$$

Proof The first assertion follows from the corresponding fact in singular cohomology and Lemma 2.12, as explained in Remark 4.5. Simple diagram chases then show both (b) and (c).

It follows from assertion (c) that one has an isomorphism

$$
\left(H_{\mathrm{Br}}^{*, \bullet}(\mathcal{S} ; \underline{\mathbb{Z}}) \oplus H_{\mathrm{Br}}^{*, \bullet}(\Sigma, \mathcal{S} ; \underline{\mathbb{Z}})\right) / H_{\mathrm{Br}}^{*, \bullet}(\mathcal{S}, \stackrel{\circ}{\mathcal{S}} ; \underline{\mathbb{Z}}) \stackrel{\cong}{\rightarrow} H_{\mathrm{Br}}^{*, \bullet}(\Sigma ; \underline{\mathbb{Z}})
$$

induced by sending $(u, v) \in H_{\mathrm{Br}}^{*, \bullet}(\mathcal{S} ; \underline{\mathbb{Z}}) \oplus H_{\mathrm{Br}}^{*, \bullet}(\Sigma, \mathcal{S} ; \underline{\mathbb{Z}})$ to $\pi^{*}(u)-b(v) \in H_{\mathrm{Br}}^{*, \bullet}(\Sigma ; \underline{\mathbb{Z}})$. Denote by $[u, v]$ the equivalence class of $(u, v)$ in the quotient above, and use the corresponding isomorphism to identify $[u, v] \equiv \pi^{*}(u)-b(v)$.

Now, denote by $\langle v\rangle$ the class of $v$ in the quotient $H_{\mathrm{Br}}^{*, \bullet}(\Sigma, \stackrel{\circ}{\mathcal{S}} ; \underline{\mathbb{Z}}) / H_{\mathrm{Br}}^{*, \bullet}(\mathcal{S}, \stackrel{\circ}{\mathcal{S}} ; \underline{\mathbb{Z}})$ and let

$$
\mathrm{sp}: H_{\mathrm{Br}}^{*, \bullet}(\Sigma, \stackrel{\circ}{\mathcal{S}} ; \underline{\mathbb{Z}}) \rightarrow H_{\mathrm{Br}}^{*, \bullet}(\mathcal{S}, \stackrel{\circ}{\mathcal{S}} ; \underline{\mathbb{Z}})
$$


be the natural splitting described in (a). It follows easily from the definitions that

$$
\begin{aligned}
& \psi: \frac{\left(H_{\mathrm{Br}}^{*, \bullet}(\mathcal{S} ; \underline{\mathbb{Z}}) \oplus H_{\mathrm{Br}}^{*, \bullet}(\Sigma, \mathcal{\circ} ; \underline{\mathbb{Z}})\right)}{H_{\mathrm{Br}}^{*, \bullet}(\mathcal{S}, \stackrel{\circ}{\mathcal{S}} ; \underline{\mathbb{Z}})} \rightarrow H_{\mathrm{Br}}^{*, \bullet}(\mathcal{S} ; \underline{\mathbb{Z}}) \oplus\left(H_{\mathrm{Br}}^{*, \bullet}(\Sigma, \stackrel{\circ}{\mathcal{S}} ; \underline{\mathbb{Z}}) / H_{\mathrm{Br}}^{*, \bullet}(\mathcal{S}, \stackrel{\circ}{\mathcal{S}} ; \underline{\mathbb{Z}})\right), \\
& {[u, v] } \mapsto(u-\widehat{b}(\operatorname{sp}(v)),\langle v\rangle),
\end{aligned}
$$

is well defined and an isomorphism of $\mathcal{B}$-modules. Finally, we use the natural isomorphism

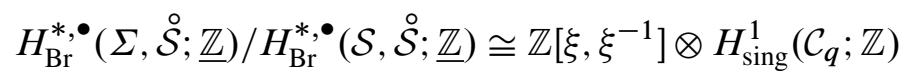

from Lemma 2.12 to complete the proof.

The identification

$$
H_{\mathrm{Br}}^{*, \bullet}(\Sigma ; \underline{\mathbb{Z}}) \cong H_{\mathrm{Br}}^{*, \bullet}(\mathcal{S} ; \underline{\mathbb{Z}}) \oplus \mathbb{Z}\left[\xi, \xi^{-1}\right] \otimes H_{\text {sing }}^{1}\left(\mathcal{C}_{q} ; \mathbb{Z}\right)
$$

is useful to determine the product structure of $H_{\mathrm{Br}}^{*, \bullet}(\Sigma ; \underline{\mathbb{Z}})$. Since $\pi^{*}: H_{\mathrm{Br}}^{*, \bullet}(\mathcal{S} ; \underline{\mathbb{Z}}) \rightarrow$ $H_{\mathrm{Br}}^{*, \bullet}(\Sigma ; \underline{\mathbb{Z}})$ is an injective ring homomorphism, we can see $H_{\mathrm{Br}}^{*, \bullet}(\mathcal{S} ; \underline{\mathbb{Z}})$ as a subring of the cohomology of $\Sigma$ and one needs only to understand products of the form $\gamma \cdot v$ and $v \cdot w$, where $\gamma \in H_{\mathrm{Br}}^{*, \bullet}(\mathcal{S} ; \underline{\mathbb{Z}})$ and $v, w \in H_{\text {sing }}^{1}\left(\mathcal{C}_{q} ; \mathbb{Z}\right) \otimes \mathbb{Z}\left[\xi, \xi^{-1}\right]$.

In what follows let $\eta_{o}$ denote the generator of $H_{\text {sing }}^{2}\left(\mathcal{C}_{q} ; \mathbb{Z}\right)$ and let $\hat{\eta}_{o}$ be the generator of $H_{\text {sing }}^{2}\left(S^{2} ; \mathbb{Z}\right)$ such that $\varrho^{*}\left(\hat{\eta}_{o}\right)=\eta_{o}$. Hence the isomorphism $\rho^{*}$ defined in (33) sends

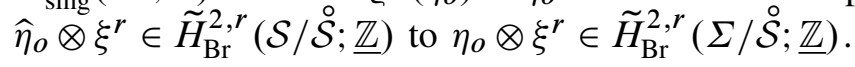

Lemma 4.7 Using the notation above, consider classes $v=\xi^{r} \otimes \mathrm{v}, w=\xi^{s} \otimes \mathrm{w}$ in $\mathbb{Z}\left[\xi, \xi^{-1}\right] \otimes H_{\text {sing }}^{1}\left(\mathcal{C}_{q} ; \mathbb{Z}\right)$, and $\gamma \in H_{\mathrm{Br}}^{n, p}(\mathcal{S} ; \underline{\mathbb{Z}})$, with $n \geq 1$. Then the following hold in $H_{\mathrm{Br}}^{*, \bullet}(\Sigma ; \underline{\mathbb{Z}})$ :

(a) $\gamma \cdot v=0$.

(b) Write $\mathrm{v} \cup \mathrm{w}=n(\mathrm{v}, \mathrm{w}) \eta_{0} \in H_{\text {sing }}^{2}\left(\mathcal{C}_{q} ; \mathbb{Z}\right)$, with $n(\mathrm{v}, \mathrm{w}) \in \mathbb{Z}$; then

$$
v \cdot w=n(\mathrm{v}, \mathrm{w}) \hat{b}\left(\xi^{r+s} \hat{\eta}_{0}\right),
$$

where $\hat{b}$ is indicated in the sequence (32).

Proof We start with a simple description of the elements in $\mathbb{Z}\left[\xi, \xi^{-1}\right] \otimes H_{\text {sing }}^{1}\left(\mathcal{C}_{q} ; \mathbb{Z}\right)$. It is usual to describe the first singular homology group of $\mathcal{C}_{q}$ via a symplectic basis $\left\{C_{1}, \ldots, C_{q} ; D_{1}, \ldots, D_{q}\right\}$, where $C_{i}$ and $D_{i}$ are represented by smooth embedded circles that intersect transversely at a point, as in Figure 6. We may assume that these circles lie in $\mathcal{C}_{q}-\hat{D}$, where $\hat{D}$ is the open disk in the construction of $\Sigma$. Hence, one obtains embeddings $f_{i}: S^{1} \vee S^{1} \rightarrow \mathcal{C}_{q}$ that induce equivariant embeddings 
$F_{i}:\left(S^{1} \vee S^{1}\right) \times \mathfrak{S} \rightarrow \Sigma$. In particular, if $\zeta \in \widetilde{H}_{1,1-r}^{\mathrm{Br}}\left(\left(S^{1} \vee S^{1}\right) \times \mathfrak{S} ; \underline{\mathbb{Z}}\right)$ one obtains a class $\vartheta_{F_{i *}(\zeta)} \in H_{\mathrm{Br}}^{1, r}(\Sigma ; \underline{\mathbb{Z}})$, Poincaré dual to $F_{i *}(\zeta)$. Using the fact that the summand $\mathbb{Z}\left[\xi, \xi^{-1}\right] \otimes H_{\text {sing }}^{1}\left(\mathcal{C}_{q} ; \mathbb{Z}\right)$ is torsion free in $H_{\mathrm{Br}}^{*, \bullet}(\Sigma ; \underline{\mathbb{Z}})$ together with the forgetful functor from equivariant to singular cohomology, one verifies that any element in this summand is a sum of elements of the form $\vartheta_{F_{i *}(\zeta)}$.

Now, given $\gamma \in H_{\mathrm{Br}}^{n, p}(\mathcal{S} ; \underline{\mathbb{Z}})$ the element $F_{i}^{*}(\gamma) \in H_{\mathrm{Br}}^{n, p}\left(\left(S^{1} \vee S^{1}\right) \times \mathfrak{S} ; \underline{\mathbb{Z}}\right)$ is completely determined by the forgetful functor, according to Lemma 2.12. Hence $F_{i}^{*}(\gamma)=0$, whenever $n \geq 2$, as one can clearly see in singular cohomology. It follows that $\left(\gamma \cdot \vartheta_{F_{i *}(\zeta)}\right) \cap[\Sigma]=\gamma \cap F_{i *}(\zeta)=F_{i *}\left(F_{i}^{*}(\gamma) \cap \zeta\right)=0$. This shows the first identity.

Consider $v=\xi^{r} \otimes \mathrm{v}, w=\xi^{s} \otimes \mathrm{w} \in H_{\mathrm{Br}}^{1, \bullet}(\Sigma, \stackrel{\circ}{\mathcal{S}} ; \underline{\mathbb{Z}}) \cong H_{\mathrm{Br}}^{1, \bullet}\left(\Sigma / \mathcal{S}, p_{0} ; \underline{\mathbb{Z}}\right)$.

Using the observation preceding the lemma and the notation in (32) we see that $b(v) \cdot b(w)=b(v \cdot w)=n(\mathrm{v}, \mathrm{w}) b\left(\xi^{r+s} \eta_{0}\right)$. It follows from Remark 4.5 that $\rho^{*}$ is an isomorphism in cohomological dimension 2. Therefore, $\xi^{r+s} \eta_{0}=\rho^{*}\left(\xi^{r+s} \hat{\eta}_{0}\right)$, and hence

$$
b(v) \cdot b(w)=n(v, w) b\left(\rho^{*}\left(\xi^{r+s} \hat{\eta}_{0}\right)\right)=n(v, w) \pi^{*}\left(\hat{b}\left(\xi^{r+s} \hat{\eta}_{0}\right)\right) .
$$

This concludes the proof.

It follows from this lemma that the key ingredient to determine the product structure on the cohomology of $\Sigma$ is the homomorphism $\hat{b}$ appearing in (32). The following cases are of particular interest.

Lemma 4.8 Let $\stackrel{\circ}{\mathcal{S}} \subset \mathcal{S}$ be as above, and consider the sequence

$$
H_{\mathrm{Br}}^{1, \bullet}(\stackrel{\circ}{\mathcal{S}} ; \underline{\mathbb{Z}}) \stackrel{\widehat{\delta}}{\longrightarrow} H_{\mathrm{Br}}^{2, \bullet}(\mathcal{S}, \stackrel{\circ}{\mathcal{S}} ; \underline{\mathbb{Z}}) \equiv \mathbb{Z}\left[\xi, \xi^{-1}\right] \cdot \hat{\eta}_{0} \stackrel{\hat{b}}{\longrightarrow} H_{\mathrm{Br}}^{2, \bullet}(\mathcal{S} ; \underline{\mathbb{Z}}) \stackrel{\hat{r}}{\longrightarrow} H_{\mathrm{Br}}^{2, \bullet}(\stackrel{\circ}{\mathcal{S}} ; \underline{\mathbb{Z}}) .
$$

Then for $\mathcal{S}=B, \mathbb{P}_{\mathbb{R}}^{1}$ or $\mathcal{S}=\Sigma_{1,0}^{0}$, the homomorphism $\hat{b}$ is completely determined by the following data, in the notation of Lemma 4.7.

\begin{tabular}{c|c|c}
$\mathcal{S}$ & $\hat{b}\left(\xi^{-1+2 s} \hat{\eta}_{0}\right)$ & $\hat{b}\left(\xi^{-2+2 s} \hat{\eta}_{0}\right)$ \\
\hline$B$ & $\tau^{-1+s} \eta$ & $\tau^{-2+s} \varepsilon^{2}$ \\
$\mathbb{P}_{\mathbb{R}}^{1}$ & $\tau^{s} \alpha \eta$ & $\tau^{s} \theta \eta$ \\
$\Sigma_{1,0}^{0}$ & $\tau^{-1+s} \eta$ & $\tau^{-2+s} \varepsilon S$
\end{tabular}

Table 7: Values of $\hat{b}$ 
Proof Since $\hat{b}$ is a morphism of $\mathcal{B}$-modules and $\tau$ acts in $\mathbb{Z}\left[\xi, \xi^{-1}\right]$ as multiplication by $\xi^{2}$, it suffices to consider the case of $s \leq 0$.

If $\mathcal{S}=B$, one has

$$
H_{\mathrm{Br}}^{2, \bullet}(\mathcal{S} ; \underline{\mathbb{Z}})=\mathbb{Z}\left[\tau, \tau^{-1}\right] \cdot \eta \oplus \mathbb{F}_{2}\left[\tau, \tau^{-1}\right] \cdot \varepsilon^{2}
$$

and $\stackrel{\circ}{\mathcal{S}}$ is equivariantly homotopy-equivalent to $S_{a}^{1}$ and hence $H_{\mathrm{Br}}^{2, \bullet}(\stackrel{\circ}{\mathcal{S}} ; \underline{\mathbb{Z}})=0$; see [10, page 4711]. It follows that for $\bullet=-1+2 s$ the exact sequence in the statement becomes

$$
H_{\mathrm{Br}}^{1,-1+2 s}\left(S_{a}^{1} ; \underline{\mathbb{Z}}\right) \stackrel{\widehat{\delta}}{\longrightarrow} \mathbb{Z} \cdot\left(\xi^{-1+2 s} \hat{\eta}_{0}\right) \stackrel{\widehat{b}}{\longrightarrow} \mathbb{Z} \cdot\left(\tau^{-1+s} \eta\right) \stackrel{\hat{r}}{\longrightarrow} 0 .
$$

and for $\bullet=-2+2 s$

$$
H_{\mathrm{Br}}^{1,-2+2 s}\left(S_{a}^{1} ; \underline{\mathbb{Z}}\right) \stackrel{\widehat{\delta}}{\longrightarrow} \mathbb{Z} \cdot\left(\xi^{-2+2 s} \hat{\eta}_{0}\right) \stackrel{\widehat{b}}{\longrightarrow} \mathbb{F}_{2} \cdot\left(\tau^{-2+s} \varepsilon^{2}\right) \stackrel{\widehat{r}}{\longrightarrow} 0 .
$$

Since the groups involved are either free or trivial, the result now follows from an application of the forgetful functor to singular cohomology, to determine the correct sign.

When $\mathcal{S}=\mathbb{P}_{\mathbb{R}}^{1}$ one has an equivariant homotopy equivalence $\stackrel{\circ}{\mathcal{S}} \simeq S^{1,0}$. Hence, when $\bullet=-1+2 s$, the exact sequence in the lemma becomes

$$
0 \stackrel{\widehat{\delta}}{\longrightarrow} \mathbb{Z} \cdot\left(\xi^{-1+2 s} \hat{\eta}_{0}\right) \stackrel{\hat{b}}{\longrightarrow} \mathbb{Z} \cdot\left(\tau^{s} \alpha \eta\right) \stackrel{\hat{r}}{\longrightarrow} 0,
$$

and the result follows. For $\bullet=-2+2 s$ one has

$$
\mathbb{Z} \cdot\left(\tau^{s} \alpha \mathrm{Y}\right) \stackrel{\hat{\delta}}{\longrightarrow} \mathbb{Z} \cdot\left(\xi^{-2+2 s} \hat{\eta}_{0}\right) \stackrel{\hat{b}}{\longrightarrow} \mathbb{F}_{2} \cdot\left(\tau^{s} \theta \eta\right) \stackrel{\hat{r}}{\longrightarrow} 0
$$

(see Section 3.2.1). Hence, $\hat{b}$ must send $\xi^{-2+2 s} \hat{\eta}_{0}$ to the generator $\tau^{s} \theta \eta$.

Finally, when $\mathcal{S}=\Sigma_{1,0}^{0}$ the subspace $\mathcal{\mathcal { S }}$ is equivariantly homotopy equivalent to the pushout of two equivariant inclusions $f: \mathfrak{S} \rightarrow S_{a}^{1}$ and $g: \mathfrak{S} \rightarrow S^{1,0} \times \mathfrak{S}$. In particular, $H_{\mathrm{Br}}^{2, \bullet}(\stackrel{\circ}{\mathcal{S}} ; \underline{\mathbb{Z}})=0$. Hence, when $\bullet=-1+2 s$, the exact sequence in the lemma becomes

$$
H_{\mathrm{Br}}^{1,-1+2 s}(\stackrel{\circ}{\mathcal{S}} ; \underline{\mathbb{Z}}) \stackrel{\widehat{\delta}}{\longrightarrow} \mathbb{Z} \cdot\left(\xi^{-1+2 s} \hat{\eta}_{0}\right) \stackrel{\hat{b}}{\longrightarrow} \mathbb{Z} \cdot\left(\tau^{-1+s} \eta\right) \stackrel{\hat{r}}{\longrightarrow} 0,
$$

which together with an application of $\varphi$ gives the result. For $\bullet=-2+2 s$ one has

$$
H_{\mathrm{Br}}^{1,-2+2 s}(\stackrel{\circ}{\mathcal{S}} ; \underline{\mathbb{Z}}) \stackrel{\hat{\delta}}{\longrightarrow} \mathbb{Z} \cdot\left(\xi^{-2+2 s} \widehat{\eta}_{0}\right) \stackrel{\hat{b}}{\longrightarrow} \mathbb{F}_{2} \cdot\left(\tau^{-2+s} \varepsilon S\right) \stackrel{\hat{r}}{\longrightarrow} 0 .
$$

Hence, $\hat{b}$ must send $\xi^{-2+2 s} \widehat{\eta}_{0}$ to the generator $\tau^{-2+s} \varepsilon \mathrm{S}$. 


\subsection{Curves without real points}

Here we prove statements (III) and (IV) in Theorem 4.1. Using the gluing construction of Section 4.1.2 with $\mathcal{S}=B$ and $\mathcal{S}=\Sigma_{1,0}^{0}$, we obtain all possible types of curves without real points: $\Sigma_{g, 0}^{0}$. We will now compute their Bredon cohomology rings using Lemmas 4.7 and 4.8 and the computations of the basic cases described in Section 3.

The same construction with $\mathcal{S}=\mathbb{P}_{\mathbb{R}}^{1}$ yields curves of type $\Sigma_{2 q, 1}^{1}$, which will also be needed hence we will also consider this case below.

Proposition 4.9 Applying the construction of Section 4.1.2 with $\mathcal{S}=B, \mathbb{P}_{\mathbb{R}}^{1}$, and $\Sigma_{1,0}^{0}$, we obtain a real curve $\Sigma$ of type $\Sigma_{2 q, 0}^{0}, \Sigma_{2 q, 1}^{1}$ and $\Sigma_{2 q+1,0}^{0}$, respectively.

The $\mathcal{B}$-module structure of $H_{\mathrm{Br}}^{*, \bullet}(\Sigma ; \underline{\mathbb{Z}})$ is given by

$$
H_{\mathrm{Br}}^{*, \bullet}(\Sigma(\mathbb{C}) ; \underline{\mathbb{Z}}) \cong H_{\mathrm{Br}}^{*, \bullet}(\mathcal{S} ; \underline{\mathbb{Z}}) \oplus \mathbb{Z}\left[\xi, \xi^{-1}\right](\boldsymbol{u}),
$$

where $\boldsymbol{u}=\left(u_{1}, \ldots, u_{2 q}\right)$ and $\operatorname{deg} u_{i}=(1,0)$. The ring structure of $H_{\mathrm{Br}}^{*, \bullet}(\Sigma(\mathbb{C}) ; \underline{\mathbb{Z}})$ is determined by the fact that $H_{\mathrm{Br}}^{*, \bullet}(\mathcal{S} ; \underline{\mathbb{Z}})$ is a subring and the following set of relations:

(i) $x \cdot y=0$ for $x \in H_{\mathrm{Br}}^{>0, \bullet}(\mathcal{S} ; \underline{\mathbb{Z}})$ and $y \in \mathbb{Z}\left[\xi, \xi^{-1}\right](\boldsymbol{u})$.

(ii) $\xi^{k} u_{i} \cdot \xi^{l} u_{j}=(-1)^{k} \delta_{i+j, 2 q+1} \Gamma_{k+l}^{\Sigma}$, where $\Gamma_{s}^{\Sigma}$ is displayed in Table 5 above.

Furthermore, in Table 6 the images of the generators $u_{1}, \ldots, u_{2 q}$ under the functor $\varphi$ are expressed in terms $\left\{\vartheta_{\boldsymbol{\alpha}}, \vartheta_{\boldsymbol{\beta}}\right\}$.

Proof By Lemma 4.6, we have $H_{\mathrm{Br}}^{*, \bullet}(\Sigma ; \underline{\mathbb{Z}}) \cong H_{\mathrm{Br}}^{*, \bullet}(\mathcal{S} ; \underline{\mathbb{Z}}) \oplus \mathbb{Z}\left[\xi, \xi^{-1}\right] \otimes H_{\text {sing }}^{1}\left(\mathcal{C}_{q} ; \mathbb{Z}\right)$. Let $\{\boldsymbol{C}, \boldsymbol{D}\}$ be a homology basis as displayed in Figure 6. Starting with the cohomology basis $\left\{\vartheta_{\boldsymbol{C}}, \vartheta_{\boldsymbol{D}}\right\}$ and tracing through the isomorphisms of Lemmas 4.6 and 2.12 we arrive at a set of generators $\xi^{k} u_{i}, \xi^{k} u_{i+q} \in H_{\mathrm{Br}}^{1, k}(\Sigma ; \underline{Z})$ corresponding respectively to $\xi^{k} \vartheta_{C_{i}}$ and $\xi^{k} \vartheta_{D_{q+1-i}}$ such that (34) holds. Furthermore, computing the image under $\varphi$ of $\xi^{k} u_{i}$, in terms of the symplectic basis $\left\{\vartheta_{\boldsymbol{\alpha}}, \vartheta_{\boldsymbol{\beta}}\right\}$ (with $\{\boldsymbol{\alpha}, \boldsymbol{\beta}\}$ as in Table 3 ), we obtain the expressions given in Table 6.

This proves Theorem 4.1(III), (IV).

\subsection{Curves with real points}

Here we prove statements (I) and (II) in Theorem 4.1.

4.3.1 Separating curves Using the equivariant connected sum which was described in Section 4.1.1 one constructs every curve of separating type $\Sigma_{g, r}^{1}$ from curves of 
type $\Sigma_{1,2}^{1}, \Sigma_{2 q, 1}^{1}$ and $\Sigma_{0,1}^{1}$, by observing that

$$
\Sigma_{g, r}^{1}=\Sigma_{r-1, r}^{1} \#_{\mathrm{D}^{2,1}} \Sigma_{g-r+1,1}^{1}=\Sigma_{r-1, r}^{1} \#_{\mathrm{D}^{2,1}} \Sigma_{2 q, 1}^{1} .
$$

From Proposition 4.9 one obtains a description

$$
H_{\mathrm{Br}}^{*, \bullet}\left(\Sigma_{2 q, 1}^{1}(\mathbb{C}) ; \underline{Z}\right) \cong \mathcal{B} \cdot \mathbf{1} \oplus \mathbb{Z}\left[\xi, \xi^{-1}\right](\boldsymbol{u}) \oplus \mathcal{B} \cdot \eta,
$$

as a $\mathcal{B}$-module, where $\boldsymbol{u}:=\left(u_{1}, \ldots, u_{2 q}\right)$ and $\operatorname{deg} u_{i}=(1,0)$, so that the ring structure is determined by the relation ( of Theorem 4.1. Finally, Lemma 4.3 applied to the equivariant connected sum construction in (35) together with Example 4.4 concludes the proof of Theorem 4.1(I).

4.3.2 Nonseparating curves The curves of nonseparating type can be constructed as

$$
\Sigma_{g, r}^{0}=\Sigma_{g+1-r, 1}^{0} \#_{\mathrm{D}^{2,1}} \Sigma_{r-1, r}^{1}=\underbrace{\left(\Sigma_{1,1}^{0} \#_{\mathrm{D}^{2,1}} \cdots \#_{\mathrm{D}^{2,1}} \Sigma_{1,1}^{0}\right)}_{g+1-r} \#_{\mathrm{D}^{2,1}} \Sigma_{r-1, r}^{1} .
$$

Now, using Lemma 4.3 inductively, together with Example 4.4 and Proposition 3.6 one concludes the proof of Theorem 4.1(II).

\section{The Deligne cohomology of real curves}

In this section we compute the Deligne cohomology ring of a real curve $\Sigma$, organizing the calculation into the same four cases of Theorem 4.1. We start with some preliminaries including general technical results and the cases of the curves $B=\Sigma_{0,0}^{0}$ and $\Sigma_{1,0}^{0}$, as these will be used in the statement of the general result.

\subsection{Preliminaries}

5.1.1 Brauer-Severi curve Using the long exact sequence (12) and the description of the Bredon bigraded cohomology of the Brauer-Severi curve in Proposition 3.1 we obtain the following result.

Proposition 5.1 Let $B$ be the Brauer-Severi curve. One has an isomorphism of $\mathcal{D}_{\mathrm{EV}}-$ modules

$$
H_{\mathcal{D} / \mathbb{R}}^{*}(B ; \mathbb{Z}(\bullet)) \cong\left(\mathcal{D}_{\mathrm{EV}} / J_{\mathrm{EV}}^{3}\right) \oplus\left(\mathcal{D}_{\mathrm{EV}} / J_{\mathrm{EV}}\right) \cdot \eta,
$$

where $J_{\mathrm{EV}}$ is the ideal introduced in Remark 2.11. There is a ring isomorphism

$$
H_{\mathcal{D} / \mathbb{R}}^{*}(B ; \mathbb{Z}(\bullet)) \cong \frac{\mathcal{D}_{\mathrm{EV}}[\eta]}{J_{\mathrm{EV}}\left(J_{\mathrm{EV}}^{2}+\langle\eta\rangle\right)},
$$

where $\eta$ has degree $(2,1)$. 
5.1.2 Genus 1 curves without real points $\boldsymbol{\Sigma}_{\mathbf{1 , 0}}^{\mathbf{0}}$ In a similar fashion, using long exact sequences, the Bredon cohomology ring structure and dimensional considerations the next result follows in a straightforward manner.

Proposition 5.2 For $\Sigma=\Sigma_{1,0}^{0}$ one has an isomorphism of $\mathcal{D}_{\mathrm{EV}}$-modules

$$
H_{\mathcal{D} / \mathbb{R}}^{*}\left(\Sigma_{1,0}^{0} ; \mathbb{Z}(\bullet)\right) \cong \mathcal{D}_{\mathrm{EV}} / J_{\mathrm{EV}}^{2} \cdot \mathbf{1} \oplus \widetilde{\mathcal{D}}_{\mathrm{EV}} / J_{\mathrm{EV}}^{2}\{\mathrm{~s}\} \oplus \mathrm{X}_{\mathcal{D}_{\mathrm{EV}}}^{\Sigma}(\mathrm{T}) \oplus \mathcal{D}_{\mathrm{EV}} / J_{\mathrm{EV}} \cdot \eta,
$$

where $\mathrm{S}, \mathrm{T}, \eta$ have degrees $(1,1),(1,0)$ and $(2,1)$, respectively and

$$
\mathrm{X}_{\mathcal{D}_{\mathrm{EV}}}^{\Sigma}(\mathrm{T})=\mathcal{D}_{\mathrm{EV}}\{\mathrm{T}\} /\left(J_{\mathrm{EV}}+\mathscr{F}_{\Sigma}\right) \text {, }
$$

where $\mathscr{S}_{\Sigma}=\mathbb{Z}\left\{\frac{\pi}{t} \mathrm{E} \cdot \mathrm{T}\right\}$, where $t=\operatorname{Im} \int_{\beta_{1}} \omega_{1}$ and $\omega_{1}$ is a holomorphic form adapted to the symplectic basis $\left\{\alpha_{1}, \beta_{1}\right\}$ described in Section 2.1.3. The ring structure is completely determined by:

(1) $(\lambda \cdot s)\left(\lambda^{\prime} \cdot s\right)=\left(\lambda \lambda^{\prime} \varepsilon\right) \cdot s$ for $\lambda, \lambda^{\prime} \in \widetilde{\mathcal{D}}_{\mathrm{EV}}$.

(2) $(\lambda \cdot \mathrm{s}) \mathrm{T}=\lambda \cdot \eta$ for $\lambda \in \widetilde{\mathcal{D}}_{\mathrm{EV}}$.

(3) $(\lambda \cdot \mathrm{s}) \eta=0, \mathrm{~T}^{2}=0$ and $\mathrm{T} \eta=0$.

Remark 5.3 The proposition above can be rephrased in the following way. One has a ring isomorphism

$$
H_{\mathcal{D} / \mathbb{R}}^{*}\left(\Sigma_{1,0}^{0} ; \mathbb{Z}(\bullet)\right) \cong \frac{\mathcal{D}_{\mathrm{EV}}[\mathrm{T}, \eta]+\mathrm{s} \tilde{\mathcal{D}}_{\mathrm{EV}}[\mathrm{s}, \mathrm{T}, \eta]}{I},
$$

where $I=I_{1}+I_{2}+I_{3}+\mathcal{I}_{\Sigma}$ is the sum of the following ideals:

(1) $I_{1}=\left(J_{\mathrm{EV}}+\langle\mathrm{T}\rangle+\langle\eta\rangle\right)^{2}$.

(2) $I_{2}=\left\langle(\lambda \cdot \mathrm{s}) \eta,(\lambda \cdot \mathrm{s}) \mathrm{T}-\lambda \cdot \eta \mid \lambda \in \widetilde{\mathcal{D}}_{\mathrm{EV}}\right\rangle$.

(3) $I_{3}=\left\langle(\lambda \mathrm{s})\left(\lambda^{\prime} \mathrm{s}\right)-\lambda \lambda^{\prime} \varepsilon \mathrm{S} \mid \lambda, \lambda^{\prime} \in \widetilde{\mathcal{D}}_{\mathrm{EV}}\right\rangle$.

(4) $\mathscr{F}_{\Sigma}=\left\langle\frac{\pi}{t} \mathrm{E} \cdot \mathrm{T}\right\rangle$, where $t=\operatorname{im} \int_{\beta_{1}} \omega_{1}$ and $\omega_{1}$ is a holomorphic form adapted to the symplectic basis $\left\{\alpha_{1}, \beta_{1}\right\}$ described in Section 2.1.3.

5.1.3 Technical results The first result has a well-known counterpart for complex varieties and follows basically for the same reason, namely the fact that the Hodge filtration $F^{p} \mathcal{E}_{X}^{*}$ vanishes whenever $\operatorname{dim} X<p$.

Lemma 5.4 Let $X$ be a smooth complete real variety of dimension $d$. Then for $p>d$, there are natural isomorphisms

$b_{\mathbb{R}}: H_{\mathrm{Br}}^{n-1, p}(X, \underline{\mathbb{C} / \mathbb{Z}}) \rightarrow H_{\mathcal{D} / \mathbb{R}}^{n}(X ; \mathbb{Z}(p)), \quad b_{\mathbb{C}}: H_{\text {sing }}^{n-1}(X ; \mathbb{C} / \mathbb{Z}(p)) \rightarrow H_{\mathcal{D} / \mathbb{C}}^{n}(X ; \mathbb{Z}(p))$, 
which are compatible with forgetful functors, ie the following diagram commutes:

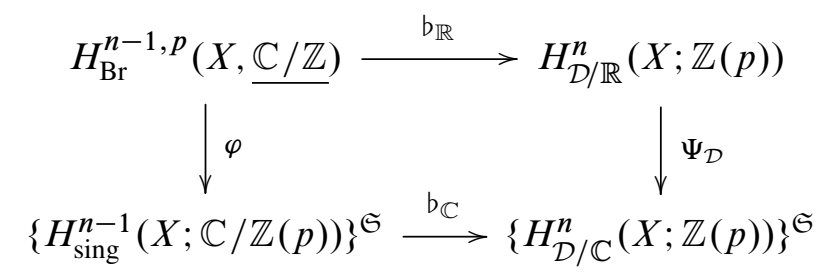

Furthermore, the composition

$$
H_{\mathrm{Br}}^{n-1, p}\left(X(\mathbb{C}) ; \underline{\mathbb{Z}} \stackrel{\mathrm{b}_{\mathbb{R}}}{\longrightarrow} H_{\mathcal{D} / \mathbb{R}}^{n}(X ; \mathbb{Z}(p)) \stackrel{\Psi_{\mathcal{B}}}{\longrightarrow} H_{\mathrm{Br}}^{n, p}(X(\mathbb{C}) ; \underline{\mathbb{Z}})\right.
$$

coincides with the connecting homomorphism induced by the exact sequence

$$
0 \rightarrow \underline{\mathbb{Z}} \rightarrow \underline{\mathbb{C}} \rightarrow \underline{\mathbb{C} / \mathbb{Z}} \rightarrow 0 .
$$

Remark 5.5 Applying the previous result to the case of a point one can identify $\mathcal{D}^{n, p}=H_{\mathrm{Br}}^{n, p}(\mathrm{pt}, \mathbb{C} / \mathbb{Z})$ for all $p>0$. In particular, we will abuse notation and will not distinguish between $\lambda$ and $b_{\mathbb{R}}(\lambda)$ when $\lambda \in H_{\mathrm{Br}}^{*, \geq 1}(\mathrm{pt}, \underline{\mathbb{C} / \mathbb{Z})}$.

The next result comes from natural pairings in the level of coefficients and its proof is left to the reader.

Lemma 5.6 Let $X$ be a $\mathfrak{S}$-space. Then the pairing of $\mathfrak{S}$-modules $\mathbb{C} / \mathbb{Z} \otimes \mathbb{Z} \rightarrow \mathbb{C} / \mathbb{Z}$, given by $[z] \otimes n \mapsto[n z]$ induces pairings

$$
\mathrm{m}_{\mathbb{R}}: H_{\mathrm{Br}}^{n, p}(X, \underline{\mathbb{C} / \mathbb{Z}}) \otimes H_{\mathrm{Br}}^{n^{\prime}, p^{\prime}}(X ; \underline{\mathbb{Z}}) \rightarrow H_{\mathrm{Br}}^{n+n^{\prime}, p+p^{\prime}}(X, \underline{\mathbb{C} / \mathbb{Z}}),
$$

$\mathrm{m}_{\mathbb{C}}: H_{\text {sing }}^{n}(X ; \mathbb{C} / \mathbb{Z}(p)) \otimes H_{\text {sing }}^{n^{\prime}}\left(X ; \mathbb{Z}\left(p^{\prime}\right)\right) \rightarrow H_{\text {sing }}^{n+n^{\prime}}\left(X ; \mathbb{C} / \mathbb{Z}\left(p+p^{\prime}\right)\right)$,

which are compatible with forgetful functors and connecting homomorphisms, ie the following diagram commutes:

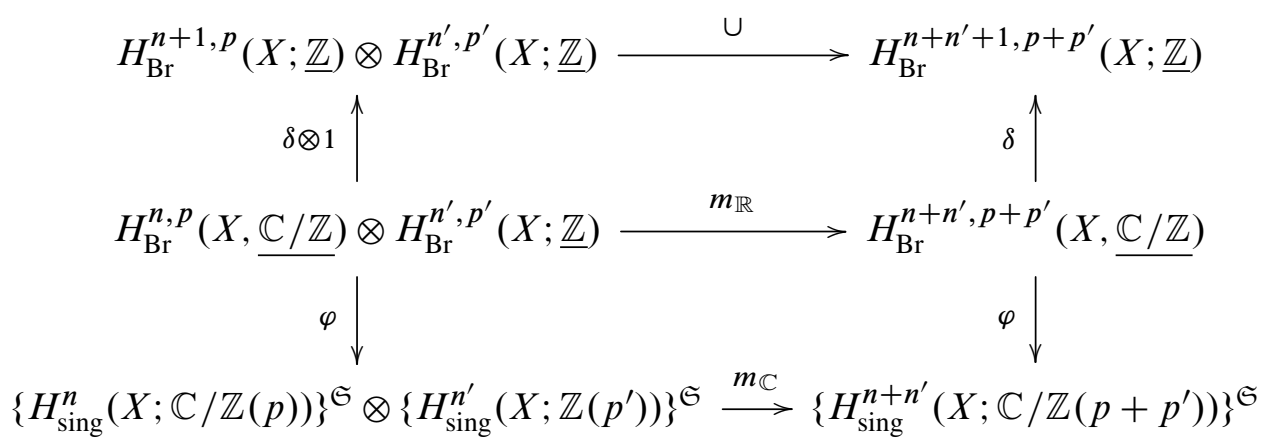


Remark 5.7 It follows from the two propositions above that the cohomology of Spec $\mathbb{C}$ admits an operation

$$
\xi^{-1}: \mathcal{D}_{\mathbb{C}}^{*, p} \longrightarrow \mathcal{D}_{\mathbb{C}}^{*, p-1}
$$

which is 0 when $p=1$ and an isomorphism when $p \neq 1$. For $p \geq 2$ this is obtained by identifying $\xi^{-1}=(2 \pi \boldsymbol{i})^{-1} \in H_{\text {sing }}^{0}(\mathrm{pt} ; \mathbb{Z}(-1))$ and using the multiplication

$$
m_{\mathbb{C}}: H_{\text {sing }}^{*}(\mathrm{pt} ; \mathbb{C} / \mathbb{Z}(p)) \otimes H_{\text {sing }}^{0}(\mathrm{pt} ; \mathbb{Z}(-1)) \longrightarrow H_{\text {sing }}^{*}(\mathrm{pt} ; \mathbb{C} / \mathbb{Z}(p-1)),
$$

described in Lemma 5.6, together with the isomorphism $b_{\mathbb{C}}$ from Lemma 5.4. For $p \leq 0$ one has $\xi^{-1}: H_{\mathcal{D} / \mathbb{C}}^{*}(\mathrm{pt} ; \mathbb{Z}(p)) \stackrel{\cong}{\rightarrow} H_{\text {sing }}^{*}(\mathrm{pt} ; \mathbb{Z}(p))$, in which case we use the isomorphism

$$
\mathbb{Z}(p) \stackrel{\cong}{\rightarrow} \mathbb{Z}(p-1)
$$

given by multiplication by $(2 \pi \boldsymbol{i})^{-1}$ directly on the level of coefficients.

Definition 5.8 For a real curve $\Sigma$, let

$$
\begin{aligned}
& \Psi_{\mathcal{D}}: H_{\mathcal{D} / \mathbb{R}}^{n}(\Sigma ; \mathbb{Z}(p)) \rightarrow H_{\mathcal{D} / \mathbb{C}}^{n}(\Sigma ; \mathbb{Z}(p))^{\mathfrak{S}}, \\
& \Psi_{\mathcal{B}}: H_{\mathcal{D} / \mathbb{R}}^{n}(\Sigma ; \mathbb{Z}(p)) \rightarrow H_{\mathrm{Br}}^{n, p}(\Sigma ; \underline{\mathbb{Z}})
\end{aligned}
$$

be the forgetful functors and denote their product by $\Psi:=\Psi_{\mathcal{D}} \times \Psi_{\mathcal{B}}$.

Lemma 5.9 Let $\Sigma$ be a real curve such that $\Sigma(\mathbb{R}) \neq \varnothing$. Then for all $p>1$ and $n \in \mathbb{Z}, \Psi: H_{\mathcal{D} / \mathbb{R}}^{n}(\Sigma ; \mathbb{Z}(p)) \rightarrow H_{\mathcal{D} / \mathbb{C}}^{n}(\Sigma ; \mathbb{Z}(p))^{\mathfrak{S}} \times H_{\mathrm{Br}}^{n, p}(\Sigma ; \underline{\mathbb{Z}})$ is injective.

Proof For simplicity, we will use $H_{\mathcal{D} / \mathbb{R}}^{n, p}, H_{\mathrm{Br}}^{n, p}, H_{\mathbb{C}}^{n}$ and $H_{\mathbb{Z}(p)}^{n}$ to abbreviate

$$
H_{\mathcal{D} / \mathbb{R}}^{n}(\Sigma ; \mathbb{Z}(p)), \quad H_{\mathrm{Br}}^{n, p}(\Sigma ; \underline{\mathbb{Z}}), \quad H_{\text {sing }}^{n}(\Sigma ; \mathbb{C}) \quad \text { and } \quad H_{\text {sing }}^{n}(\Sigma ; \mathbb{Z}(p)),
$$

respectively. Consider the map of long exact sequences which correspond to the forgetful functor $H_{\mathcal{D} / \mathbb{R}}^{n, p} \rightarrow H_{\mathcal{D} / \mathbb{C}}^{n, p}:$

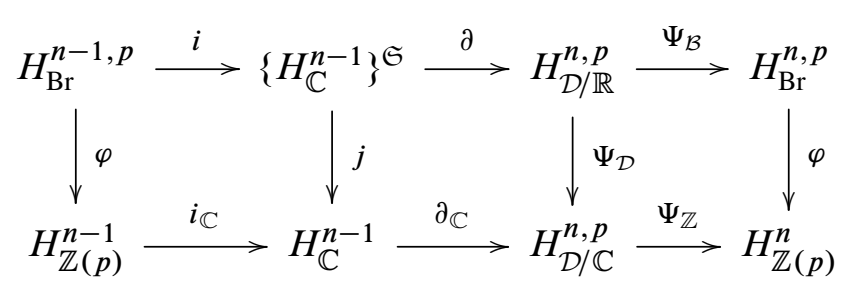

If $c \in H_{\mathcal{D} / \mathbb{R}}^{n, p} \in \operatorname{ker} \Psi$ then $\Psi_{\mathcal{B}}(c)=0$ and there exists $\alpha \in \partial^{-1}(c)$. We have $\partial_{\mathbb{C}} j(\alpha)=$ $\Psi_{\mathcal{D}}(c)=0$, hence $j(\alpha) \in \operatorname{im} i_{\mathbb{C}}$. 
Now, under the hypothesis $\Sigma(\mathbb{R}) \neq \varnothing$ the map

$$
\Psi_{\mathcal{B}}: H_{\mathrm{Br}}^{n-1, p} \rightarrow\left\{H_{\mathbb{Z}(p)}^{n-1}\right\}^{\mathfrak{S}} \subset\left\{H_{\mathbb{C}}^{n-1}\right\}^{\mathfrak{S}}
$$

is onto (see Theorem 4.1). Hence $\alpha \in \operatorname{im} i$ and $c=0$.

Let $\Sigma$ denote a complete smooth complex curve, and let $Z=\left(z_{k j}\right)$ denote the period matrix relative to a symplectic basis $\left\{\underline{\alpha}_{j}, \underline{\beta}_{j} \mid j=1, \ldots, g\right\}$ for $H_{1}(\Sigma, \mathbb{Z})$. Define

$$
\widehat{\omega}_{j}=\sum_{k=1}^{g}\left(\left[z_{k j}\right]_{1} \otimes \vartheta_{\alpha_{k}}-[1]_{1} \otimes \vartheta_{\beta_{k}}\right) \in \mathcal{D}_{\mathbb{C}}^{1,1} \otimes H_{\text {sing }}^{1}(\Sigma, \mathbb{Z}),
$$

where $[z]_{p}$ denotes the class of $z \in \mathbb{C}$ in the quotient $\mathbb{C} / \mathbb{Z}(p)$, for all $p \in \mathbb{Z}$.

Proposition 5.10 Let $\Sigma$ be a complete smooth complex curve. Let $\eta_{o} \in H_{\text {sing }}^{2}(\Sigma ; \mathbb{Z})$ denote the fundamental class of $\Sigma$ determined by a choice of $i=\sqrt{-1}$ and let $\Lambda_{\mathcal{D}_{\mathbb{C}}}\left(\eta_{\mathbb{C}}\right)$ be the (bigraded) exterior algebra on an indeterminate $\eta_{\mathbb{C}}$ of bidegree $(2,1)$ over the ring $\mathcal{D}_{\mathbb{C}}$. Then there is a ring isomorphism

$$
H_{\mathcal{D} / \mathbb{C}}^{*}(\Sigma ; \mathbb{Z}(\bullet)) \cong \Lambda_{\mathcal{D}_{\mathbb{C}}}\left(\eta_{\mathbb{C}}\right) \otimes H_{\text {sing }}^{*}(\Sigma ; \mathbb{Z}) /\left\langle\xi^{-1}(\lambda) \eta_{\mathbb{C}} \otimes 1-\lambda \otimes \eta_{o} \mid \lambda \in \mathcal{D}_{\mathbb{C}}\right\rangle+J_{\Sigma},
$$

where $J_{\Sigma}$ is the "Jacobian ideal"

$$
J_{\Sigma}=\left\langle\widehat{\omega}_{j} \mid j=1, \ldots, g\right\rangle,
$$

with $\widehat{\omega}_{j}$ as in (38) and $\xi^{-1}$ as in (37). Moreover, the action of $\mathfrak{S}$ on $H_{\mathcal{D} / \mathbb{C}}^{\star, \bullet}$ is induced by its joint action on $\mathcal{D}_{\mathbb{C}}$ and $H_{\text {sing }}^{*}(\Sigma ; \mathbb{Z})$ and by letting $\mathfrak{S}$ act freely on $\Lambda_{\mathcal{D}_{\mathbb{C}}}$.

5.1.4 The $\mathcal{D}$-module $\mathbf{X}_{\mathcal{D}}(\boldsymbol{b}, \boldsymbol{u}) \quad$ Let $\boldsymbol{b}=\left(b_{1}, \ldots, b_{s}\right)$ and $\boldsymbol{u}=\left(u_{1}, \ldots, u_{k}\right)$ be sets of variables with $\operatorname{deg} b_{i}=\operatorname{deg} u_{i}=(1,0)$, and let $\mathrm{X}=\left(x_{i j}\right)$ be a $g \times g$ matrix, with $g=k+s$. Write $\mathcal{D}=\mathbb{Z} \oplus \mathbb{Z}\left[\tau^{-1}\right] \cdot \alpha \oplus J \oplus M$ and recall that both $M$ and $J$ are ideals in $\mathcal{D}$ with $M=\mathbb{R}[\tau] \cdot \mathrm{E} \oplus(\tau \mathbb{Z}[\tau] \cdot \mathrm{Y}) \otimes \mathbb{R} / \mathbb{Z}$.

Let $\mathscr{F}_{\mathrm{X}} \subset \mathcal{D} \otimes \mathbb{Z}\{\boldsymbol{b}\} \oplus M \otimes \mathbb{Z}\{\boldsymbol{u}\}$ denote the free abelian subgroup generated by the entries of the $1 \times g$ matrix $(\mathrm{E} \otimes \boldsymbol{u} \mathrm{E} \otimes \boldsymbol{b}) \mathrm{X}$. In other words, $\mathscr{E}_{\mathrm{X}}=\mathbb{Z}\{\boldsymbol{v}\}$ where $\boldsymbol{v}=\left(v_{1}, \ldots, v_{g}\right)$ with

$$
v_{j}=\sum_{i=1}^{k} x_{i j} \mathrm{E} \otimes u_{i}+\sum_{l=1}^{s} x_{k+l, j} \mathrm{E} \otimes b_{l} .
$$

Since $\widetilde{\mathcal{D}} \cdot \mathrm{E}=0$ one concludes $\mathscr{F}_{\mathrm{X}}$ is in fact a $\mathcal{D}$-submodule of $\mathcal{D} \otimes \mathbb{Z}\{\boldsymbol{b}\} \oplus M \otimes \mathbb{Z}\{\boldsymbol{u}\}$. We now introduce a $\mathcal{D}$-module structure on

$$
\mathrm{X}_{\mathcal{D}}(\boldsymbol{u}, \boldsymbol{b})=(\mathcal{D}(\boldsymbol{b}) \oplus M \otimes \mathbb{Z}(\boldsymbol{u})) / \mathscr{F}_{\mathrm{X}} \oplus M \otimes \mathbb{Z}(\xi \boldsymbol{u}) \oplus \mathbb{Z}\left[\xi^{-1}\right](\boldsymbol{u}) .
$$


It follows from the previous paragraph that it suffices to describe how $\mathcal{D}$ multiplies elements in $\mathbb{Z}\left[\xi^{-1}\right](\boldsymbol{u})$. This goes as follows: for each $j=1, \ldots, s$ one has the following relations $\bmod \mathscr{F}_{\mathrm{X}}$ :

(i) $\lambda \cdot u_{j}=\lambda \otimes u_{j}, \lambda \in M$.

(ii) $\lambda \cdot\left(\xi^{-l-1} u_{j}\right)=0$ for all $\lambda \in J \oplus M$ and $l \geq 0$.

(iii) $\varepsilon u_{j}=\frac{1}{2} v_{j}$ (see (39)).

(iv) $\varepsilon \tau^{k} \cdot u_{i}=\left(\tau^{k} \mathrm{Y} \otimes\left[\frac{1}{2}\right]\right) \otimes \xi u_{i}, k \geq 1$.

(v) $J^{2} \cdot u_{i}=\{0\}$.

(vi) $\tau^{-k} \alpha \cdot\left(\xi^{-l} u_{i}\right)=2 \xi^{-2 k-l-2} u_{i}, k, l \geq 0$.

Denote by $\mathrm{X}_{\mathcal{D}}(\boldsymbol{u}), \mathrm{X}_{\mathcal{D}}(\boldsymbol{b}) \subset \mathrm{X}_{\mathcal{D}}(\boldsymbol{u}, \boldsymbol{b})$ the submodules

$$
\begin{gathered}
\mathrm{X}_{\mathcal{D}}(\boldsymbol{u})=M \otimes \mathbb{Z}(\boldsymbol{u}) /\left(\mathscr{F}_{\mathrm{X}} \cap M \otimes \mathbb{Z}(\boldsymbol{u})\right) \oplus M \otimes \mathbb{Z}(\xi \boldsymbol{u}) \oplus \mathbb{Z}\left[\xi^{-1}\right](\boldsymbol{u}), \\
\mathrm{X}_{\mathcal{D}}(\boldsymbol{b})=\operatorname{Image}\left\{\mathcal{D}(\boldsymbol{b}) \longrightarrow \mathrm{X}_{\mathcal{D}}(\boldsymbol{u}, \boldsymbol{b})\right\} .
\end{gathered}
$$

Remark 5.11 Similarly, define $X_{\mathcal{D}_{\mathrm{EV}}}^{\Sigma}(\boldsymbol{u}, \mathrm{T})$ by

(43) $\mathrm{X}_{\mathcal{D}_{\mathrm{EV}}}(\boldsymbol{u}, \mathrm{T})=\left(\mathcal{D}_{\mathrm{EV}}(\mathrm{T}) \oplus M \otimes \mathbb{Z}(\boldsymbol{u})\right) /\left(J_{\mathrm{EV}} \oplus \mathscr{F}_{\mathrm{X}}\right) \oplus M \otimes \mathbb{Z}(\xi \boldsymbol{u}) \oplus \mathbb{Z}\left[\xi^{-1}\right](\boldsymbol{u})$;

see Remark 2.11. Also, define $\mathrm{X}_{\mathcal{D}_{\mathrm{EV}}}(\boldsymbol{u})$ in the same way $\mathrm{X}_{\mathcal{D}}(\boldsymbol{u})$ is defined in (41).

\subsection{Cohomology ring}

Given a real curve $\Sigma$ of genus $g$, we define a $g \times g$ matrix $\mathrm{X}^{\Sigma}=\Lambda \mathrm{T}^{-1}$, where $\mathrm{T}$ is the imaginary part of the period matrix, as in Proposition 2.4, and $\Lambda$ is defined in Table 8.

\begin{tabular}{ccccc}
$\Sigma$ & $\Sigma_{g, r}^{1}$ & $\Sigma_{g, r}^{0}$ & $\Sigma_{2 q, 0}^{0}$ & $\Sigma_{2 q+1,0}^{0}$ \\
\hline$\Lambda$ & $\left(\begin{array}{cc}J_{2 q} & 0 \\
0 & -2 I_{r-1}\end{array}\right)$ & $\left(\begin{array}{cc}I_{2 q} & 0 \\
0 & -2 I_{r-1}\end{array}\right)$ & $J_{2 q}$ & $\left(\begin{array}{cc}J_{2 q} & 0 \\
0 & -1\end{array}\right)$
\end{tabular}

Table 8

The goal of this section is to establish the following result.

Theorem 5.12 Let $\Sigma$ be a real curve, and consider sets of variables

$$
\boldsymbol{a}:=\left\{a_{1}, \ldots, a_{r-1}\right\}, \quad \boldsymbol{b}=\left\{b_{1}, \ldots, b_{r-1}\right\} \quad \text { and } \quad \boldsymbol{u}=\left\{u_{1}, \ldots, u_{k}\right\},
$$

where $\operatorname{deg} a_{i}=\operatorname{deg} \xi u_{j}=(1,1), \operatorname{deg} b_{i}=\operatorname{deg} u_{j}=(1,0)$, while $r$ and $k$ are indicated in each case below, and a variable $\eta$ with $\operatorname{deg} \eta=(2,1)$. 
(I) If $\Sigma=\Sigma_{g, r}^{1}$ then $g+1=r+2 q$, and the $\mathcal{D}$-module structure of $H_{\mathcal{D} / \mathbb{R}}^{*}(\Sigma ; \mathbb{Z}(\bullet))$ is determined by

$$
H_{\mathcal{D} / \mathbb{R}}^{*}(\Sigma ; \mathbb{Z}(\bullet)) \cong \mathcal{D} \cdot \mathbf{1} \oplus \tilde{\mathcal{D}} \otimes \mathbb{Z}(\boldsymbol{a}) \oplus \mathrm{X}_{\mathcal{D}}^{\Sigma}(\boldsymbol{u}, \boldsymbol{b}) \oplus \mathcal{D} \cdot \eta,
$$

with $\boldsymbol{u}=\left\{u_{1}, \ldots, u_{2 q}\right\}$. The ring structure is determined by the relations:

(1) $\left(\lambda \otimes a_{i}\right) \cdot\left(\lambda^{\prime} \otimes a_{j}\right)=\delta_{i j}\left(\lambda \lambda^{\prime} \varepsilon\right) \otimes a_{i}$ for all $\lambda, \lambda^{\prime} \in M$.

(2) $\left(1 \otimes b_{i}\right) \cdot\left(1 \otimes b_{j}\right)=0$.

(3) $\left(\lambda \otimes a_{i}\right) \cdot\left(1 \otimes b_{j}\right)=\delta_{i j} \lambda \eta$.

(4) $x \cdot y=x \cdot \eta=y \cdot \eta=0$ for all $x \in \tilde{\mathcal{D}}(\boldsymbol{a})+\mathrm{X}_{\mathcal{D}}(\boldsymbol{b}), y \in \mathbf{X}_{\mathcal{D}}(\boldsymbol{u})$.

(5) $\left(\lambda \otimes \xi u_{i}\right) \cdot\left(\lambda^{\prime} \otimes \xi u_{j}\right)=0$ for all $i, j$, and $\lambda, \lambda^{\prime} \in M$.

(2) with $\Gamma_{s}^{\Sigma}$ given in Table 5 above,

$$
\begin{cases}\left(\xi^{-k} u_{i}\right) \cdot\left(\xi^{-l} u_{j}\right)=(-1)^{k} \delta_{i+j, 2 q+1} \Gamma_{-k-l}^{\Sigma} & k, l \geq 0, \\ \left(\xi^{-k} u_{i}\right) \cdot\left(\lambda \otimes \xi u_{j}\right)=(-1)^{k} \delta_{i+j, 2 q+1} \lambda \Gamma_{1-k}^{\Sigma} & k \geq 0, \lambda \in M .\end{cases}
$$

(II) If $\Sigma=\Sigma_{g, r}^{0}$ with $1 \leq r \leq g$, then the $\mathcal{D}$-module structure of $H_{\mathcal{D} / \mathbb{R}}^{*}(\Sigma ; \mathbb{Z}(\bullet))$ is determined by

$$
H_{\mathcal{D} / \mathbb{R}}^{*}(\Sigma ; \mathbb{Z}(\bullet)) \cong \mathcal{D} \cdot \mathbf{1} \oplus \tilde{\mathcal{D}} \otimes \mathbb{Z}(\boldsymbol{a}) \oplus \mathrm{X}_{\mathcal{D}}^{\Sigma}(\boldsymbol{u}, \boldsymbol{b}) \oplus \mathcal{D} \cdot \eta,
$$

where we have $\boldsymbol{a}=\left\{a_{1}, \ldots, a_{r-1}\right\}, \underline{b}=\left\{b_{1}, \ldots, b_{r-1}\right\}, \boldsymbol{u}=\left\{u_{1}, \ldots, u_{g+1-r}\right\}$, $\operatorname{deg} u_{i}=\operatorname{deg} b_{i}=(1,0), \xi \boldsymbol{u}=\left\{\xi u_{1}, \ldots, \xi u_{g+1-r}\right\}$ and $\operatorname{deg} a_{i}=\operatorname{deg} \xi u_{j}=(1,1)$. The ring structure is determined by the relations (1)-(5) as in (I) and by

$$
\text { (2) } \begin{cases}\left(\xi^{-k} u_{i}\right) \cdot\left(\xi^{-l} u_{j}\right)=(-1)^{k} \delta_{i j} \Gamma_{-k-l}^{\Sigma} & k \geq 1, l \geq 0, \\ \left(\xi^{-k} u_{i}\right) \cdot\left(\lambda \otimes \xi u_{j}\right)=(-1)^{k} \delta_{i j} \lambda \Gamma_{1-k}^{\Sigma} & k \geq 0, \lambda \in M .\end{cases}
$$

(III) If $\Sigma=\Sigma_{g, 0}^{0}$ with $g=2 q$, then the $\mathcal{D}$-module structure of $H_{\mathcal{D} / \mathbb{R}}^{*}(\Sigma ; \mathbb{Z}(\bullet))$ is given by

$$
H_{\mathcal{D} / \mathbb{R}}^{*}(\Sigma ; \mathbb{Z}(\bullet)) \cong H_{\mathcal{D} / \mathbb{R}}^{*}(B ; \mathbb{Z}(\bullet)) \oplus \mathrm{X}_{\mathcal{D}_{\mathrm{EV}}}^{\Sigma}(\boldsymbol{u}),
$$

where $B$ is the Brauer-Severi curve, $\operatorname{deg} \eta=(2,1), \boldsymbol{u}=\left\{u_{1}, \ldots, u_{2 q}\right\}$ and $\operatorname{deg} u_{i}=(1,0)$. The ring structure is determined by: $H_{\mathcal{D} / \mathbb{R}}^{*}(B ; \mathbb{Z}(\bullet))$ is a subring, the relation

(1) $x \cdot \eta=0$ for all $x \in X_{\mathcal{D}}^{\Sigma}(\boldsymbol{u})$,

and by relations (5) and (\%).

(IV) If $\Sigma=\Sigma_{g, 0}^{0}$ with $g=2 q+1$, then the $\mathcal{D}$-module structure of $H_{\mathcal{D} / \mathbb{R}}^{*}(\Sigma ; \mathbb{Z}(\bullet))$ is given by

$$
H_{\mathcal{D} / \mathbb{R}}^{*}(\Sigma ; \mathbb{Z}(\bullet)) \cong \mathcal{D}_{\mathrm{EV}} / J_{\mathrm{EV}}^{2} \cdot \mathbf{1} \oplus \tilde{\mathcal{D}}_{\mathrm{EV}} / J_{\mathrm{EV}}^{2} \cdot \mathrm{S} \oplus \mathrm{X}_{\mathcal{D}_{\mathrm{EV}}}^{\Sigma}(\boldsymbol{u}, \mathrm{T}) \oplus \mathcal{D}_{\mathrm{EV}} / J_{\mathrm{EV}} \cdot \eta,
$$


where $\boldsymbol{u}=\left\{u_{1}, \ldots, u_{2 q}\right\}, \operatorname{deg} u_{i}=(1,0)$. The ring structure is determined by the relations (1), (2) and (3) in Proposition 5.2, together with (5), (\$) and

(1) $x \cdot \mathrm{T}=x \cdot \eta=0$ for all $x \in \mathrm{X}_{\mathcal{D}_{\mathrm{EV}}}^{\Sigma}(\boldsymbol{u})$.

Proof Case I Define $\mathcal{R}$ as the bigraded $\mathcal{D}$-module

$$
\mathcal{R}:=\mathcal{D} \cdot \mathbf{1} \oplus \widetilde{\mathcal{D}} \otimes \mathbb{Z}\{\boldsymbol{a}\} \oplus \mathrm{X}_{\mathcal{D}}^{\Sigma}(\boldsymbol{u}, \boldsymbol{b}) \oplus \mathcal{D} \cdot \eta
$$

equipped with the $\mathcal{D}$-algebra structure determined by relations (1)-(5) and (\$) in the statement. Denote

$$
\mathcal{R}^{\leq 0}:=\bigoplus_{p \leq 0} \mathcal{R}^{*, p}, \quad \mathcal{R}^{>1}:=\bigoplus_{p>1} \mathcal{R}^{*, p}, \quad H_{\mathcal{D} / \mathbb{R}}^{\leq 0}:=\bigoplus_{p \leq 0} H_{\mathcal{D} / \mathbb{R}}^{*, p},
$$

and observe that $\mathcal{R}^{\leq 0}$ and $\mathcal{R}^{>1}$ are subrings of $\mathcal{R}$, while $H_{\mathcal{D} / \mathbb{R}}^{\leq 0}$ is a subring of $H_{\mathcal{D} / \mathbb{R}}^{*, \bullet}$. Next we introduce a ring homomorphism $F: \mathcal{R}^{*, \bullet} \rightarrow H_{\mathcal{D} / \mathbb{R}}^{*}(\Sigma ; \mathbb{Z}(\bullet))$, defining it separately on $\mathcal{R}^{\leq 0}$ and $\mathcal{R}^{\geq 1}$.

The case $\mathcal{R}^{\leq \mathbf{0}}$ First note that $\mathcal{R}^{\leq 0}$ is isomorphic to $H_{\mathrm{Br}}^{\leq 0}(\Sigma(\mathbb{C}) ; \mathbb{Z})$ which, by definition, coincides with $H_{\mathcal{D} / \mathbb{R}}^{\leq 0}$. Hence, we can define $F$ on $\mathcal{R} \leq 0$ as the isomorphism of Theorem 4.1.

The case $\mathcal{R}^{\geq 1}$ By definition, the elements $a_{i}$ do not belong to $\mathcal{R}$ but can be identified with elements in $H_{\mathrm{Br}}^{1,1}$, using the presentation in Theorem 4.1. We can use the map $b_{\mathbb{R}}$ of Lemma 5.4 and the pairing $\mathrm{m}_{\mathbb{R}}$ of Lemma 5.6 to define $F$ on $\widetilde{\mathcal{D}}\{\boldsymbol{a}\} \cap \mathcal{R}^{\geq 1}=\mathcal{D}_{+} \otimes \mathbb{Z}\{\boldsymbol{a}\}$ by

$$
\digamma\left(\sum_{i} \lambda_{i} \otimes a_{i}\right):=\sum_{i} b_{\mathbb{R}} \mathrm{m}_{\mathbb{R}}\left(\lambda_{i} \otimes a_{i}\right)
$$

see Remark 5.5.

Let $L_{0} \in \operatorname{Pic}_{\mathbb{R}}(\Sigma)=H_{\mathcal{D} / \mathbb{R}}^{2}(\Sigma ; \mathbb{Z}(1))$ be the line bundle associated to the divisor defined by the chosen base point $p_{0} \in \Sigma(\mathbb{R})$. We define $F$ on $\mathcal{D} \cdot \eta \cap \mathcal{R}^{\geq 1}=\mathcal{D}_{0} \cdot \eta$ by sending $\eta$ to $L_{0}$.

Now, it remains to define $F$ on

$$
\mathrm{X}_{\mathcal{D}}^{\Sigma}(\boldsymbol{u}, \boldsymbol{b}) \cap \mathcal{R}^{\geq 1}=\left(\mathcal{D}_{+} \otimes \mathbb{Z}\{\boldsymbol{b}\} \oplus M \otimes \mathbb{Z}\{\boldsymbol{u}\}\right) / \mathscr{F}_{\mathrm{X}^{\Sigma}} \oplus M \otimes \mathbb{Z}\{\xi \boldsymbol{u}\} .
$$

Once again, the elements $\xi u_{i}$ do not belong to $\mathcal{R}$ but can be seen as elements in $H_{\mathrm{Br}}^{1,1}$ and we use the arguments in (45) to define $F$ on $M \otimes \mathbb{Z}\{\xi \boldsymbol{u}\}$ as

$$
F\left(\sum_{i} \lambda_{i} \otimes \xi u_{i}\right):=\sum_{i} b_{\mathbb{R}} \mathrm{m}_{\mathbb{R}}\left(\lambda_{i} \otimes \xi u_{i}\right) .
$$


Finally, since both $\boldsymbol{u}$ and $\boldsymbol{b}$ can be identified with elements in $H_{\mathrm{Br}}^{1,0}=H_{\mathcal{D} / \mathbb{R}}^{1,0}$ we use the multiplication in Deligne cohomology to define

$$
F\left(\lambda \otimes b_{i}\right)=\lambda \cdot b_{i} \quad \text { and } \quad F\left(\lambda \otimes u_{i}\right)=\lambda \cdot u_{i},
$$

respectively.

In what follows, we show that $F$ is an isomorphism and, along the way, we also show that $F$ is a well-defined ring homomorphism.

First we analyze the kernel of $F$. Since $F$ is an isomorphism on $\mathcal{R}^{\leq 0}$, it suffices to study separately the kernels of $F^{1,1}, F^{2,1}$ and $F^{>1}$. On $\mathcal{R}^{1,1}=\mathcal{D}^{1,1} \cdot \mathbf{1} \cong \mathbb{R}^{\times}$the map $F^{1,1}$ is clearly an isomorphism onto $H_{\mathcal{D} / \mathbb{R}}^{1,1}=H^{0}\left(\Sigma ; \mathbb{R}^{\times}\right)$.

Now, an element $w \in \operatorname{ker} \digamma^{2,1}$ can be represented by $w=\boldsymbol{s} \otimes \boldsymbol{u}^{t}+\lambda \otimes \boldsymbol{b}^{t}+k \eta$ with $\lambda \in\left(\mathcal{D}^{1,1}\right)^{r-1}, s \in\left(M^{1,1}\right)^{g-r+1}$ (seen as row matrices) and $k \in \mathbb{Z}$. From $\Psi_{\mathcal{B}} F(w)=0$ we get $k=0$ and $\lambda \in\left(M^{1,1}\right)^{r-1}$.

From (6) and Table 6, we have $\mathbf{f}=-\boldsymbol{\vartheta}_{\boldsymbol{\alpha}} \cdot M+2 \boldsymbol{\vartheta}_{\boldsymbol{\beta}}=(\varphi \boldsymbol{u} \varphi \boldsymbol{b}) \Lambda \subset \operatorname{im}\left(H_{\mathbb{Z}(0)}^{1} \hookrightarrow H_{\mathbb{C}}^{1}\right)^{\mathfrak{S}}$. Hence, (47) gives

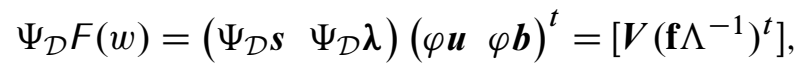

where $\boldsymbol{V}=\left(v_{1}, \ldots, v_{g}\right) \in \mathbb{R}^{g}$ represents $\left(\Psi_{\mathcal{D}} \boldsymbol{s} \Psi_{\mathcal{D}} \lambda\right) \in H_{\mathcal{D} / \mathbb{C}}^{1}(\operatorname{Spec} \mathbb{C} ; \mathbb{Z}(1))^{g}=$ $(\mathbb{C} / \mathbb{Z}(1))^{g}$, and $[c]$ denote the class of an element $c \in H_{\mathbb{C}}^{1}$ in the Jacobian of $\Sigma_{\mathbb{C}}$.

The assumption $\Psi_{\mathcal{D}} F(w)=0$ is equivalent to $V\left(\mathbf{f} \Lambda^{-1}\right)^{t} \in\left\{H_{\mathbb{Z}(1)}^{1}\right\}^{\mathfrak{S}}+\operatorname{span}_{\mathbb{R}}\{\boldsymbol{\omega}\}$, and Proposition 2.4 gives $\mathbf{f}=2 \boldsymbol{i} \vartheta_{\boldsymbol{\alpha}} \mathrm{T}+2 \boldsymbol{\omega}$.

Therefore,

$$
\begin{aligned}
\boldsymbol{V}\left(\mathbf{f} \Lambda^{-1}\right)^{t}=\boldsymbol{n} \cdot\left(2 \pi \boldsymbol{i} \vartheta_{\alpha}\right)^{t}+\boldsymbol{z} \cdot \boldsymbol{\omega}^{t} & =\boldsymbol{n} \cdot\left(\pi \mathbf{f T}^{-1}-2 \pi \boldsymbol{\omega} \mathrm{T}^{-1}\right)^{t}+\boldsymbol{z} \cdot \boldsymbol{\omega}^{t} \\
& =\boldsymbol{n} \cdot \pi \mathrm{T}^{-1} \mathbf{f}^{t}+\left(z-2 \pi \boldsymbol{n} \mathrm{T}^{-1}\right) \cdot \boldsymbol{\omega}^{t}
\end{aligned}
$$

and hence $\boldsymbol{V}=\boldsymbol{n}\left(\pi \Lambda \mathrm{T}^{-1}\right)^{t}$. We use the fact that $\left\{2 \pi \boldsymbol{i} \vartheta_{\boldsymbol{\alpha}}\right\}$ and $\{\mathbf{f}, \boldsymbol{\omega}\}$ are bases for the invariants of $H_{\mathbb{Z}(1)}^{1}$ and $H_{\mathbb{C}}^{1}$, respectively. It follows that $w \in \mathscr{F}_{\pi \Lambda T^{-1}}$.

Note that the arguments above show that $\mathscr{F}_{\pi \Lambda T^{-1}}$ is sent to zero by $F$, and hence in order to complete the proof that $F$ is a well-defined map of $\mathcal{D}$-modules, we are reduced to showing that $F\left(\varepsilon u_{j}\right)=\frac{1}{2} F\left(v_{j}\right)$, where $v_{j}$ is the $j^{\text {th }}$ element in $(\boldsymbol{u} \boldsymbol{b}) \Lambda T^{-1}$. 
However, the arguments used in (48) show that

$$
\begin{aligned}
\Psi_{\mathcal{D}} F(\varepsilon \boldsymbol{u} \varepsilon \boldsymbol{b}) & =[-\pi \boldsymbol{i}(\varphi \boldsymbol{u} \varphi \boldsymbol{b})] \\
& =\left[-\pi \boldsymbol{i}\left(-\vartheta_{\alpha} \cdot M+2 \vartheta_{\beta}\right) \Lambda^{-1}\right] \\
& =\left[\left(\pi \boldsymbol{i} \vartheta_{\alpha}\right) \cdot M \Lambda^{-1}\right]+\left[\left(-2 \pi \boldsymbol{i} \vartheta_{\beta}\right) \Lambda^{-1}\right] \\
& =\left[\frac{1}{2}\left(2 \pi \boldsymbol{i} \vartheta_{\alpha}\right) \cdot M \Lambda^{-1}\right] \\
& =\left[\frac{1}{2}\left(\pi \boldsymbol{e} \mathrm{T}^{-1}\right) \cdot M \Lambda^{-1}\right] \\
& =\left[\frac{1}{2}\left(\pi \boldsymbol{f} \mathrm{T}^{-1}\right) \cdot M \Lambda^{-1}\right] \\
& =\left[\frac{1}{2}(\varphi(\boldsymbol{u}) \varphi(\boldsymbol{b}))\left(\pi \Lambda \mathrm{T}^{-1}\right) \cdot M \Lambda^{-1}\right] \\
& =\left[\left(\frac{1}{2} v_{1}, \ldots, \frac{1}{2} v_{2 q}, 0, \ldots 0\right)\right],
\end{aligned}
$$

since $M \Lambda^{-1}=\left(\begin{array}{cc}I_{2 q} & 0 \\ 0 & 0\end{array}\right)$.

To show that $F^{>1}$ is one-to-one it suffices by Lemma 5.9 to show that $\Psi F$ is one-to-one. A relevant observation at this point is the fact that $\mathscr{F}_{X}$ has only nonzero elements in degree $(2,1)$, implying that an element $x \in \operatorname{ker} \Psi_{\mathcal{B}} F^{>1}$ can be uniquely written as $x=\sum_{i} \lambda_{i} \otimes a_{i}+\sum_{j} \mu_{j} \otimes b_{j}+\sum_{k} m_{k} \otimes u_{k}+\sum_{l} n_{l} \otimes\left(\xi u_{l}\right)$, with $m_{k}, n_{l} \in M$. Since $M=\operatorname{ker}\left\{\Psi_{\mathcal{B}}: \mathcal{D} \rightarrow \mathcal{B}\right\}$, we conclude from Propositions 5.4 and 5.6 that

$$
\begin{aligned}
0=\Psi_{\mathcal{B}} F(x) & =\Psi_{\mathcal{B}} F\left(\sum_{i} \lambda_{i} \otimes a_{i}+\sum_{j} \mu_{j} \otimes b_{j}\right) \\
& =\Psi_{\mathcal{B}}\left(\sum_{i} b_{\mathbb{R}} \mathrm{m}_{\mathbb{R}}\left(\lambda_{i} \otimes a_{i}\right)+\sum_{j} \mu_{j} \cdot b_{j}\right) \\
& =\sum_{i} \Psi_{\mathcal{B}}\left(\lambda_{i}\right) \cdot a_{i}+\sum_{j} \Psi_{\mathcal{B}}\left(\mu_{j}\right) \cdot b_{j} .
\end{aligned}
$$

Theorem 4.1 implies that $\Psi_{\mathcal{B}}\left(\lambda_{i}\right)=\Psi_{\mathcal{B}}\left(\mu_{j}\right)=0$, ie $\lambda_{i}, \mu_{j} \in M$ for all $i, j$, and this fact together with Proposition 5.10 and $\Psi_{\mathcal{D}} F(x)=0$ implies that $x=0$.

Next we show that $F: \mathcal{R}^{>1} \rightarrow H_{\mathcal{D}}^{>1}$ is onto. We start by noting that from the definition of $F$ and Theorem 4.1 it is clear that $\Psi_{\mathcal{B}} F$ is onto $H_{\mathcal{B}}^{>1}$.

Let $a \in H_{\mathcal{D} / \mathbb{R}}^{n, p}$ (with $p>1$ ), set $b=\Psi_{\mathcal{B}}(a)$ and choose $x \in \mathcal{R}$ such that $\Psi_{\mathcal{B}} F(x)=b$. Since $\Psi_{\mathcal{B}}(F(x)-a)=0$ it follows from (12) that there exists $y \in\left\{H_{\mathbb{C}}^{n-1}\right\}^{\mathfrak{S}}$ such that $\delta_{\mathbb{R}}(y)=F(x)-a$. Then $\Psi_{\mathcal{D}} \delta_{\mathbb{R}}(y) \in\left\{H_{\mathcal{D} / \mathbb{C}}^{n, p}\right\}_{0}^{\mathfrak{S}}$ (the component of zero) and it follows from the presentation of $H_{\mathcal{D} / \mathbb{C}}^{*, \bullet}$ in Proposition 5.10 and the definition of $F$ that there is 
$z \in \operatorname{ker} \Psi_{\mathcal{B}} F$ such that $\Psi_{\mathcal{D}} F(z)=\Psi_{\mathcal{D}} \delta_{\mathbb{R}}(y)$. We have

$$
\begin{aligned}
& \Psi_{\mathcal{D}} F(x-z)=\Psi_{\mathcal{D}}\left(\delta_{\mathbb{R}}(y)+a-\delta_{\mathbb{R}}(y)\right)=\Psi_{\mathcal{D}}(a), \\
& \Psi_{\mathcal{B}} F(x-z)=\Psi_{\mathcal{B}} F(x)=\Psi_{\mathcal{B}}(a),
\end{aligned}
$$

hence $F(x-z)=a$. We conclude from Lemma 5.9 that $F: \mathcal{R}^{>1} \rightarrow H_{\mathcal{D} / \mathbb{R}}^{>1}$ is onto.

It remains to show that $F^{2,1}$ is onto. From Theorem 4.1 and the definition of $F$ it suffices to show that $F^{2,1}$ maps onto $\operatorname{ker} \Psi_{\mathcal{B}}^{2,1}$. From (12) we have

$$
\operatorname{ker} \Psi_{\mathcal{B}}^{2,1} \cong\left\{H_{\mathbb{C}}^{1}\right\}^{\mathfrak{S}} / \varphi H_{\mathrm{Br}}^{1,1}+\left\{F^{1} H_{\mathbb{C}}^{1}\right\}^{\mathfrak{S}} \text {. }
$$

The result now follows from the equality $\left\{H_{\mathbb{C}}^{1}\right\}^{\mathfrak{S}}=\operatorname{span}_{\mathbb{R}}\{\varphi \boldsymbol{u}, \varphi \boldsymbol{b}\}+\left\{F^{1} H_{\mathbb{C}}^{1}\right\}^{\mathfrak{S}}$, and the definition of $F$ : for $\lambda \in \mathbb{R}$ it is easy to check that $\lambda \mathrm{E} \cdot u_{i}=\delta_{\mathbb{R}}\left(\lambda \varphi\left(u_{i}\right)\right)$ and $\lambda \mathrm{E} \cdot b_{i}=\lambda_{\mathbb{R}}\left(\lambda \varphi\left(b_{i}\right)\right)$, where $\delta_{\mathbb{R}}$ is the connecting homomorphism in (12).

Putting together the results on the image and kernel of $F$ we conclude that it induces the isomorphism of $\mathcal{D}$-modules

$$
\mathcal{R} / \mathscr{I}_{\mathscr{g} a c}\left(\Lambda \mathrm{T}^{-1}, \boldsymbol{u}, \boldsymbol{b}\right) \rightarrow H_{\mathcal{D} / \mathbb{R}}^{\star, \bullet}
$$

in the statement of the theorem. It is easy to check that $\Psi_{\mathcal{B}} F$ and $\Psi_{\mathcal{D}} F$ are ring homomorphisms (Theorem 4.1 and Proposition 5.10) and hence so is $F$.

Case II The proof of (I) applies because Lemma 5.9 still holds.

Case III Similar to (IV) below.

Case IV The definition of the $\mathcal{D}$-module $\mathcal{R}$ is analogous to that in Case I with the obvious adaptations:

$$
\mathcal{R}:=\mathcal{D}_{\mathrm{EV}} / J_{\mathrm{EV}}^{2} \cdot \mathbf{1} \oplus \widetilde{\mathcal{D}}_{\mathrm{EV}} / J_{\mathrm{EV}}^{2} \otimes \mathbb{Z}\{\mathrm{S}\} \oplus \mathrm{X}_{\mathcal{D}}^{\Sigma}(\boldsymbol{u}, \mathrm{T}) \oplus \mathcal{D}_{\mathrm{EV}} / J_{\mathrm{EV}} \cdot \eta,
$$

where $\boldsymbol{u}=\left(u_{1}, \ldots, u_{g-1}\right)$. The definition of the map $F: \mathcal{R} \rightarrow H_{\mathcal{D} / \mathbb{R}}^{\star, \bullet}$ follows along the same lines as in (I) with the following changes, due to the fact that Lemma 5.9 does not hold.

(i) The injectivity of $F$ on $\mathcal{R}^{>1}$ follows by checking directly on the elements that map onto $\operatorname{ker} \Psi$. Using (12) we then show that $\operatorname{ker} \Psi^{>1}$ is generated by elements of the form $\delta_{\mathbb{R}}\left(\frac{1}{2} \varphi \mathrm{E} \tau^{r} \cdot \mathrm{E}\right) \in H_{\mathcal{D} / \mathbb{R}}^{2,1+2 r}$ and $\delta_{\mathbb{R}}\left(\frac{1}{2} \mathrm{Y} \tau^{r} \cdot \eta\right) \in H_{\mathcal{D} / \mathbb{R}}^{3,1+2 r}$, and it is clear $F$ maps $\frac{1}{2} \mathrm{E} \tau^{r} \cdot \mathrm{E}, \frac{1}{2} \mathrm{Y} \tau^{r} \cdot \eta$ bijectively onto these elements.

(ii) The surjectivity of $F: \mathcal{R}^{>1} \rightarrow H_{\mathcal{D} / \mathbb{R}}^{>1}$ follows from the fact that $\digamma$ maps onto $\operatorname{ker} \Psi_{\mathcal{B}}$ as noted in (i).

This concludes the proof. 
Acknowledgments The first author was partially supported by FCT (Portugal). The second author would like to thank the hospitality of the Instituto Superior Técnico during the elaboration of parts of this work.

\section{References}

[1] GE Bredon, Equivariant cohomology theories, Lecture Notes in Math., Springer (1967) MR0214062

[2] S R Costenoble, S Waner, Equivariant simple Poincaré duality, Michigan Math. J. 40 (1993) 577-604 MR1236180

[3] D Dugger, Bigraded cohomology of $\mathbb{Z} / 2$-equivariant Grassmannians arXiv: 1209.5067

[4] H Esnault, E Viehweg, Deligne-Beilinson cohomology, from: "Beب̌linson's conjectures on special values of $L$-functions", (M Rapoport, N Schappacher, P Schneider, editors), Perspect. Math. 4, Academic Press, Boston (1988) 43-91 MR944991

[5] B H Gross, J Harris, Real algebraic curves, Ann. Sci. École Norm. Sup. 14 (1981) 157-182 MR631748

[6] G Lewis, JP May, J McClure, Ordinary $\mathrm{RO}(G)$-graded cohomology, Bull. Amer. Math. Soc. 4 (1981) 208-212 MR598689

[7] L G Lewis, Jr, The $\mathrm{RO}(G)$-graded equivariant ordinary cohomology of complex projective spaces with linear $\mathbf{Z} / p$ actions, from: "Algebraic topology and transformation groups", (T tom Dieck, editor), Lecture Notes in Math. 1361, Springer (1988) 53-122 MR979507

[8] C Pedrini, C Weibel, Invariants of real curves, Rend. Sem. Mat. Univ. Politec. Torino 49 (1991) 139-173 MR1219265

[9] C Pedrini, C Weibel, The higher K-theory of real curves, $K$-Theory 27 (2002) 1-31 MR1936583

[10] P F dos Santos, P Lima-Filho, Quaternionic algebraic cycles and reality, Trans. Amer. Math. Soc. 356 (2004) 4701-4736 MR2084395

[11] P F dos Santos, P Lima-Filho, Bigraded equivariant cohomology of real quadrics, Adv. Math. 221 (2009) 1247-1280 MR2518638

[12] P F dos Santos, P Lima-Filho, Integral Deligne cohomology for real varieties, Math. Ann. 350 (2011) 973-1022 MR2818720

[13] R Silhol, Compactifications of moduli spaces in real algebraic geometry, Invent. Math. 107 (1992) 151-202 MR1135469

[14] R Silhol, Moduli problems in real algebraic geometry, from: "Real algebraic geometry", (M Coste, L Mahé, M-F Roy, editors), Lecture Notes in Math. 1524, Springer (1992) 110-119 MR1226244 
Departamento de Matemática, Instituto Superior Técnico, Universidade de Lisboa Avenida Rovisco Pais, 1049-001 Lisboa, Portugal

Department of Mathematics, Texas A\&M University

Mailstop 3368, College Station, TX 77843, USA

pedro.f.santos@tecnico.ulisboa.pt, plfilho@math.tamu.edu

www.math.tecnico.ulisboa.pt/ pedfs, www.math.tamu.edu/ plfilho

Received: 2 August 2013 Revised: 14 January 2014 